\title{
ALASKA MARINE MAMMAL TISSUE ARCHIVAL PROJECT: SPECIMEN INVENTORY
}

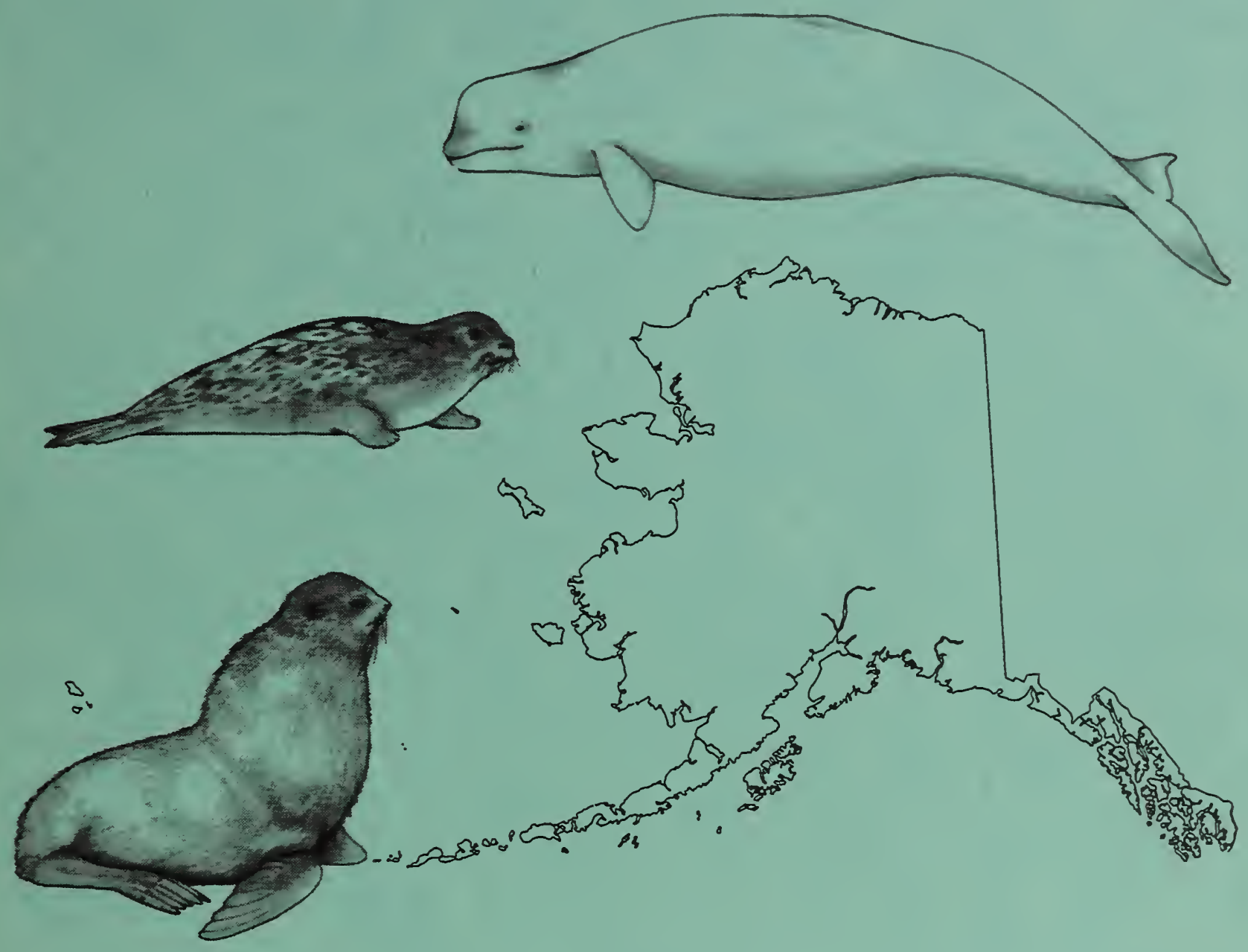

Barbara J. Koster and Stephen A. Wise

U.S. DEPARTMENT OF COMMERCE

National Institute of Standards and Technology Chemical Science and Technology Laboratory Gaithersburg, Maryland 20899
QC

100

.456

1994

N0. 5462
Paul R. Becker

U.S. DEPARTMENT OF COMMERCE

National Oceanic and Atmospheric Administration National Marine Fisheries Service

Office of Protected Resources

Silver Spring, Maryland 20910

U.S. DEPARTMENT OF COMMERCE

Ronald H. Brown, Secretary

NATIONAL INSTITUTE OF STANDARDS AND TECHNOLOGY

Arati Prabhakar, Director 

NISTIR 5462

\section{ALASKA MARINE MAMMAL TISSUE ARCHIVAL PROJECT: SPECIMEN INVENTORY}

Barbara J. Koster and Stephen A. Wise

U.S. DEPARTMENT OF COMMERCE

National Institute of Standards and Technology

Chemical Science and Technology Laboratory

Gaithersburg, Maryland 20899

\section{Paul R. Becker}

U.S. DEPARTMENT OF COMMERCE

National Oceanic and Atmospheric Administration

National Marine Fisheries Service

Office of Protected Resources

Silver Spring, Maryland 20910 .

November 1994

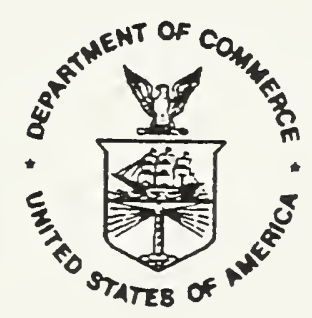

U.S. DEPARTMENT OF COMMERCE

Ronald Brown, Secretary

NATIONAL INSTITUTE OF STANDARDS

AND TECHNOLOGY

Arati Prabakhar 



\begin{abstract}
The Alaska Marine Mammal Tissue Archival Project (AMMTAP) was initiated in 1987 to aid in the monitoring of contaminants in the Alaskan environment. Standardized sampling protocols for the collection of marine mammal tissue were developed to ensure representative and non-contaminated specimens. The tissues samples are then archived in the National Biomonitoring Specimen Bank (NBSB) at the National Institute of Standards and Technology (NIST). In the NBSB, the tissues are stored in vapor phase liquid nitrogen freezers until required for analysis. Organic and inorganic analyses have been performed on approximately $75 \%$ of the animals to establish baseline data so that comparison can be made with other data and future analyses. This document contains the current inventory of the AMMTAP archive. Included in the inventory is information such as: species, location collected, morphometrics, tissues collected, and dispositions of the tissue, i.e., archived or homogenized for analysis. This document contains the records for the collection of tissue from 121 animals sampled between July 87 - July 94 . The collections are continuing with the support of the National Oceanic and Atmospheric Administration (NOAA) and the National Biological Survey (NBS).
\end{abstract}

Key Words: Alaska; tissue archival; marine mammals; specimen banking; inventory 



\section{PREFACE}

In 1987, the Minerals Management Service (MMS) provided funds to the Ocean Assessments Division (OAD) Alaska Office, National Oceanic and Atmospheric Administration (NOAA), to establish and conduct a project for the collection and long-term storage of tissues from Alaska marine mammals for future chemical analysis. Chemical analysis of marine mammal tissues can be particularly useful in determining whether bioaccumulation of contaminants and potential biological effects associated with human industrial activities, including offshore petroleum and mineral extraction, is occurring in marine food webs. This project, the Alaska Marine Mammal Tissue Archival Project (AMMTAP), has been conducted as a cooperative effort between OAD and the National Institute of Standards and Technology (NIST) through the latter's National Biomonitoring Specimen Bank (NBSB). The collection and archival of such tissue samples over a period of several years will provide a resource that can be used to determine baseline contaminant levels against which future contaminant measurements can be compared.

With the closure of the OAD Alaska Office in early 1992, the management of the AMMTAP was transferred to the National Marine Fisheries Service's Office of Protected Resources. The Office of Protected Resources manages the National Marine Mammal Tissue Bank (NMMTB) as a component of the Marine Mammal Health and Stranding Response Program. This program was established in 1992 by P.L. 102-587 to facilitate the collection and dissemination of data on the health of marine mammals and health trends in marine mammal populations in the wild; to correlate these trends with available data on physical, chemical, and biological environmental parameters; and to coordinate effective responses to unusual marine mammal mortalities. The NMMTB is similar to the AMMTAP, using basically the same collection/archival protocols and the same tissue bank (NBSB), but being directed toward marine mammals of the United States outside Alaska. The AMMTAP is continuing in 1994, under the sponsorship of the National Biological Survey.

This volume represents an inventory of all specimens collected by the AMMTAP and archived in the NBSB and provides a record of data collected on each animal sampled by the Project. Anyone wishing to obtain aliquots of any of these samples for research should refer to the tissue access policy as stated on page 8 of Alaska Marine Mammal Tissue Archival Project: Revised Collection Protocol, NISTIR 4529, NIST, Gaithersburg, Maryland, 1991. 


\section{CONTENTS}

\section{FIGURES:}

1. Geographic locations of sample collections

\section{TABLES:}

1. Marine mammals sampled in the Arctic Ocean

2. Marine mammals sampled in the Gulf of Alaska

3. Marine mammals sampled in the Bering Sea

4. AMMTAP sample inventory format

5. Acronyms used in AMMTAP sample inventory

\section{SAMPLE INVENTORY:}

Ringed Seal (Phoca hispida)

Harbor Seal (Phoca vitulina)

Spotted Seal (Phoca largha)

Bearded Seal (Erignathus barbatus)

Steller Sea Lion (Eumatopias jubatus)

Northern Fur Seal (Callorhinus ursinus)

Pacific Walrus (Odobenus rosmarus rosmarus)

46

Beluga Whale (Delphinapterus leucas) 


\section{ACKNOWLEDGMENTS}

This specimen inventory reflects the cooperation, collaboration, and support of a large number of individuals and organizations within and outside Alaska. Most prominent among which have been:

North Slope Borough Department of Wildlife Management, North Slope Borough Fish and Game Management Committee, Kawerak, Inc., Eskimo Walrus Commission, Alaska Eskimo Whaling Commission, TDX Corporation, Aleut Community of St. Paul, Point Hope Whalers, Point Lay Village, English Bay Village Council, New Chenega Bay Village Council, Tatitlek Village Council, Alaska and Inuvialuit Beluga Whale Committee, Alaska Department of Fish and Game, National Marine Fisheries Service, U.S. Department of Fish and Wildlife, Minerals Management Service, and Marine Mammal Commission.

Jon Nickles and Dana Seagars of the USFWS Marine Mammals Management Office provided a letter of authority to collect tissue samples from subsistence-harvested walrus; all other animals were sampled under the authority of NMFS Permit Nos. 635 and 839.

This study was funded by the NBS through an Interagency Agreement with NOAA. Funding support was provided by MMS Environmental Studies Program. 

The current inventory of samples maintained by the National Biomonitoring Specimen Bank for the AMMTAP is presented in the following pages. Figure 1 presents the geographic locations of the species sampled by the AMMTAP. The listings of the tissue samples archived for each of these geographic locations are presented in Tables 1-3.

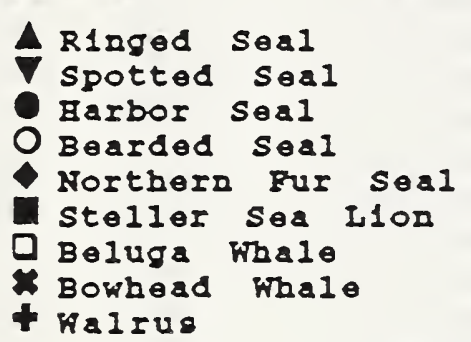

\section{Arctic Ocean}

Chukchi Sea

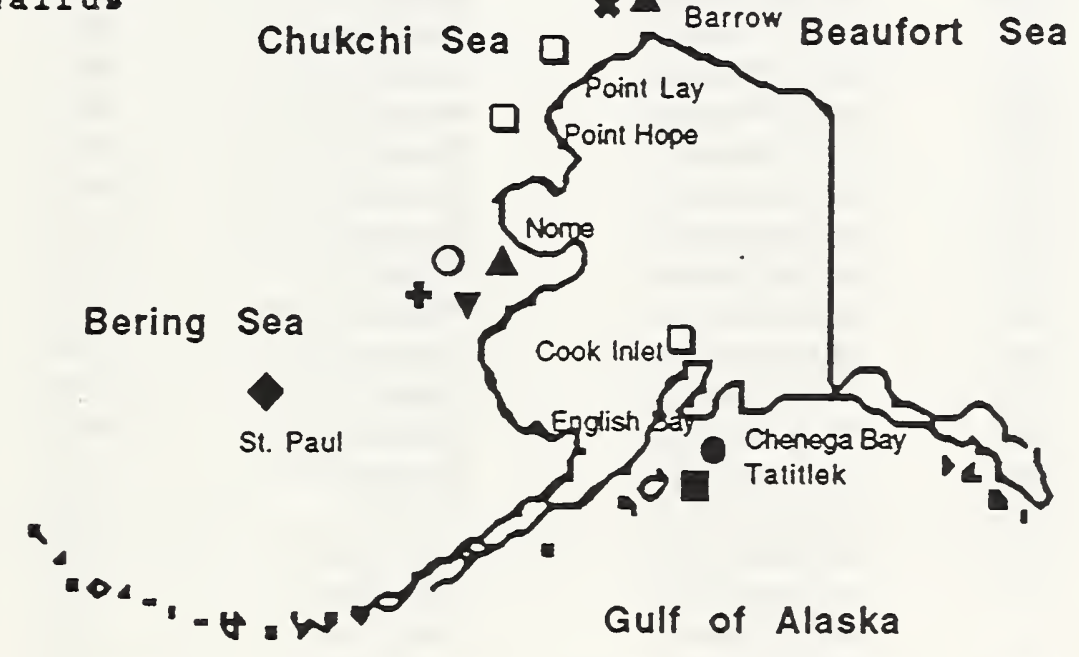

Figure 1. Geographic locations of sample collections 
Table 1. Marine Mammals sampled in the Arctic Ocean (O - samples archived; X - subsample B homogenized and divided into aliquots for analyses)

\begin{tabular}{|c|c|c|c|c|c|c|c|c|}
\hline \multirow[b]{2}{*}{ Species } & \multirow[b]{2}{*}{ Sex } & \multirow[b]{2}{*}{ Individual ID } & \multirow[b]{2}{*}{ Location } & \multirow[b]{2}{*}{ Date } & \multicolumn{4}{|c|}{ Tissue $^{\mathrm{a}}$} \\
\hline & & & & & L & $\mathrm{K}$ & B & $\mathbf{M}$ \\
\hline Ringed Seal & $\mathbf{M}$ & 692-RGSL-001 & Barrow & $7 / 88$ & 0 & 0 & 0 & \\
\hline Ringed Seal & $\mathrm{F}$ & 692-RGSL-002 & Barrow & $7 / 88$ & 0 & 0 & 0 & \\
\hline Ringed Seal & $\mathbf{M}$ & 692-RGSL-003 & Barrow & $7 / 88$ & 0 & 0 & 0 & \\
\hline Ringed Seal & $\mathbf{M}$ & 692-RGSL-004 & Barrow & $7 / 88$ & $\mathrm{X}$ & $\mathrm{X}$ & $\mathrm{X}$ & \\
\hline Ringed Seal & $F$ & 692-RGSL-005 & Barrow & $7 / 88$ & 0 & 0 & 0 & \\
\hline Ringed Seal & $\mathrm{F}$ & 692-RGSL-006 & Barrow & $7 / 88$ & 0 & 0 & 0 & \\
\hline Ringed Seal & $\mathbf{M}$ & 692-RGSL-007 & Barrow & $7 / 88$ & 0 & 0 & 0 & \\
\hline Ringed Seal & $\mathbf{M}$ & 692-RGSL-008 & Barrow & $7 / 88$ & $\mathrm{X}$ & $\mathrm{X}$ & $\mathrm{X}$ & \\
\hline Ringed Seal & $\mathbf{M}$ & 692-RGSL-009 & Barrow & $7 / 88$ & 0 & 0 & 0 & \\
\hline Ringed Seal & $\mathbf{F}$ & 692-RGSL-010 & Barrow & $7 / 88$ & 0 & 0 & 0 & \\
\hline Ringed Seal & $\mathbf{M}$ & 692-RGSL-019 & Barrow & $7 / 91$ & 0 & 0 & 0 & \\
\hline Ringed Seal & $\mathbf{M}$ & 692-RGSL-020 & Barrow & $7 / 91$ & 0 & 0 & 0 & \\
\hline Ringed Seal & $\mathbf{M}$ & 692-RGSL-021 & Barrow & $7 / 91$ & 0 & 0 & 0 & \\
\hline Ringed Seal & $\mathbf{M}$ & 692-RGSL-022 & Barrow & $7 / 91$ & 0 & 0 & 0 & \\
\hline Ringed Seal & $F$ & 692-RGSL-023 & Barrow & $7 / 91$ & 0 & 0 & 0 & \\
\hline Ringed Seal & $\mathbf{M}$ & 692-RGSL-024 & Barrow & $7 / 91$ & 0 & 0 & 0 & \\
\hline Ringed Seal & $\mathrm{F}$ & 692-RGSL-025 & Barrow & $7 / 91$ & 0 & 0 & 0 & \\
\hline Ringed Seal & $\mathbf{M}$ & 692-RGSL-026 & Barrow & $7 / 91$ & 0 & 0 & 0 & \\
\hline Ringed Seal & $\mathbf{M}$ & 692-RGSL-027 & Barrow & $7 / 91$ & 0 & 0 & 0 & \\
\hline Ringed Seal & $\mathrm{F}$ & 692-RGSL-028 & Barrow & $7 / 91$ & 0 & 0 & 0 & \\
\hline Ringed Seal & $\mathbf{M}$ & 692-RGSL-037 & Barrow & $7 / 94$ & 0 & 0 & 0 & \\
\hline Ringed Seal & $\mathbf{M}$ & 692-RGSL-038 & Barrow & $7 / 94$ & 0 & 0 & 0 & \\
\hline Ringed Seal & $\mathrm{F}$ & 692-RGSL-039 & Barrow & $7 / 94$ & $\mathrm{O}$ & $\mathrm{O}$ & 0 & \\
\hline Ringed Seal & $\mathbf{M}$ & 692-RGSL-040 & Barrow & $7 / 94$ & 0 & 0 & 0 & \\
\hline Ringed Seal & $\mathbf{M}$ & 692-RGSL-041 & Barrow & $7 / 94$ & 0 & 0 & 0 & \\
\hline Ringed Seal & $\mathbf{M}$ & 692-RGSL-042 & Barrow & $7 / 94$ & $\mathrm{O}$ & $\mathrm{O}$ & $\mathrm{O}$ & \\
\hline Bearded Seal & $\mathbf{M}$ & 692-BDSL-002 & Barrow & $7 / 89$ & 0 & 0 & 0 & \\
\hline Bearded Seal & $\mathbf{M}$ & 692-BDSL-003 & Barrow & $7 / 89$ & 0 & & 0 & \\
\hline Beluga Whale & $\mathrm{F}$ & 692-BLKA-001 & Point Hope & $5 / 89$ & $\mathrm{X}$ & 0 & $\mathrm{X}$ & \\
\hline Beluga Whale & F & 692-BLKA-002 & Point Hope & $5 / 89$ & $\mathrm{X}$ & 0 & $\mathrm{X}$ & \\
\hline Beluga Whale & $\mathrm{F}$ & 692-BLKA-003 & Point Hope & $5 / 89$ & $\mathrm{X}$ & 0 & & \\
\hline Beluga Whale & $\mathbf{M}$ & 692-BLKA-004 & Point Hope & $5 / 89$ & $\mathrm{X}$ & & & \\
\hline Beluga Whale & $\mathrm{F}$ & 692-BLKA-005 & Point Lay & $7 / 90$ & $\mathrm{X}$ & 0 & $\mathrm{X}$ & \\
\hline Beluga Whale & $\mathbf{M}$ & 692-BLKA-006 & Point Lay & $7 / 90$ & $\mathrm{X}$ & 0 & $\mathrm{X}$ & \\
\hline Beluga Whale & $F$ & 692-BLKA-007 & Point Lay & $7 / 90$ & $\mathrm{X}$ & 0 & $\mathrm{X}$ & \\
\hline Beluga Whale & $\mathbf{M}$ & 692-BLKA-008 & Point Lay & $7 / 90$ & $\mathrm{X}$ & 0 & $\mathrm{X}$ & \\
\hline Beluga Whale & $\mathbf{M}$ & 692-BLKA-009 & Point Lay & $7 / 90$ & $\mathrm{X}$ & 0 & $\mathrm{X}$ & \\
\hline Beluga Whale & $\mathbf{M}$ & 692-BLKA-010 & Point Lay & $7 / 90$ & $\mathrm{X}$ & 0 & $\mathrm{X}$ & \\
\hline Beluga Whale & $\mathbf{M}$ & 692-BLKA-011 & Point Lay & $7 / 90$ & $x$ & 0 & $\mathrm{X}$ & \\
\hline Beluga Whale & $\mathrm{F}$ & 692-BLKA-012 & Point Lay & $7 / 90$ & $\mathrm{X}$ & 0 & $\mathrm{X}$ & \\
\hline Beluga Whale & $\mathbf{M}$ & 692-BLKA-013 & Point Lay & $7 / 90$ & $\mathrm{X}$ & 0 & $\mathrm{X}$ & \\
\hline
\end{tabular}

Tissue

$$
\begin{aligned}
& \text { L - Liver } \\
& \text { K - Kidney } \\
& \text { B - Blubber } \\
& \text { M - Muscle }
\end{aligned}
$$


Table 1. (continued)

\begin{tabular}{|c|c|c|c|c|c|c|c|c|}
\hline \multirow[b]{2}{*}{ Species } & \multirow[b]{2}{*}{ Sex } & \multirow[b]{2}{*}{ Individual ID } & \multirow[b]{2}{*}{ Location } & \multirow[b]{2}{*}{ Date } & \multicolumn{4}{|c|}{ Tissue $\mathrm{a}^{\mathrm{a}}$} \\
\hline & & & & & $\mathrm{L}$ & K & B & $\mathbf{M}$ \\
\hline Beluga Whale & $\mathrm{F}$ & 692-BLKA-014 & Point Lay & $7 / 90$ & $\mathrm{X}$ & 0 & $\mathrm{X}$ & \\
\hline Bowhead Whale & $\mathrm{F}$ & 692-BWHD-001 & Barrow & $5 / 92$ & $\mathrm{X}$ & 0 & 0 & \\
\hline Bowhead Whale & $\mathrm{F}$ & 692-BWHD-002 & Barrow & $5 / 92$ & & 0 & $\mathrm{o}$ & \\
\hline Bowhead Whale & $\mathrm{F}$ & 692-BWHD-003 & Barrow & 9/92 & 0 & & 0 & \\
\hline Bowhead Whale & $\mathbf{M}$ & 692-BWHD-004 & Barrow & $9 / 92$ & 0 & & 0 & \\
\hline Bowhead Whale & $F$ & 692-BWHD-005 & Barrow & $9 / 92$ & 0 & & 0 & \\
\hline Bowhead Whale & $\mathrm{F}$ & 692-BWHD-006 & Barrow & 9/92 & $\mathrm{X}$ & & 0 & \\
\hline Bowhead Whale & $\mathbf{M}$ & 692-BWHD-007 & Barrow & 9/92 & $\mathrm{X}$ & & $\mathrm{X}$ & \\
\hline Bowhead Whale & $\mathbf{M}$ & 692-BWHD-008 & Barrow & 9/92 & 0 & & 0 & \\
\hline Bowhead Whale & $\mathbf{M}$ & 692-BWHD-009 & Barrow & 9/92 & 0 & & $\mathrm{O}$ & \\
\hline Bowhead Whale & $\mathrm{F}$ & 692-BWHD-010 & Barrow & $10 / 92$ & 0 & & 0 & \\
\hline Bowhead Whale & $\mathbf{M}$ & 692-BWHD-011 & Barrow & $10 / 92$ & 0 & & 0 & \\
\hline Bowhead Whale & $\mathrm{F}$ & 692-BWHD-012 & Barrow & $4 / 93$ & 0 & & $\mathrm{o}$ & \\
\hline Bowhead Whale & F & 692-BWHD-013 & Barrow & $5 / 93$ & 0 & & 0 & \\
\hline Bowhead Whale & $\mathrm{F}$ & 692-BWHD-014 & Barrow & $5 / 93$ & 0 & & 0 & \\
\hline Bowhead Whale & F & 692-BWHD-015 & Barrow & $5 / 93$ & 0 & & 0 & \\
\hline Bowhead Whale & $\mathrm{F}$ & 692-BWHD-016 & Barrow & $5 / 93$ & 0 & & 0 & \\
\hline Bowhead Whale & $F$ & 692-BWHD-017 & Barrow & $5 / 93$ & 0 & & 0 & \\
\hline Bowhead Whale & $\mathbf{M}$ & 692-BWHD-020 & Barrow & $10 / 93$ & 0 & & 0 & \\
\hline Bowhead Whale & $\mathbf{M}$ & 692-BWHD-021 & Barrow & $10 / 93$ & 0 & & 0 & \\
\hline Bowhead Whale & $\mathbf{M}$ & 692-BWHD-022 & Barrow & $10 / 93$ & & & 0 & \\
\hline Bowhead Whale & $\mathbf{M}$ & 692-BWHD-024 & Barrow & $5 / 94$ & 0 & & 0 & \\
\hline Bowhead Whale & $\mathbf{M}$ & 692-BWHD-025 & Barrow & $5 / 94$ & 0 & & 0 & \\
\hline Bowhead Whale & $\mathbf{M}$ & 692-BWHD-026 & Barrow & $5 / 94$ & & & 0 & \\
\hline Bowhead Whale & $M$ & 692-BWHD-027 & Barrow & $5 / 94$ & 0 & & 0 & \\
\hline Bowhead Whale & $\mathbf{M}$ & 692-BWHD-028 & Barrow & $5 / 94$ & 0 & & $\mathrm{O}$ & \\
\hline Bowhead Whale & $\mathbf{M}$ & 692-BWHD-029 & Barrow & $5 / 94$ & & & 0 & \\
\hline
\end{tabular}

Tissue ${ }^{a}$

L - Liver

K - Kidney

B - Blubber

M - Muscle 
Table 2. Marine mammals sampled in the Gulf of Alaska (O - samples archived; X - subsample B homogenized and divided into aliquots for analyses)

\begin{tabular}{|c|c|c|c|c|c|c|}
\hline Species & Sex & Individual ID & Location & Date & & issue ${ }^{a}$ \\
\hline Harbor Seal & M & 692-HBSL-001 & Prince William Sound & $3 / 90$ & 00 & 0 \\
\hline Harbor Seal & $\mathbf{F}$ & 692-HBSL-002 & Prince William Sound & $4 / 90$ & 00 & 0 \\
\hline Harbor Seal & $\mathbf{M}$ & 692-HBSL-003 & Prince William Sound & $4 / 90$ & 00 & 0 \\
\hline Harbor Seal & $\mathrm{F}$ & 692-HBSL-004 & Anchorage, AK & $6 / 94$ & 00 & 0 \\
\hline Stellar Sea Lion & $\mathrm{F}$ & 692-STSL-001 & Cook Inlet & $3 / 90$ & 00 & 0 \\
\hline Beluga Whale & $\mathbf{M}$ & 692-BLKA-015 & Cook Inlet & $10 / 92$ & $\mathrm{x} 0$ & 0 \\
\hline Beluga Whale & $\mathbf{M}$ & 692-BLKA-016 & Cook Inlet & 7/94 & & 0 \\
\hline Beluga Whale & $\mathrm{F}$ & 692-BLKA-017 & Cook Inlet & $7 / 94$ & & 0 \\
\hline Beluga Whale & $\mathbf{M}$ & 692-BLKA-018 & Cook Inlet & $7 / 94$ & & 0 \\
\hline
\end{tabular}

Tissue

L - Liver

K - Kidney

B - Blubber

M - Muscle 
Table 3. Marine mammals sampled in the Bering Sea ( $\mathrm{O}$ - samples archived; $\mathrm{X}$ - subsample homogenized and divided into aliquots for analyses)

\begin{tabular}{|c|c|c|c|c|c|c|c|c|}
\hline \multirow[b]{2}{*}{ Species } & \multirow[b]{2}{*}{ Sex } & \multirow[b]{2}{*}{ Individual ID } & \multirow[b]{2}{*}{ Location } & \multirow[b]{2}{*}{ Date } & \multicolumn{4}{|c|}{ Tissue $^{a}$} \\
\hline & & & & & $\mathrm{L}$ & K & B & M \\
\hline Ringed Seal & $\mathbf{M}$ & 692-RGSL-011 & Nome & $5 / 89$ & $\mathrm{X}$ & 0 & $\mathrm{X}$ & \\
\hline Ringed Seal & $F$ & 692-RGSL-012 & Nome & $5 / 89$ & $\mathrm{X}$ & 0 & 0 & \\
\hline Ringed Seal & $\mathbf{M}$ & 692-RGSL-013 & Nome & $5 / 89$ & $\mathrm{X}$ & 0 & $\mathrm{X}$ & \\
\hline Ringed Seal & $\mathbf{M}$ & 692-RGSL-014 & Nome & $5 / 89$ & $\mathrm{X}$ & 0 & 0 & \\
\hline Ringed Seal & $\mathrm{F}$ & 692-RGSL-015 & Nome & $5 / 89$ & $\mathrm{X}$ & 0 & 0 & \\
\hline Ringed Seal & $\mathbf{M}$ & 692-RGSL-016 & Nome & $5 / 91$ & $\mathrm{X}$ & 0 & 0 & \\
\hline Ringed Seal & $F$ & 692-RGSL-017 & Nome & $5 / 91$ & $\mathrm{X}$ & 0 & 0 & \\
\hline Ringed Seal & $F$ & 692-RGSL-018 & Nome & $5 / 91$ & $\mathrm{X}$ & 0 & 0 & \\
\hline Ringed Seal & $\mathbf{M}$ & 692-RGSL-029 & Nome & $5 / 93$ & $\mathrm{X}$ & 0 & 0 & \\
\hline Ringed Seal & $\mathbf{M}$ & 692-RGSL-030 & Nome & $5 / 93$ & $\mathrm{X}$ & 0 & 0 & \\
\hline Ringed Seal & $\mathbf{M}$ & 692-RGSL-031 & Nome & $5 / 93$ & $\mathrm{X}$ & 0 & 0 & \\
\hline Ringed Seal & $\mathbf{M}$ & 692-RGSL-032 & Nome & $6 / 93$ & $\mathrm{X}$ & 0 & 0 & \\
\hline Ringed Seal & $\mathbf{M}$ & 692-RGSL-033 & Nome & $5 / 94$ & 0 & 0 & 0 & \\
\hline Ringed Seal & $\mathbf{M}$ & 692-RGSL-034 & Nome & $5 / 94$ & 0 & 0 & 0 & \\
\hline Ringed Seal & $F$ & 692-RGSL-035 & Nome & $5 / 94$ & 0 & 0 & 0 & \\
\hline Ringed Seal & $\mathrm{F}$ & 692-RGSL-036 & Nome & $5 / 94$ & 0 & 0 & 0 & \\
\hline Spotted Seal & $\mathrm{F}$ & 692-SPSL-001 & Nome & $5 / 91$ & $\mathrm{X}$ & 0 & 0 & \\
\hline Bearded Seal & $\mathbf{M}$ & 692-BDSL-001 & Nome & $5 / 89$ & $\mathrm{X}$ & 0 & 0 & \\
\hline Bearded Seal & $\mathbf{M}$ & 692-BDSL-004 & Nome & $5 / 93$ & $\mathrm{X}$ & 0 & 0 & \\
\hline Bearded Seal & $\mathbf{M}$ & 692-BDSL-005 & Nome & $6 / 93$ & $\mathrm{X}$ & 0 & 0 & \\
\hline Bearded Seal & $\mathbf{M}$ & 692-BDSL-006 & Nome & $5 / 94$ & 0 & 0 & 0 & \\
\hline Bearded Seal & $\mathbf{M}$ & 692-BDSL-007 & Nome & $5 / 94$ & 0 & 0 & 0 & \\
\hline Bearded Seal & $\mathbf{M}$ & 692-BDSL-008 & Nome & $5 / 94$ & 0 & 0 & 0 & \\
\hline Bearded Seal & $F$ & 692-BDSL-009 & Nome & $5 / 94$ & 0 & 0 & 0 & \\
\hline Northern Fur Seal & $\mathbf{M}$ & 692-FRSL-001 & St. Paul & $7 / 87$ & 0 & 0 & 0 & 0 \\
\hline Northern Fur Seal & $\mathbf{M}$ & 692-FRSL-002 & St. Paul & $7 / 87$ & 0 & 0 & 0 & 0 \\
\hline Northern Fur Seal & $\mathbf{M}$ & 692-FRSL-003 & St. Paul & $7 / 87$ & 0 & 0 & 0 & 0 \\
\hline Northern Fur Seal & $\mathbf{M}$ & 692-FRSL-004 & St. Paul & $7 / 87$ & $\mathrm{X}$ & $\mathrm{x}$ & $\mathrm{X}$ & $\mathrm{x}$ \\
\hline Northern Fur Seal & $\mathbf{M}$ & 692-FRSL-005 & St. Paul & $7 / 87$ & $\mathrm{X}$ & $\mathrm{X}$ & $\mathrm{X}$ & $\mathrm{x}$ \\
\hline Northern Fur Seal & $\mathbf{M}$ & 692-FRSL-006 & St. Paul & $7 / 90$ & 0 & 0 & 0 & \\
\hline Northern Fur Seal & $\mathbf{M}$ & 692-FRSL-007 & St. Paul & $7 / 90$ & $\mathrm{X}$ & 0 & 0 & \\
\hline Northern Fur Seal & $\mathbf{M}$ & 692-FRSL-008 & St. Paul & $7 / 90$ & 0 & 0 & 0 & \\
\hline Northern Fur Seal & $\mathbf{M}$ & 692-FRSL-009 & St. Paul & $7 / 90$ & 0 & 0 & 0 & \\
\hline Northern Fur Seal & $\mathbf{M}$ & 692-FRSL-010 & St. Paul & $7 / 90$ & $\mathrm{X}$ & 0 & 0 & \\
\hline Northern Fur Seal & $\mathbf{M}$ & 692-FRSL-011 & St. Paul & $7 / 90$ & $\mathrm{X}$ & 0 & 0 & \\
\hline Northern Fur Seal & $\mathbf{M}$ & 692-FRSL-012 & St. Paul & $7 / 90$ & 0 & 0 & 0 & \\
\hline Northern Fur Seal & $\mathbf{M}$ & 692-FRSL-013 & St. Paul & $7 / 90$ & 0 & 0 & 0 & \\
\hline Northern Fur Seal & $\mathbf{M}$ & 692-FRSL-014 & St. Paul & $7 / 90$ & 0 & 0 & 0 & \\
\hline Northern Fur Seal & $\mathbf{M}$ & 692-FRSL-015 & St. Paul & $7 / 90$ & 0 & 0 & 0 & \\
\hline
\end{tabular}

Tissue ${ }^{a}$

$$
\begin{aligned}
& \text { L - Liver } \\
& \text { K - Kidney } \\
& \text { B - Blubber } \\
& \text { M - Muscle }
\end{aligned}
$$


Table 3. (continued)

\begin{tabular}{|c|c|c|c|c|c|c|c|c|}
\hline \multirow[b]{2}{*}{ Species } & \multirow[b]{2}{*}{ Sex } & \multirow[b]{2}{*}{ Individual ID } & \multirow[b]{2}{*}{ Location } & \multirow[b]{2}{*}{ Date } & \multicolumn{4}{|c|}{ Tissue } \\
\hline & & & & & $\mathbf{L}$ & $\mathbf{K}$ & B & $\mathbf{M}$ \\
\hline Pacific Walrus & $\mathbf{M}$ & 692-WLRS-001 & Nome & $6 / 93$ & 0 & 0 & $\mathrm{O}$ & \\
\hline Pacific Walrus & $\mathbf{M}$ & 692-WLLRS-002 & Nome & $6 / 93$ & 0 & 0 & $\mathrm{O}$ & \\
\hline Pacific Walrus & M & 692-WLRS-003 & Nome & $5 / 94$ & 0 & 0 & 0 & \\
\hline Pacific Walrus & $\mathbf{M}$ & 692-WLRS-004 & Nome & $5 / 94$ & 0 & 0 & 0 & \\
\hline Pacific Walrus & $\mathbf{M}$ & 692-WLRS-005 & Nome & $5 / 94$ & $\mathrm{O}$ & 0 & 0 & \\
\hline
\end{tabular}

Tissue ${ }^{a}$

L - Liver

K - Kidney

B - Blubber

M - Muscle 
A detailed inventory of specimens contained in the Alaska Marine Mammal Tissue Archive is presented in the following pages. The AMMTAP inventory includes the following information: species, meristic information on the animals sampled, sampling location, additional samples taken by other researchers, and the location of these additional samples, as well as other miscellaneous information regarding the individual samples or the animal sampled. Also those specimens for which one subsample has been homogenized and divided into aliquots for analysis are identified. The format used to present this data is explained in Table 4. A list of acronyms used in the inventory is presented in Table 5.

Table 4. AMMTAP Sample Inventory Format

Individual ID No. Sampling Date Geographic Area, Village Latitude/Longitude

$\begin{array}{lllll}\text { Sex Age } & \begin{array}{l}\text { Total Length } \\ \text { in } \mathrm{cm}\end{array} & \begin{array}{l}\text { Fluke Width } \\ \text { in } \mathrm{kg}\end{array} & \begin{array}{l}\text { Axillary Girth } \\ \text { in } \mathrm{cm}\end{array} & \begin{array}{l}\text { Blubber Thickness } \\ \text { in } \mathrm{cm}\end{array}\end{array}$

\begin{tabular}{|c|c|c|c|c|c|}
\hline NBSB Samples & NBSB ID & $\begin{array}{l}\text { Subsample } \\
\text { A weight } \\
\text { in grams }\end{array}$ & $\begin{array}{l}\text { Subsample } \\
\text { B weight } \\
\text { in grams }\end{array}$ & Histology & Sample Homogenization \\
\hline
\end{tabular}

Additional samples collected for other researchers:

Organization and ID number, if appropriate

Present location of samples: list of samples

Table 5. Acronyms used in the AMMTAP sample inventory
ADF \& G - Alaska Department of Fish and Game
NBSB - National Biomonitoring Specimen Bank
NBS DWM - North Slope Borough Department of Wildlife Management
NCRC - National Cancer Research Center
NIST - National Institute of Standards and Technology, Gaithersburg, MD
NMFS - National Marine Fisheries Service, Environmental Conservation Division
NMML - National Marine Mammal Laboratory, NMFS
OAD - Office of Ocean Assessment Division, National Ocean Service
SWR - Southwest Fisheries Science Center, NMFS
$\mathrm{U}$ of $\mathbf{A K}$ - University of Alaska 
RINGED SEAL (Phoca hispida)

\begin{tabular}{|c|c|c|c|c|c|c|}
\hline ANIMAL & & SAMPLING & & & LOCATION & LAT/LONG \\
\hline 692-RGSL-001 & & 11 July 88 & & & $\begin{array}{c}\text { Chukchi Sea, } \\
\text { Barrow }\end{array}$ & $71^{\circ} 19^{\prime} 156^{\circ} 50^{\prime}$ \\
\hline$S E X$ & $\mathrm{AGE}(\mathrm{y})$ & $\begin{array}{l}\text { TOTAT } \\
\text { LENGTH }(\mathrm{cm})\end{array}$ & WEIGHT $(\mathrm{kg})$ & FuKE & $\begin{array}{l}\text { AXIL_ARY } \\
\text { GRTH (cm) }\end{array}$ & $\begin{array}{l}\text { BRLUBBER } \\
\text { MUCKNESS (cm) }\end{array}$ \\
\hline $\mathbf{M}$ & 1 & 99.0 & 19.5 & & 60 & 3.5 \\
\hline $\mathrm{NBSB}$ & \multirow{2}{*}{$\begin{array}{l}\text { NBSB } \\
\text { ID NO. }\end{array}$} & \multicolumn{2}{|c|}{ WEIGHT OF SUBSAMPLES } & & HISTOLOGY & SAMPLE \\
\hline onvingen & & $A(g)$ & $\mathrm{B}(\mathrm{g})$ & & & 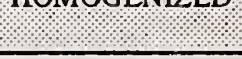 \\
\hline Liver & MM2L021 & 187.5 & 163.5 & & Yes & \\
\hline Kidney & $\mathrm{MM} 2 \mathrm{~K} 022$ & 64.5 & 69.0 & & Yes & \\
\hline Blubber & MM2B023 & 121.0 & 137.0 & & & \\
\hline \multicolumn{7}{|c|}{ ADDITIONAL SAMPLES COLIECTED. } \\
\hline \multicolumn{7}{|c|}{$\begin{array}{l}\text { Bile, blood, liver, muscle to NMFS, Seattle, WA } \\
\text { Stomach content to ADF\&G, NOAA Warehouse, Anchorage, AK } \\
\text { Teeth, histological samples to OAD, Anchorage, AK } \\
\text { Teeth, histological samples transferred from OAD to NIST, NBSB }\end{array}$} \\
\hline
\end{tabular}

\begin{tabular}{|c|c|c|c|c|c|c|}
\hline \multicolumn{7}{|c|}{ RINGED SEAL (Phoca hispida) } \\
\hline $\begin{array}{l}\text { ANIMAL } \\
\text { ID NO. }\end{array}$ & & SAMPLNG & & & LOCATION & LAT / LONG \\
\hline 692-RGSL-002 & & 11 July 88 & & & $\begin{array}{c}\text { Chukchi Sea, } \\
\text { Barrow }\end{array}$ & $71^{\circ} 19^{\prime} 156^{\circ} 50^{\prime}$ \\
\hline SEX & $A G E(y)$ & LENGTH $(\mathrm{cm})$ & WEIGHT $(\mathrm{kg})$ & $\begin{array}{l}\text { FLUKE: } \\
\text { WIDTH } \\
\text { (cm) }\end{array}$ & $\begin{array}{l}\text { AXILLARY } \\
\text { GIRTH (cm) }\end{array}$ & THICKNESS (cm) \\
\hline $\mathbf{F}$ & $\leq 1$ & 75.0 & 12.7 & & 48 & 2.5 \\
\hline WBSB & NBSB & \multicolumn{2}{|c|}{ WEIGHT OF SUBSAMPLES } & & HISTOLOGY & \multirow{2}{*}{ HOMOGENIZED } \\
\hline SAMPLES & $\mathrm{MDO}$ & $\mathrm{A}(\mathrm{g})$ & $\mathrm{B}(\mathrm{g})$ & & & \\
\hline Liver & MM2L024 & 120.5 & 129.5 & & Yes & \\
\hline Kidney & MM2K025 & 45.0 & 42.0 & & Yes & \\
\hline Blubber & MM2B026 & 70.0 & 78.00 & & & \\
\hline \multicolumn{7}{|c|}{ ADDITIONAL SAMPLES COLLECTED. } \\
\hline \multicolumn{7}{|c|}{$\begin{array}{l}\text { Bile, blood, liver, muscle to NMFS, Seattle, WA } \\
\text { Stomach content to ADF\&G, NOAA Warehouse, Anchorage, AK } \\
\text { Histological samples to OAD, Anchorage, AK } \\
\text { Histological samples transferred from OAD to NIST, NBSB }\end{array}$} \\
\hline
\end{tabular}




\begin{tabular}{|c|c|c|c|c|c|c|}
\hline \multicolumn{7}{|c|}{ RINGED SEAL (Phoca hispida) } \\
\hline ANDMAL & & SAMPLNG & & & LOCATION & LAT / LONG \\
\hline 692-RGSL-003 & & 11 July 88 & & & $\begin{array}{c}\text { Chukchi Sea, } \\
\text { Barrow }\end{array}$ & $71^{\circ} 19^{\prime} 156^{\circ} 50^{\prime}$ \\
\hline SEX & AGE $(y)$ & LENGTH (cm) & WEIGHT $(\mathrm{gg})$ & $\begin{array}{l}\text { FLUKE } \\
\text { WIDTH } \\
\text { (cm) }\end{array}$ & $\begin{array}{l}\text { AXILLARY } \\
\text { GIRTH (cm) }\end{array}$ & THICKNESS (cm) \\
\hline $\mathbf{M}$ & 2 & 118.0 & 35.4 & & 70 & 2.5 \\
\hline \multirow{2}{*}{ SAMPLES } & \multirow{2}{*}{ ID NO } & \multicolumn{2}{|c|}{ WEIGHT OF SUBSAMPLES } & & \multirow[t]{2}{*}{ HISTOLOGY } & \multirow{2}{*}{ SAMPLE } \\
\hline & & $A(g)$ & $\mathrm{B}(\mathrm{g})$ & & & \\
\hline Liver & MM2L027 & 155.0 & 150.0 & & yes & \\
\hline Kidney & MM2K028 & 110.0 & 110.5 & & yes & \\
\hline Blubber & MM2B029 & 98.0 & 113.0 & & & \\
\hline \multicolumn{7}{|c|}{ ADDITIONAL SAMPLES COLLECTED. } \\
\hline \multicolumn{7}{|c|}{$\begin{array}{l}\text { Bile, liver, muscle to NMFS, Seattle, WA } \\
\text { Stomach content to ADF\&G, NOAA Warehouse, Anchorage, AK } \\
\text { Teeth, histological samples to OAD, Anchorage, AK } \\
\text { Teeth, histological samples transferred from OAD to NIST, NBSB }\end{array}$} \\
\hline
\end{tabular}

\begin{tabular}{|c|c|c|c|c|c|c|}
\hline \multicolumn{7}{|c|}{ RINGED SEAL (Phoca hispida) } \\
\hline $\begin{array}{l}\text { ANMMAI } \\
\text { ID No. }\end{array}$ & & $\begin{array}{l}\text { SAMPLING } \\
\text { DATE }\end{array}$ & & & LOCATION & LAT / LONG \\
\hline 692-RGSL-004 & & 11 July 88 & & & $\begin{array}{c}\text { Chukchi Sea, } \\
\text { Barrow }\end{array}$ & $71^{\circ} 19^{\prime} 156^{\circ} 50^{\prime}$ \\
\hline SEX & $\mathrm{AGE}(\mathrm{y})$ & LENGTH $(\mathrm{cm})$ & WEIGHT $(\mathrm{kg})$ & $\begin{array}{l}\text { FLUKE } \\
\text { WIDTH } \\
\text { (cm) }\end{array}$ & $\begin{array}{l}\mathrm{AXILLARY} \\
\mathrm{GLRTH}(\mathrm{cm})\end{array}$ & $\begin{array}{l}\text { BLUBBER } \\
\text { THICKNESS (cm) }\end{array}$ \\
\hline $\mathbf{M}$ & 2 & 108.0 & 32.2 & & 84 & 2.0 \\
\hline NBSB & NBSB & \multicolumn{2}{|c|}{ WEIGHT OF SUBSAMPLES } & & \multirow[t]{2}{*}{ HISTOLOGY } & \multirow{2}{*}{$\begin{array}{l}\text { SAMPLE } \\
\text { HOMOGENIZED }\end{array}$} \\
\hline SAMPLES & $10 \mathrm{NO}$ & $A(g)$ & B (g) & & & \\
\hline Liver & MM2L030 & 154.0 & 187.0 & & yes & 27 Feb 89 \\
\hline Kidney & MM2K031 & 106.0 & 113.0 & & yes & $6 \operatorname{Mar} 89$ \\
\hline Blubber & $\mathrm{MM} 2 \mathrm{~B} 032$ & 98.3 & 98.9 & & & 13 Mar 89 \\
\hline \multicolumn{7}{|c|}{ ADDITIONAL SAMPLES COLLECTED. } \\
\hline \multicolumn{7}{|c|}{$\begin{array}{l}\text { Bile, liver, muscle to NMFS, Seattle, WA } \\
\text { Stomach content to ADF\&G, NOAA Warehouse, Anchorage, AK } \\
\text { Teeth, histological samples to OAD, Anchorage, AK } \\
\text { Teeth, histological samples transferred from OAD to NIST, NBSB }\end{array}$} \\
\hline
\end{tabular}


RINGED SEAL (Phoca hispida)

\begin{tabular}{|c|c|c|c|c|c|c|}
\hline ANMMAL & & SAMPLING & & & LOCATION & LAT / LONG \\
\hline 692-RGSL-005 & & 11 July 88 & & & $\begin{array}{c}\text { Chukchi Sea, } \\
\text { Barrow }\end{array}$ & $71^{\circ} 19^{\prime} 156^{\circ} 50^{\prime}$ \\
\hline$S E X$ & $A G E(y)$ & LENGTH $(\mathrm{cm})$ & WEIGHT $(\mathrm{kg})$ & $\begin{array}{l}\text { FLUKE } \\
\mathrm{WTHT} \\
\mathrm{cm}\end{array}$ & $\begin{array}{l}\text { AXILL ARY } \\
\text { GIRTH }(\mathrm{cm})\end{array}$ & RHCLUBBER \\
\hline F & 1 & 84.5 & 15.4 & & 63 & 2.7 \\
\hline \multirow{2}{*}{$\begin{array}{l}\text { NBSB } \\
\text { SAMPLES }\end{array}$} & \multirow{2}{*}{$\begin{array}{l}\mathrm{NBSB} \\
\mathrm{IDNO}\end{array}$} & \multicolumn{2}{|c|}{ WEIGHT OF SUBSAMPLES } & & HISTOLOGY & \multirow{2}{*}{ HOMOGENIZED } \\
\hline & & $A(g)$ & $\mathrm{B}(\mathrm{g})$ & & & \\
\hline Liver & MM2L033 & 127.0 & 130.6 & & yes & \\
\hline Kidney & MM2K034 & 48.0 & 49.0 & & yes & \\
\hline Blubber & MM2B035 & 141.4 & 150.2 & & & \\
\hline \multicolumn{7}{|c|}{ ADDHIONAY SAMPLES COLHECTED. } \\
\hline \multicolumn{7}{|c|}{$\begin{array}{l}\text { Bile, liver, muscle to NMFS, Seattle, WA } \\
\text { Stomach content to ADF\&G, NOAA Warehouse, Anchorage, AK } \\
\text { Teeth, histological samples to OAD, Anchorage, AK } \\
\text { Teeth, histological samples transferred from OAD to NIST, NBSB }\end{array}$} \\
\hline
\end{tabular}

\begin{tabular}{|c|c|c|c|c|c|c|}
\hline \multicolumn{7}{|c|}{ RINGED SEAL (Phoca hispida) } \\
\hline : $\mathrm{DN} \mathrm{No}$ & & SAMPLING & & & LOCATION & LAT / LONG \\
\hline 692-RGSL-006 & & 12 July 88 & & & $\begin{array}{l}\text { Chukchi Sea, } \\
\text { Barrow }\end{array}$ & $71^{\circ} 19^{\prime} 156^{\circ} 50^{\prime}$ \\
\hline SEX & AGE $(\theta)$ & 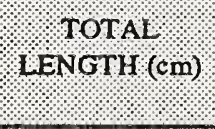 & WEIGHT ( $\mathrm{rg}$ ) & $\begin{array}{l}\text { FLUKE } \\
\text { WIDTH } \\
(\mathrm{cm})\end{array}$ & $\begin{array}{l}\text { AXILLAR Y } \\
\text { GIRTH }(\mathrm{cm})\end{array}$ & THICKNESS (com) \\
\hline $\mathbf{F}$ & $\leq 1$ & 88.0 & 15.4 & & 58 & 2.8 \\
\hline V VBSB & $\mathrm{NBSB}$ & \multicolumn{2}{|c|}{ WEIGHT OF SUBSAMPLES } & & HISTOLOGY & , SAMPLE \\
\hline SAMPEES & & $\mathrm{A}(\mathrm{g})$ & B (g) & & & \\
\hline Liver & MM2L036 & 129.3 & 128.8 & & yes & \\
\hline Kidney & $\mathrm{MM} 2 \mathrm{~K} 037$ & 54.5 & 58.0 & & yes & \\
\hline Blubber & $\mathrm{MM} 2 \mathrm{~B} 038$ & 102.6 & 118.6 & & & \\
\hline \multicolumn{7}{|c|}{ ADDITIONAL SAMTPLES COLLECTED. } \\
\hline \multicolumn{7}{|c|}{$\begin{array}{l}\text { Bile, blood, liver, muscle to NMFS, Seattle, WA } \\
\text { Histological samples to OAD, Anchorage, AK } \\
\text { Histological samples transferred from OAD to NIST, NBSB }\end{array}$} \\
\hline
\end{tabular}


RINGED SEAL (Phoca hispida)

\begin{tabular}{|c|c|c|c|c|c|c|}
\hline ANDMAL & & SAMPLING & & & LOCATION & LAT / LONG \\
\hline 692-RGSL-007 & & 12 July 88 & & & $\begin{array}{c}\text { Chukchi Sea, } \\
\text { Barrow }\end{array}$ & $71^{\circ} 23^{\prime} 156^{\circ} 32^{\prime}$ \\
\hline SEX & $\mathrm{AGE}(y)$ & LENGTH (cm) & WEIGHT $(\mathrm{kg})$ & $\begin{array}{l}\text { FLUKE } \\
\text { WIDTH } \\
\text { (cm) }\end{array}$ & $\begin{array}{l}\text { AXILLARY } \\
\text { GRTH (cm) }\end{array}$ & $\begin{array}{l}\text { BLUBBER } \\
\text { THICKNESS }(\mathrm{cm})\end{array}$ \\
\hline $\mathbf{M}$ & 2 & 94.0 & 23.1 & & 75 & 2.9 \\
\hline \multirow{2}{*}{ SAMPBES } & \multirow{2}{*}{$\mathrm{NBSB}$} & \multicolumn{2}{|c|}{ WEIGHT OF SUBSAMPLES } & & \multirow[t]{2}{*}{ HISTOLOGY } & \multirow{2}{*}{ SAMOGENIZED } \\
\hline & & $A(g)$ & $\mathrm{B}(\mathrm{g})$ & & & \\
\hline Liver & MM2L039 & 165.6 & 162.5 & & yes & \\
\hline Kidney & MM2K040 & 86.6 & 84.8 & & yes & \\
\hline Blubber & MM2B041 & 138.5 & 133.6 & & & \\
\hline \multicolumn{7}{|c|}{ ADDITIONAL SAMPLES COLLECTED. } \\
\hline \multicolumn{7}{|c|}{$\begin{array}{l}\text { Bile, blood, liver, muscle to NMFS, Seattle, WA } \\
\text { Teeth, histological samples to OAD, Anchorage, AK } \\
\text { Teeth, histological samples transferred from OAD to NIST, NBSB }\end{array}$} \\
\hline
\end{tabular}

\begin{tabular}{|c|c|c|c|c|c|c|}
\hline \multicolumn{7}{|c|}{ RINGED SEAL (Phoca hispida) } \\
\hline $\mathrm{ANDMAL}$ & & SAMPLING & & & LOCATION & LAT / LONG \\
\hline 692-RGSL-008 & & 13 July 88 & & & $\begin{array}{c}\text { Chukchi Sea, } \\
\text { Barrow }\end{array}$ & $71^{\circ} 23^{\prime} 156^{\circ} 32^{\prime}$ \\
\hline SEX & $A G E(Y)$ & LENOTH $(\mathrm{cm})$ & WEIGHT $(\mathrm{kg})$ & $\begin{array}{l}\text { FLUKE } \\
\text { WIDTH } \\
\text { (cm) }\end{array}$ & $\begin{array}{l}\text { AXILLARY } \\
\text { GIRTH (cm) }\end{array}$ & $\begin{array}{l}\text { BLUBBER } \\
\text { THICKNESS }(\mathrm{cm})\end{array}$ \\
\hline $\mathbf{M}$ & 2 & 104.0 & 23.1 & & 63.5 & 2.8 \\
\hline WBSB & NBSB & \multicolumn{2}{|c|}{ WEIGHT OF SUBSAMPLES } & & \multirow[t]{2}{*}{ HISTOLOGY } & \multirow{2}{*}{$\begin{array}{l}\text { SAMPLE } \\
\text { HOMOGENIZED }\end{array}$} \\
\hline SAMRLES & $4 \mathrm{Nu}$ & $A(g)$ & B (g) & & & \\
\hline Liver & MM2L042 & 154.2 & 168.6 & & yes & 22 Feb 89 \\
\hline Kidney & $\mathrm{MM} 2 \mathrm{~K} 043$ & 89.5 & 95.5 & & yes & 28 Feb 89 \\
\hline Blubber & MM2B044 & 129.2 & 131.0 & & & $7 \mathrm{Mar} 89$ \\
\hline \multicolumn{7}{|c|}{ ADDITIONAL SAMPLES COLLECTED. } \\
\hline \multicolumn{7}{|c|}{$\begin{array}{l}\text { Bile, blood, liver, muscle to NMFS, Seattle, WA } \\
\text { Stomach content to ADF\&G, NOAA Warehouse, Anchorage, AK } \\
\text { Teeth, histological samples to OAD, Anchorage, AK } \\
\text { Teeth, histological transferred from OAD to NIST, NBSB }\end{array}$} \\
\hline
\end{tabular}




\begin{tabular}{|c|c|c|c|c|c|c|}
\hline \multicolumn{7}{|c|}{ RINGED SEAL (Phoca hispida) } \\
\hline $\begin{array}{l}\text { ANnMAL } \\
\text { IDNO}\end{array}$ & & SAMPLDNG & & & LOCATION & LAT / LONG \\
\hline 692-RGSL-009 & & 14 July 88 & & & $\begin{array}{c}\text { Chukchi Sea, } \\
\text { Barrow }\end{array}$ & $71^{\circ} 22^{\prime} 156^{\circ} 37^{\prime}$ \\
\hline SEX & AGE $(y)$ & IENGTH (cm) & WEIGHT $(\mathrm{kg})$ & $\begin{array}{l}\text { FLUKE } \\
\text { WIDTH } \\
\text { (cm) }\end{array}$ & $\begin{array}{l}\text { AXIL ARY } \\
\text { GIRTH }(\mathrm{cm})\end{array}$ & THICKNESS (cr) \\
\hline & $\leq 1$ & 75.0 & 15.0 & & 63 & 2.4 \\
\hline \multirow{2}{*}{ SAMBB } & \multirow{2}{*}{$\begin{array}{l}\mathrm{NBSB} \\
\mathrm{IDNO}\end{array}$} & \multicolumn{2}{|c|}{ WEIGHT OF SUBSAMPLES } & & \multirow[t]{2}{*}{ HISTOLOGY } & \multirow{2}{*}{$\begin{array}{l}\text { SAMPLE } \\
\text { HOMOGENIZED }\end{array}$} \\
\hline & & A (s) & $\mathrm{B}(\mathrm{g})$ & & & \\
\hline Liver & MM2L045 & 128.1 & 130.8 & & yes & 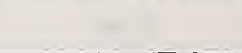 \\
\hline Kidney & MM2K04: & 46.6 & 47.6 & & yes & \\
\hline Blubber & MM2B047 & 86.9 & 94.1 & & & \\
\hline \multicolumn{7}{|c|}{ ADDITIONAL SAMPLES COLLECTED. } \\
\hline \multicolumn{7}{|c|}{$\begin{array}{l}\text { Bile, blood, liver, muscle to NMFS, Seattle, WA } \\
\text { Stomach content to ADF\&G, NOAA Warehouse, Anchorage, AK } \\
\text { Teeth, histological samples to OAD, Anchorage, AK } \\
\text { Histological samples moved from OAD to NIST, NBSB }\end{array}$} \\
\hline
\end{tabular}

\begin{tabular}{|c|c|c|c|c|c|c|}
\hline \multicolumn{7}{|c|}{ RINGED SEAL (Phoca hispida) } \\
\hline ANDMAL & & SAMPLING & & & LOCATION & LAT/LONG \\
\hline 692-RGSL-010 & & 14 July 88 & & & $\begin{array}{c}\text { Chukchi Sea, } \\
\text { Barrow }\end{array}$ & $71^{\circ} 22^{\prime} 156^{\circ} 37^{\prime}$ \\
\hline$S E X$ & AGE $(y)$ & WENGTH $(\mathrm{cm})$ & WEIGHT $(\mathrm{kg})$ & $\begin{array}{l}\text { FLUKE } \\
\text { WIDTH } \\
\text { (cin) }\end{array}$ & GXILARY & THICKNESS (cm) \\
\hline $\mathbf{F}$ & 2 & 98.0 & 25.4 & & 83.5 & 3.0 \\
\hline NABSB & NBSB & \multicolumn{2}{|c|}{ WEIGHT OF SUBSAMPLES } & & HISTOLOGY & \multirow{2}{*}{ HOMOGENIZED } \\
\hline SAMPLES & $10 N O$ & $A(g)$ & $\mathrm{B}(\mathrm{g})$ & & & \\
\hline Liver & MM2L048 & 168.5 & 146.7 & & yes & \\
\hline Kidney & MM2K049 & 78.4 & 76.4 & & yes & \\
\hline Blubber & $\mathrm{MM} 2 \mathrm{~B} 050$ & 146.7 & 161.0 & & & \\
\hline \multicolumn{7}{|c|}{ ADDITIONAL SAMPLES COLLECTED } \\
\hline \multicolumn{7}{|c|}{$\begin{array}{l}\text { Bile, blood, liver to NMFS, Seattle, WA } \\
\text { Teeth, histological samples to OAD, Anchorage, AK } \\
\text { Teeth, histological samples transferred from OAD to NIST, NBSB }\end{array}$} \\
\hline
\end{tabular}


RINGED SEAL (Phoca hispida)

\begin{tabular}{|c|c|c|c|c|c|c|}
\hline ANOMAl & & $\begin{array}{l}\text { SAMPLING } \\
\text { DATE }\end{array}$ & & & LOCATION & LAT / LONG \\
\hline 692-RGSL-011 & & 26 May 89 & & & $\begin{array}{l}\text { Bering Sea, Norton } \\
\text { Sound, Nome }\end{array}$ & $64^{\circ} 19^{\prime} 165^{\circ} 15^{\prime}$ \\
\hline $\mathrm{SEX}$ & $\mathrm{AGE}(\mathrm{)}$ & $\begin{array}{l}\text { LENGTH }(\mathrm{cm}) \\
\text { LOT }\end{array}$ & WEIGHT (kg) & $\begin{array}{l}\text { FLUKE } \\
\text { WIDTH } \\
\text { (cti) }\end{array}$ & $\begin{array}{l}\text { AXILLARY } \\
\text { GIRTH }(\mathrm{cm})\end{array}$ & THICKNESS (cm) \\
\hline $\mathbf{M}$ & 1 & 119.5 & 33.6 & & 82.5 & 4.0 \\
\hline \multirow{2}{*}{ SAMPLES } & \multirow{2}{*}{ NBSB } & \multicolumn{2}{|c|}{ WEIGHT OF SUBSAMPLES } & & \multirow[t]{2}{*}{ HISTOLOGY } & \multirow{2}{*}{ WOMOGENIZED } \\
\hline & & $A(g)$ & $\mathrm{B}(\mathrm{g})$ & & & \\
\hline Liver & MM3L054 & 142.2 & 147.0 & & yes & 10 May 90 \\
\hline Kidney & MM3K055 & 115.1 & 104.5 & & & \\
\hline Blubber & MM3B056 & 128.0 & 145.0 & & & 16 May 90 \\
\hline \multicolumn{7}{|c|}{ ADDITIONALSAMPLES COLLECTED. } \\
\hline \multicolumn{7}{|c|}{$\begin{array}{l}\text { Blood to NMFS, Seattle, WA } \\
\text { Claws, lower jaw, stomach content to ADF\&G, Nome, AK } \\
\text { Histological samples to OAD, Anchorage, Ak } \\
\text { Histological sample (liver only) transferred from OAD to NIST, NBSB }\end{array}$} \\
\hline
\end{tabular}

\begin{tabular}{|c|c|c|c|c|c|c|}
\hline \multicolumn{7}{|c|}{ RINGED SEAL (Phoca hispida) } \\
\hline ANnMAI & & SAMPLING & & & LOCATION & LAT / LONG \\
\hline 692-RGSL-012 & & 26 May 89 & & & $\begin{array}{l}\text { Bering Sea, Norton } \\
\text { Sound, Nome }\end{array}$ & $64^{\circ} 19^{\prime} 165^{\circ} 00^{\prime}$ \\
\hline $\mathrm{SEX}$ & AGE $(y)$ & LENGTH & WOIGHT $(\mathrm{kg})$ & FLUKE & AXIL LARY & THLUCKNBESS $(\mathrm{cm})$ \\
\hline $\mathbf{F}$ & 2 & 124.5 & 36.3 & & 100.0 & 3.5 \\
\hline NBSB & NBSB & \multicolumn{2}{|c|}{ WEIGHT OF SUBSAMPLES } & & \multirow[t]{2}{*}{ HISTOLOGY } & \multirow{2}{*}{$\begin{array}{l}\text { SAMPLE } \\
\text { HOMOGENIZED }\end{array}$} \\
\hline SAMPLES & DDNO. & $A(g)$ & $B(g)$ & & & \\
\hline Liver & MM3L054 & 160.0 & 148.0 & & yes & $21 \mathrm{Jan} 93$ \\
\hline Kidney & MM3K058 & 104.8 & 104.4 & & & \\
\hline Blubber & MM3B059 & 147.0 & 148.0 & & & \\
\hline \multicolumn{7}{|c|}{ ADDRIONAL SAMPLES COLLECTED. } \\
\hline \multicolumn{7}{|c|}{$\begin{array}{l}\text { Bile, blood to NMFS, Seattle, WA } \\
\text { Claws, lower jaw, reproductive organs, stomach contents to ADF\&G, Nome, AK } \\
\text { Histological sample to OAD, Anchorage, AK } \\
\text { Histological sample transferred from OAD to NIST, NBSB }\end{array}$} \\
\hline
\end{tabular}




\begin{tabular}{|c|c|c|c|c|c|c|}
\hline \multicolumn{7}{|c|}{ RINGED SEAL (Phoca hispida) } \\
\hline ANDMAL & & $\begin{array}{l}\text { SAMPLNNG } \\
\text { DATE }\end{array}$ & & & LOCATION & LAT / LONG \\
\hline 692-RGSL-013 & & 31 May 89 & & & $\begin{array}{l}\text { Bering Sea, Norton } \\
\text { Sound, Nome }\end{array}$ & $64^{\circ} 18^{\prime} 165^{\circ} 00^{\prime}$ \\
\hline $\mathrm{SEX}$ & AGE $(y)$ & LENGTH $(\mathrm{cm})$ & WEIGHT $(\mathrm{Kg})$ & $\begin{array}{l}\text { FLUKE } \\
\text { WIDTH } \\
(\mathrm{cm})\end{array}$ & $\begin{array}{l}A X U L_{A R Y} \\
\text { GIRTH (cm) }\end{array}$ & IHICKNESS (cm) \\
\hline $\mathbf{M}$ & 1 & 103.5 & 31.75 & & 79 & 4.5 \\
\hline NBSB & NBSB & \multicolumn{2}{|c|}{ WEIGHT OF SUBSAMPLES } & & HISTOLOGY & sAMPLE \\
\hline SAMPLES & ID NO. & $\mathrm{A}(\mathrm{g})$ & $\mathrm{B}(\mathrm{g})$ & & & HOMOGENIZED \\
\hline Liver & MM3L060 & 95.2 & 110.2 & & yes & 11 May 90 \\
\hline Kidney & MM3K061 & 93.2 & 101.3 & & yes & \\
\hline Blubber & MM3B062 & 149.2 & 167.2 & & & 17 May 90 \\
\hline \multicolumn{7}{|c|}{ ADDIIIONAI SAMPLES COLLECTED. } \\
\hline \multicolumn{7}{|c|}{$\begin{array}{l}\text { Bile, blood, blubber to NMFS, Seattle, WA } \\
\text { Stomach contents, jaws, front flipper to ADF\&G, Nome, AK } \\
\text { Histological samples to OAD, Anchorage, AK } \\
\text { Histological samples transferred from OAD to NIST, NBSB }\end{array}$} \\
\hline
\end{tabular}

\begin{tabular}{|c|c|c|c|c|c|c|}
\hline \multicolumn{7}{|c|}{ RINGED SEAL (Phoca hispida) } \\
\hline $\begin{array}{l}\text { ANMMAT } \\
\text { ID NO }\end{array}$ & & SAMPLING & & & LOCATION & LAT/LONG \\
\hline 692-RGSL-014 & & 31 May 89 & & & $\begin{array}{l}\text { Bering Sea, Norton } \\
\text { Sound, Nome }\end{array}$ & $64^{\circ} 19^{\prime} 164^{\circ} 44^{\prime}$ \\
\hline $\mathrm{SEX}$ & AGE $(8)$ & LENGTH $(\mathrm{cm})$ & WEIGHT $(\mathrm{Tg})$ & FLUKE & $\begin{array}{l}\mathrm{AXIL} / \mathrm{AR} Y \\
\mathrm{GRTH}(\mathrm{cm})\end{array}$ & BHICKNABSS $(\mathrm{cm})$ \\
\hline $\mathbf{M}$ & 1 & 101.0 & 29.5 & & 74 & 3.0 \\
\hline NBSB & NBSB & \multicolumn{2}{|c|}{ WEIGHT OF SUBSAMPLES } & & HISTOLOGY & \multirow{2}{*}{ HOMOGENIZED } \\
\hline SAMPLES & $\mathrm{IDNO}$ & $\mathrm{A}(\mathrm{g})$ & B (g) & & & \\
\hline Liver & MM3L063 & 50.9 & 50.6 & & yes & $9 \operatorname{Dec} 92$ \\
\hline Kidney & MM3K064 & 67.5 & 62.6 & & yes & \\
\hline Blubber & MM3B065 & $10 \AA .0$ & 123.8 & & & \\
\hline \multicolumn{7}{|c|}{ ADDITIONAL SAMPLES COLLECTED. } \\
\hline \multicolumn{7}{|c|}{$\begin{array}{l}\text { Blood, blubber to NMFS, Seattle, WA } \\
\text { Stomach contents, jaws, front flipper to ADF\&G, Nome, AK } \\
\text { Histological samples to OAD, Anchorage, AK } \\
\text { Histological samples transferred from OAD to NIST, NBSB }\end{array}$} \\
\hline
\end{tabular}


RINGED SEAL (Phoca hispida)

\begin{tabular}{|c|c|c|c|c|c|c|}
\hline ANMAN & & SAMPLING & & & LOCATION & LAT / LONG \\
\hline 692-RGSL-015 & 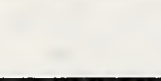 & 31 May 89 & & & $\begin{array}{l}\text { Bering Sea, Norton } \\
\text { Sound, Nome }\end{array}$ & $64^{\circ} 18^{\prime} 165^{\circ} 00^{\prime}$ \\
\hline SEX & AGE $(Y)$ & LENGTH $(\mathrm{cm})$ & WEIGHT $(\mathrm{kg})$ & $\begin{array}{l}\text { FLUKE } \\
\text { WIDTH } \\
(\mathrm{cm})\end{array}$ & $\begin{array}{l}\text { AXIL LARY } \\
\text { GIRTH (cm) }\end{array}$ & $\begin{array}{l}\text { BLUBBER } \\
\text { THICKNESS (cm) }\end{array}$ \\
\hline $\mathbf{F}$ & $\leq 1$ & 83.2 & 20.0 & & 66 & 3.25 \\
\hline \multirow{2}{*}{ NAMSB } & \multirow{2}{*}{$\begin{array}{l}\mathrm{NBSB} \\
\mathrm{IDNO}\end{array}$} & \multicolumn{2}{|c|}{ WEIGHT OF SUBSAMPLES } & & \multirow[t]{2}{*}{ HISTOLOGY } & \multirow{2}{*}{$\begin{array}{l}\text { SAMPLE } \\
\text { HOMOGENIZED }\end{array}$} \\
\hline & & $A(g)$ & $\mathrm{B}(\mathrm{g})$ & & & \\
\hline Liver & MM3L066 & 123.8 & 103.0 & & yes & $17 \operatorname{Dec} 92$ \\
\hline Kidney & MM3K067 & 68.5 & 68.2 & & yes & \\
\hline Blubber & MM3B068 & 110.1 & 103.0 & & & \\
\hline
\end{tabular}

ADDITIONAL SAMPLES COLLECTED:

Blood, bile to NMFS, Seattle, WA

Jaw, front flipper to ADF\&G, Nome, AK

Histological samples to OAD, Anchorage, AK

Histological samples transferred from OAD to NIST, NBSB

\begin{tabular}{|c|c|c|c|c|c|c|}
\hline \multicolumn{7}{|c|}{ RINGED SEAL (Phoca hispida) } \\
\hline $\mathrm{ANDMAL}$ & & $\begin{array}{l}\text { SAMPLANG } \\
\text { DATE }\end{array}$ & & & LOCATION & LAT / LONG \\
\hline 692-RGSL-016 & & 21 May 91 & & & $\begin{array}{l}\text { Bering Sea, Norton } \\
\text { Sound, Nome }\end{array}$ & $64^{\circ} 30^{\prime} 166^{\circ} 10^{\prime}$ \\
\hline SEX & AGE $(y)$ & LENGTH (cm) & WOIGHT $(\mathrm{kg})$ & $\begin{array}{l}\text { FLUKE } \\
\text { WIDTH } \\
\text { (cm) }\end{array}$ & $\begin{array}{l}\text { AXIL ARY } \\
\text { GIRTH }(\mathrm{cm})\end{array}$ & $\begin{array}{l}\text { BLUBBER } \\
\text { THICKNESS (cm) }\end{array}$ \\
\hline $\mathbf{M}$ & $\leq 1$ & 81.5 & 13.6 & & 67 & 4.0 \\
\hline NBSB & NBSB & \multicolumn{2}{|c|}{ WEIGHT OF SUBSAMPLES } & & HISTOLOGY & \multirow{2}{*}{$\begin{array}{l}\text { SAMPLE } \\
\text { HOMOGENIZED }\end{array}$} \\
\hline SANBLE & $201 \times 0$. & $A(g)$ & $\mathrm{B}(\mathrm{g})$ & & & \\
\hline Liver & MM5L155 & 90.4 & 70.6 & & yes & $21 \operatorname{Dec} 92$ \\
\hline Kidney & MM5K156 & 80.5 & 30.3 & & yes & \\
\hline Blubber & MM5B157 & 150.2 & 148.2 & & & \\
\hline \multicolumn{7}{|c|}{ ADDITIONAL SAMPLES COLLECTED: } \\
\hline \multicolumn{7}{|c|}{$\begin{array}{l}\text { Heart, muscle to U of AK, Fairbanks, AK, ID \# AF0751 } \\
\text { Jawbone with teeth. histological samples to OAD, Anchorage, AK } \\
\text { Jawbone with teeth, histological samples transferred from OAD to NIST, NBSB }\end{array}$} \\
\hline
\end{tabular}




\begin{tabular}{|c|c|c|c|c|c|c|}
\hline \multicolumn{7}{|c|}{ RINGED SEAL (Phoca hispida) } \\
\hline ANMMAL & & SAMPL NNG & & & LOCATION & LAT lLONG \\
\hline 692-RGSL-017 & & 21 May 91 & & & $\begin{array}{l}\text { Bering Sea, Norton } \\
\text { Sound, Nome }\end{array}$ & $64^{\circ} 30^{\prime} 166^{\circ} 10^{\prime}$ \\
\hline SEX & $\mathrm{AGE}(\mathrm{Y})$ & LENGTH $(\mathrm{cm})$ & WEIGHT $(\mathrm{kg})$ & $\begin{array}{l}\text { FLUKE } \\
\text { WIDTH }\end{array}$ & $\begin{array}{l}\text { AXILLARY } \\
\text { GRTH }(\mathrm{cm})\end{array}$ & $\begin{array}{l}\text { BLUBBER } \\
\text { THICKNESS (cm) }\end{array}$ \\
\hline $\mathbf{F}$ & Mature & 113 & 40.8 & & 92 & 7.0 \\
\hline NBSB & NBSB & \multicolumn{2}{|c|}{ WEIGHT OF SUBSAMPLES } & & HISTOLOGY & \multirow{2}{*}{ HOMOGENLZED } \\
\hline SAMPLES & DNO. & $A(g)$ & $\mathrm{B}(\mathrm{g})$ & & & \\
\hline Liver & MM5L158 & 176.5 & 175.4 & & yes & $6 \operatorname{Jan} 93$ \\
\hline Kidney & MM5K159 & 97.6 & 93.1 & & yes & \\
\hline Blubber & MM5B159 & 145.8 & 158.5 & & & \\
\hline \multicolumn{7}{|c|}{ ADDITIONAL SAMPLES COLLECTED. } \\
\hline \multicolumn{7}{|c|}{$\begin{array}{l}\text { Heart, muscle, liver to } U \text { of AK, Fairbanks, AK, ID \# AF0752 } \\
\text { Jawbone with teeth, histological samples to OAD, Anchorage, AK } \\
\text { Jawbone with teeth, histological samples transferred from OAD to NIST, NBSB } \\
\text { Extra jar of liver to NIST, NBSB }\end{array}$} \\
\hline
\end{tabular}

\begin{tabular}{|c|c|c|c|c|c|c|}
\hline \multicolumn{7}{|c|}{ RINGED SEAL (Phoca hispida) } \\
\hline ANMAL & & $\begin{array}{l}\text { SAMPLING } \\
\text { DATE }\end{array}$ & & & LOCATION & LAT I LONG \\
\hline 692-RGSL-018 & & 22 May 91 & & & $\begin{array}{l}\text { Bering Sea, Norton } \\
\text { Sound, Nome }\end{array}$ & $64^{\circ} 09^{\prime} 165^{\circ} 26^{\prime}$ \\
\hline $\mathrm{SEX}$ & AGE $(y)$ & WENGTH $(\mathrm{cm})$ & WEIGHT $(\mathrm{kg})$ & FUUK & GIRTH $(\mathrm{cm})$ & THICKNESS (cm) \\
\hline $\mathbf{F}$ & 1 & 87 & 21.8 & & 75 & 2.0 \\
\hline NASB & NBSB & \multicolumn{2}{|c|}{ WEIGHT OF SUBSAMPLES } & & HISTOLOGY & \multirow{2}{*}{ HOMOGENIZED } \\
\hline SAMPLES & $10 \mathrm{NO}$ & $A(g)$ & $\mathrm{B}(\mathrm{g})$ & & & \\
\hline Liver & MM5L161 & 162.8 & 173.0 & & yes & 7 Jan 93 \\
\hline Kidney & MM5K162 & 68.5 & 71.5 & & yes & \\
\hline Blubber & MM5B163 & 151.2 & 148.1 & & & \\
\hline \multicolumn{7}{|c|}{ ADDIIONAL SAMPLES COLLECTED } \\
\hline \multicolumn{7}{|c|}{$\begin{array}{l}\text { Muscle, liver to U of AK, Fairbanks, AK, ID \# AF0753 } \\
\text { Jawbone with teeth, histological samples to OAD, Anchorage, AK } \\
\text { Jawbone with teeth, histological samples transferred from OAD to NIST, NBSB }\end{array}$} \\
\hline
\end{tabular}


RINGED SEAL (Phoca hispida)

\begin{tabular}{|c|c|c|c|c|c|c|}
\hline ANM No. & & SAMPLING & & & LOCATION & LAT / LONG \\
\hline 692-RGSL-019 & 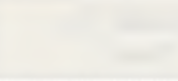 & 9 July 91 & & & $\begin{array}{c}\text { Chukchi Sea, } \\
\text { Barrow }\end{array}$ & $71^{\circ} 18.7^{\prime} 156^{\circ} 53.2^{\prime}$ \\
\hline SEX & AGE $(y)$ & LENGTH $(\mathrm{cm})$ & WEIGHT $(\mathrm{kg})$ & $\begin{array}{l}\text { FLURE } \\
\text { WIDTH } \\
(\mathrm{cm})\end{array}$ & $\begin{array}{l}\text { AXILLARY } \\
\text { GRTH (cm) }\end{array}$ & $\begin{array}{l}\text { BLUBBER } \\
\text { THUCKNESS }(\mathrm{cm})\end{array}$ \\
\hline $\mathbf{M}$ & $\leq 1$ & 77 & 13.6 & & 64 & 2.5 \\
\hline \multirow{2}{*}{ SAMPLES } & \multirow{2}{*}{$\begin{array}{l}\mathrm{NBSB} \\
\mathrm{ID} \mathrm{NO}\end{array}$} & \multicolumn{2}{|c|}{ WEIGHT OF SUBSAMPLES } & & \multirow[t]{2}{*}{ HISTOLOGY } & \multirow{2}{*}{ HOMOGENIZED } \\
\hline & & $A(g)$ & $\mathrm{B}(\mathrm{g})$ & & & \\
\hline Liver & MM5L167 & 127.2 & 123.7 & & yes & \\
\hline Kidney & MM5K168 & 47.9 & 46.3 & & yes & \\
\hline Blubber & MM5B169 & 127.9 & 134.2 & & & \\
\hline \multicolumn{7}{|c|}{ ADDITIONAL SAMPLES COLIECTED. } \\
\hline \multicolumn{7}{|c|}{$\begin{array}{l}\text { Muscle, liver to U of AK, Fairbanks, AK, ID \# AF0757 } \\
\text { Jawbone, histological samples to OAD, Anchorage, AK } \\
\text { Jawbone, histological samples transferred from OAD to NIST, NBSB }\end{array}$} \\
\hline
\end{tabular}

\begin{tabular}{|c|c|c|c|c|c|c|}
\hline \multicolumn{7}{|c|}{ RINGED SEAL (Phoca hispida) } \\
\hline $\begin{array}{l}\text { ANMAI } \\
\text { ID NO. }\end{array}$ & & SAMPLING & & & LOCATION & LAT / LONG \\
\hline 692-RGSL-020 & & 9 July 91 & & & $\begin{array}{c}\text { Chukchi Sea, } \\
\text { Barrow }\end{array}$ & $71^{\circ} 18.7^{\prime} 156^{\circ} 53.2^{\prime}$ \\
\hline SEX & $A G E(y)$ & LENGTH (cm) & $\begin{array}{l}\text { TOTAL } \\
\text { WEIGHT }(\mathrm{kg})\end{array}$ & $\begin{array}{l}\text { FLUKE } \\
\text { WIDTH } \\
\text { (cm) }\end{array}$ & $\begin{array}{l}\text { AXILLARY } \\
\text { GIRTH (cm) }\end{array}$ & $\begin{array}{l}\text { BLUBBER } \\
\text { THICKNESS }(\mathrm{cm})\end{array}$ \\
\hline $\mathbf{M}$ & $11-12$ & 119.4 & 41.7 & & 92 & 2.5 \\
\hline \multirow{2}{*}{ SAMPLES } & \multirow{2}{*}{$\begin{array}{l}\mathrm{NBSB} \\
\mathrm{WNO}\end{array}$} & \multicolumn{2}{|c|}{ WEIGHT OF SUBSAMPLES } & & \multirow[t]{2}{*}{ HISTOLOGY } & \multirow{2}{*}{$\begin{array}{l}\text { SAMPLE } \\
\text { HOMOGENIZED }\end{array}$} \\
\hline & & $A(g)$ & B (g) & & & \\
\hline Liver & MM5L170 & 158.7 & 173.7 & & yes & \\
\hline Kidney & MM5K171 & 123.2 & 123.2 & & yes & \\
\hline Blubber & MM5B172 & 163.5 & 158.9 & & & \\
\hline \multicolumn{7}{|c|}{ ADDITIONAL SAMPLES COLLECTED } \\
\hline \multicolumn{7}{|c|}{$\begin{array}{l}\text { Muscle, liver to U of AK, Fairbanks, AK, ID \# AF0758 } \\
\text { Blood serum to ADF\&G, Fairbanks, AK } \\
\text { Jawbone, histological samples to OAD, Anchorage, AK } \\
\text { Jawbone, histological samples transferred from OAD to N }\end{array}$} \\
\hline
\end{tabular}


RINGED SEAL (Phoca hispida)

\begin{tabular}{|c|c|c|c|c|c|c|}
\hline ANIMAL & & SAMPLNNG & & & LOCATION & $\mathrm{LAT} / \mathrm{LONG}$ \\
\hline 692-RGSL-021 & & 10 July 91 & & & $\begin{array}{c}\text { Chukchi Sea, } \\
\text { Barrow }\end{array}$ & $71^{\circ} 18.7^{\prime} 156^{\circ} 53.2^{\prime}$ \\
\hline SEX & $\mathrm{AGEU}$ & IENGTH $(\mathrm{cm})$ & WEIGHT $(\mathrm{kg})$ & $\begin{array}{l}\mathrm{FL} U \mathrm{UK} \\
\mathrm{WIDTH} \\
(\mathrm{cm})\end{array}$ & $\begin{array}{l}\text { AXIL LARY } \\
\text { GIRTH }(\mathrm{cm})\end{array}$ & THICKNESS (cm) \\
\hline $\mathbf{M}$ & $8-9$ & 108 & 36.3 & & 85.5 & 2.0 \\
\hline NBSB & \multirow{2}{*}{ NBSB } & \multicolumn{2}{|c|}{ WEIGHT OF SUBS AMPLES } & & HISTOLOOY & SAMPLE \\
\hline SAMPEES & & $\mathrm{A}(\mathrm{g})$ & $\mathrm{B}(\mathrm{g})$ & & & HUMUGENLED \\
\hline Liver & MM5L173 & 173.0 & 177.3 & & yes & \\
\hline Kidney & MM5K174 & 132.0 & 129.3 & & yes & \\
\hline Blubber & MM5B175 & 94.4 & 22.2 & & & \\
\hline & & & & & & \\
\hline
\end{tabular}

ADDITIONAL SAMPLES COLLECTED:

Muscle, liver to $U$ of AK, Fairbanks, AK, D \# AF0760

Jawbone, histological samples to $\mathrm{OAD}$, Anchorage, $\mathrm{AK}$

Jawbone, histological samples transferred from OAD to NIST, NBSB

\begin{tabular}{|c|c|c|c|c|c|c|}
\hline \multicolumn{7}{|c|}{ RINGED SEAL (Phoca hispida) } \\
\hline ANDMAI & & SAMPLING & & & LOCATION & LAT / LONG \\
\hline 692-RGSL-022 & & 10 July 91 & & & $\begin{array}{c}\text { Chukchi Sea, } \\
\text { Barrow }\end{array}$ & $71^{\circ} 18.7^{\prime} 156^{\circ} 53.2^{\prime}$ \\
\hline SEX & $(A G E(y)$ & l. TONGTH (cm) & $\begin{array}{l}\text { TOTAL } \\
\text { WEIGHT ( kg) }\end{array}$ & $\begin{array}{l}\text { FLUKE } \\
\text { WIDTH } \\
(\mathrm{cm})\end{array}$ & $\begin{array}{l}\text { AXILLARY } \\
\text { GIRTH (cm) }\end{array}$ & $\begin{array}{l}\text { BLUBBER } \\
\text { THICKNESS (cor) }\end{array}$ \\
\hline $\mathbf{M}$ & 5 & 97.8 & 22.2 & & 70 & 2.0 \\
\hline \multirow{2}{*}{ SAMPLES } & \multirow{2}{*}{ NBSB No. } & \multicolumn{2}{|c|}{ WEIGHT OF SUBSSAMPLES . } & & \multirow[t]{2}{*}{ HISTOLOGY } & \multirow{2}{*}{$\begin{array}{l}\text { SAMPLE } \\
\text { HOMOGENIZED }\end{array}$} \\
\hline & & $A(g)$ & $B(g)$ & & & \\
\hline Liver & MM5L176 & 137.2 & 157.3 & & yes & \\
\hline Kidney & MM5K177 & 94.4 & 56.6 & & yes & \\
\hline Blubber & MM5B178 & 119.5 & 121.8 & & & \\
\hline \multicolumn{7}{|c|}{ ADDITIONAL SAMPLES COLLECTED. } \\
\hline \multicolumn{7}{|c|}{$\begin{array}{l}\text { Muscle, liver to U of AK, Fairbanks, AK, ID \# AF0761 } \\
\text { Jawbone, histological samples to OAD, Anchorage, AK } \\
\text { Jawbone, histological samples transferred from OAD to NIST, NBSB }\end{array}$} \\
\hline
\end{tabular}




\begin{tabular}{|c|c|c|c|c|c|c|}
\hline \multicolumn{7}{|c|}{ RINGED SEAL (Phoca hispida) } \\
\hline $\begin{array}{l}\text { ANMMAL } \\
\text { ID NO. }\end{array}$ & & SAMPLING & & & LOCATION & LAT / LONG \\
\hline 692-RGSL-023 & & 10 July 91 & & & $\begin{array}{l}\text { Chukchi Sea, } \\
\text { Barrow }\end{array}$ & $71^{\circ} 18.7^{\prime} 156^{\circ} 53.2^{\prime}$ \\
\hline SEX & AGE $(y)$ & LENGTH $(\mathrm{cm})$ & $\begin{array}{l}\text { TOTAL } \\
\text { WEIGHT }(\mathrm{kg})\end{array}$ & $\begin{array}{l}\text { FLUKE } \\
\text { WTDTH } \\
(\mathrm{cm})\end{array}$ & $\begin{array}{l}\text { AXILLARY } \\
\text { GIRTH (cm) }\end{array}$ & $\begin{array}{c}\text { BLUBBER } \\
\text { THICKNESS }(\mathrm{cm})\end{array}$ \\
\hline $\mathbf{F}$ & $5-6$ & 103.9 & 29.0 & & 76.5 & 2.3 \\
\hline NBSB & NBSB & \multicolumn{2}{|c|}{ WEIGHT OF SUBSAMPLES } & & HISTOLOGY & \multirow{2}{*}{$\begin{array}{l}\text { SAMPLE } \\
\text { HOMOGENIZED }\end{array}$} \\
\hline SAMPLES & ID No. & $A(g)$ & $\mathrm{B}(\mathrm{g})$ & & & \\
\hline Liver & MM5L179 & 189.1 & 169.5 & & yes & \\
\hline Kidney & MM5K180 & 97.6 & 29.0 & & yes & \\
\hline Blubber & MM5B181 & 149.3 & 112.5 & & & \\
\hline \multicolumn{7}{|c|}{ ADDITIONAL SAMPLES COLLECTED } \\
\hline \multicolumn{7}{|c|}{$\begin{array}{l}\text { Muscle, liver to } \mathrm{U} \text { of } \mathrm{AK} \text {, Fairbanks, } \mathrm{AK}, \mathrm{ID} \# \mathrm{AF} 0762 \\
\text { Jawbone, histological samples to OAD, Anchorage, AK } \\
\text { Jawbone, histological samples transferred from OAD to NIST, NBSB }\end{array}$} \\
\hline
\end{tabular}

\begin{tabular}{|c|c|c|c|c|c|c|}
\hline \multicolumn{7}{|c|}{ RINGED SEAL (Phoca hispida) } \\
\hline ANDMAL & & $\begin{array}{l}\text { SAMPLING } \\
\text { DATE }\end{array}$ & & & LOCATION & LAT / LONG \\
\hline 692-RGSL-024 & & 11 July 91 & & & $\begin{array}{c}\text { Chukchi Sea, } \\
\text { Barrow }\end{array}$ & $71^{\circ} 20.1^{\prime} 156^{\circ} 41.7^{\prime}$ \\
\hline SEX & AGE $(Y)$ & LENGTH $(\mathrm{cm})$ & WEIGHT (kg) & $\begin{array}{l}\text { FLUKE } \\
\text { WIDTH } \\
\text { (cm) }\end{array}$ & AXIL LARY & $\begin{array}{l}\text { BLUBBER } \\
\text { THICKNESS }(\mathrm{cm})\end{array}$ \\
\hline $\mathbf{M}$ & 6 & 118 & 38.1 & & 87 & 2.7 \\
\hline NBSB & NBSB & \multicolumn{2}{|c|}{ WEIGHT OF SUBSAMPLES } & & \multirow[t]{2}{*}{ HISTOLOGY } & \multirow{2}{*}{$\begin{array}{l}\text { SAMPLE } \\
\text { HOMOGENIZED }\end{array}$} \\
\hline SAMPLES & $10 \mathrm{NO}$ & $A(g)$ & $\mathrm{B}(\mathrm{g})$ & & & \\
\hline Liver & MM5L182 & 170.7 & 176.0 & & yes & \\
\hline Kidney & MM5K183 & 137.1 & 130.1 & & yes & \\
\hline Blubber & MM5B184 & 149.1 & 145.3 & & & \\
\hline \multicolumn{7}{|c|}{ ADDITIONAL SAMPLES COLLECTED: } \\
\hline \multicolumn{7}{|c|}{$\begin{array}{l}\text { Muscle, liver to U of AK, Fairbanks, AK, ID \# AF0763 } \\
\text { Blood serum to ADF\&G, Fairbanks, AK } \\
\text { Jawbone, histological samples to OAD, Anchorage, AK } \\
\text { Jawbone, histological samples transferred from OAD to N }\end{array}$} \\
\hline
\end{tabular}




\begin{tabular}{|c|c|c|c|c|c|c|}
\hline \multicolumn{7}{|c|}{ RINGED SEAL (Phoca hispida) } \\
\hline $\begin{array}{l}\text { ANMMAL } \\
\text { ID NO. }\end{array}$ & & $\begin{array}{l}\text { SAMPLNNG } \\
\text { DATE }\end{array}$ & & & LOCATION & LAT / LONG \\
\hline 692-RGSL-025 & & 11 July 91 & & & $\begin{array}{c}\text { Chukchi Sea, } \\
\text { Barrow }\end{array}$ & $71^{\circ} 20.1^{\prime} 156^{\circ} 41.7^{\prime}$ \\
\hline SEX & $\mathrm{AGE}(\mathrm{Y})$ & lENGTH (cm) & WEIGHT $(\mathrm{kg})$ & $\begin{array}{l}\mathrm{FLUKE} \\
\mathrm{WIDTH} \\
\mathrm{cm}\end{array}$ & $\begin{array}{l}\text { AXUL AR } \\
\text { GRTH (cm) }\end{array}$ & THCKNUESS (cm) \\
\hline $\mathbf{F}$ & $1-2$ & 104 & 27.2 & & 77.5 & 2.4 \\
\hline NBSB & NBSB & \multicolumn{2}{|c|}{ WEIGHT OF SUBSAMPIES } & & HISTOLOGY & SAMPLE \\
\hline SAMPLES: & ID NO. & $A(g)$ & $\mathrm{B}(\mathrm{g})$ & & & HOMOGENIZED \\
\hline Liver & MM5L185 & 160.7 & 167.3 & & yes & \\
\hline Kidney & MM5K186 & 67.0 & 75.3 & & yes & \\
\hline Blubber & MM5B187 & 160.5 & 152.8 & & & \\
\hline \multicolumn{7}{|c|}{ ADDITIONAI SAMPLES COLLECTED. } \\
\hline \multicolumn{7}{|c|}{$\begin{array}{l}\text { Muscle, liver to U of AK, Fairbanks, AK, ID \# AF0764 } \\
\text { Blood serum to ADF\&G, Fairbanks, AK } \\
\text { Jawbone, histological samples to OAD, Anchorage, AK } \\
\text { Jawbone, histological samples transferred from OAD to NIST, NBSB }\end{array}$} \\
\hline
\end{tabular}

\begin{tabular}{|c|c|c|c|c|c|c|}
\hline \multicolumn{7}{|c|}{ RINGED SEAL (Phoca hispida) } \\
\hline ANMMAI & & SAMPLING & & & LOCATION & HAT/LONG \\
\hline 692-RGSL-026 & & 12 July 91 & & & $\begin{array}{c}\text { Chukchi Sea, } \\
\text { Barrow }\end{array}$ & $71^{\circ} 24.1^{\prime} 156^{\circ} 31.5^{\prime}$ \\
\hline SEX & $\mathrm{AGE}(\mathrm{O})$ & LENGTH $(\mathrm{cm})$ & WEIGHT $(\mathrm{kg})$ & $\begin{array}{l}\text { FLUKE } \\
\text { WIDTH } \\
\text { (coin) }\end{array}$ & $\begin{array}{l}\mathrm{AXH} \mathrm{ARY} \\
\mathrm{GLTH}(\mathrm{cm})\end{array}$ & $\begin{array}{l}\text { BLUUBBER } \\
\text { THICKNESS (cm) }\end{array}$ \\
\hline $\mathbf{M}$ & $5-6$ & 109 & 36.3 & & 72 & 2.0 \\
\hline NBSB & NBSB & \multicolumn{2}{|c|}{ WEIGHT OF SUBSAMPLES } & & HISTOLOGY & \multirow{2}{*}{ 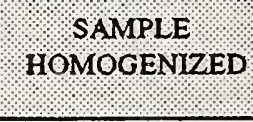 } \\
\hline SAMPLES & $\mathrm{IDNO}$ & $A(g)$ & B (g) & & & \\
\hline Liver & MM5L188 & 139.1 & 123.4 & & yes & \\
\hline Kidney & MM5K189 & 87.5 & 36.3 & & yes & \\
\hline Blubber & MM5B190 & 154.9 & 154.2 & & & \\
\hline \multicolumn{7}{|c|}{ ADDITIONAI SAMPLES COLLECTED. } \\
\hline \multicolumn{7}{|c|}{$\begin{array}{l}\text { Muscle, liver to U of AK, Fairbanks, AK, UD \# AF0765 } \\
\text { Jawbone, histological samples to OAD, Anchorage, AK } \\
\text { Jawbone, histological samples transferred from OAD to NIST, NBSB }\end{array}$} \\
\hline
\end{tabular}




\begin{tabular}{|c|c|c|c|c|c|c|}
\hline \multicolumn{7}{|c|}{ RINGED SEAL (Phoca hispida) } \\
\hline ANnMAL & & sAMPLNNG & & & LOCATION & LAT / LONG \\
\hline 692-RGSL-027 & & 15 July 91 & & & $\begin{array}{c}\text { Chukchi Sea, } \\
\text { Barrow }\end{array}$ & $71^{\circ} 18.5^{\prime} 156^{\circ} 53.2^{\prime}$ \\
\hline SEX & AGE $(y)$ & LENGTH $(\mathrm{cm})$ & $\begin{array}{l}\text { TOTAL } \\
\text { WEIGHT }(\mathrm{kg})\end{array}$ & $\begin{array}{l}\text { FLUKE } \\
\text { WIDTH } \\
(\mathrm{cm})\end{array}$ & $\begin{array}{l}\text { AXILLARY } \\
\text { GIRTH }(\mathrm{cm})\end{array}$ & $\begin{array}{l}\text { BLUBBER } \\
\text { THICKNESS (cm) }\end{array}$ \\
\hline $\mathbf{M}$ & $2-3$ & 101.6 & 23.6 & & 74 & 2.0 \\
\hline NBSB & NBSB & \multicolumn{2}{|c|}{ WEIGHT OF SUBSAMPLES } & & HISTOLOGY & \multirow{2}{*}{$\begin{array}{l}\text { SAMPLE } \\
\text { HOMOGENIZED }\end{array}$} \\
\hline SAMPLES & ID NO. & $A(g)$ & $\mathrm{B}(\mathrm{g})$ & & & \\
\hline Liver & MM5L191 & $15: .4$ & 162.6 & & yes & \\
\hline Kidney & MM5L192 & 87.4 & 83.5 & & yes & \\
\hline Blubber & MM5B193 & 151.4 & 152.8 & & & \\
\hline \multicolumn{7}{|c|}{ ADDITIONAL SAMPLES COLLECTED. } \\
\hline \multicolumn{7}{|c|}{$\begin{array}{l}\text { Muscle, liver to U of AK, Fairbanks, AK, ID \# AF0759 } \\
\text { Blood serum to ADF\&G, Fairbanks, AK } \\
\text { Jawbone, histological samples to OAD, Anchorage, AK } \\
\text { Jawbone, histological samples transferred from OAD to NIST, NBSB }\end{array}$} \\
\hline
\end{tabular}

\begin{tabular}{|c|c|c|c|c|c|c|}
\hline \multicolumn{7}{|c|}{ RINGED SEAL (Phoca hispida) } \\
\hline ANMAL & & SAMPLING & & & LOCATION & LAT / LONG \\
\hline 692-RGSL-028 & & 15 July 91 & & & $\begin{array}{c}\text { Chukchi Sea, } \\
\text { Barrow }\end{array}$ & $71^{\circ} 18.5^{\prime} 156^{\circ} 53.2^{\prime}$ \\
\hline SEX & AGE $(\mathrm{y})$ & LENGTH (cm) & WEIGHT $(\mathrm{kg})$ & $\begin{array}{l}\text { FLUKE } \\
\text { WIDTH } \\
(\mathrm{cm})\end{array}$ & $\begin{array}{l}\text { AXILLARY } \\
\text { GIRTH }(\mathrm{cm})\end{array}$ & $\begin{array}{l}\text { BLUBBER } \\
\text { THICKNESS (cm) }\end{array}$ \\
\hline $\mathbf{F}$ & 9 & 110 & 21.8 & & 90.7 & 2.5 \\
\hline NBSB & NBSB & \multicolumn{2}{|c|}{ WEIGHT OF SUBSAMPLES } & & \multirow[t]{2}{*}{ HISTOLOGY } & \multirow{2}{*}{ HOMOGENIZED } \\
\hline SAMPLES & $\mathrm{WNO}$. & $A(g)$ & $\mathrm{B}(\mathrm{g})$ & & & \\
\hline Liver & MM5L191 & 170.7 & 152.8 & & yes & \\
\hline Kidney & MM5K195 & 92.0 & 21.8 & & yes & \\
\hline Blubber & MM5B196 & 140.7 & 153.5 & & & \\
\hline \multicolumn{7}{|c|}{ ADDITIONAL SAMPLES COLLECTED } \\
\hline \multicolumn{7}{|c|}{$\begin{array}{l}\text { Muscle, liver to U of AK, Fairbanks, AK, ID \# AF0766 } \\
\text { Blood serum to ADF\&G, Fairbanks, AK } \\
\text { Jawbone, histological samples to OAD, Anchorage, AK } \\
\text { Jawbone, histological samples transferred from OAD to N }\end{array}$} \\
\hline
\end{tabular}




\begin{tabular}{|c|c|c|c|c|c|c|}
\hline \multicolumn{7}{|c|}{ RINGED SEAL (Phoca hispida) } \\
\hline ANDMAL & & SAMPLING & & & LOCATION & LAT / LONG \\
\hline 692-RGSL-029 & & 11 May 93 & & & $\begin{array}{l}\text { Bering Sea, Norton } \\
\text { Sound, Nome }\end{array}$ & $64^{\circ} 30^{\prime} 166^{\circ} 10^{\prime}$ \\
\hline SEX & $A G E(y)$ & LENGTH $(\mathrm{cm})$ & WEIOHT $(\mathrm{kg})$ & $\begin{array}{l}\mathrm{FLUKE} \\
\mathrm{wiDTH} \\
(\mathrm{cm})\end{array}$ & $\begin{array}{l}\mathrm{AXILLARY} \\
\mathrm{GIRTH}(\mathrm{cm})\end{array}$ & THICKNESS (cm) \\
\hline $\mathbf{M}$ & $1-2$ & 106.7 & & & 86 & 3.4 \\
\hline NBSB & NBSB & \multicolumn{2}{|c|}{ WEIGHT OF SUBSAMPLES } & & HISTOLOGY & SAMPLE \\
\hline SAMPLES & $10 \mathrm{NO}$ & $A(g)$ & $\mathrm{B}(\mathrm{g})$ & & & HOMOGENIZED \\
\hline Liver & MM5L226 & 92.5 & 86.7 & & yes & $13 \operatorname{Dec} 93$ \\
\hline Kidney & MM5K227 & 81.0 & 58.9 & & yes & \\
\hline Blubber & MM5E226 & 152.7 & 154.5 & & & \\
\hline \multicolumn{7}{|c|}{ ADDITIONAI SAMPLES COLLECTED. } \\
\hline \multicolumn{7}{|c|}{$\begin{array}{l}\text { Blubber, liver, kidney to NMFS, Seattle, WA } \\
\text { Front claws, jawbones, histological samples to NIST, NBSB }\end{array}$} \\
\hline
\end{tabular}

\begin{tabular}{|c|c|c|c|c|c|c|}
\hline \multicolumn{7}{|c|}{ RINGED SEAL (Phoca hispida) } \\
\hline ANMMAI & & SAMPLING & & & LOCATION & LAT $/$ LONG \\
\hline 692-RGSL-030 & & 12 May 93 & & & $\begin{array}{l}\text { Bering Sea, Norton } \\
\text { Sound, Nome }\end{array}$ & $64^{\circ} 30^{\prime} 166^{\circ} 10^{\prime}$ \\
\hline $\mathrm{SEX}$ & AGE $(y)$ & WENGTH $(\mathrm{cm})$ & WEIGHT $(\mathrm{Kg})$ & $\begin{array}{l}\text { FLUKE } \\
\text { WIDTH } \\
\text { (cm) }\end{array}$ & GIRTH $(\mathrm{cm})$ & THICKNESS $(\mathrm{cm})$ \\
\hline $\mathbf{M}$ & & 165 & & & 109 & 6 \\
\hline / NBSB & NBSB & \multicolumn{2}{|c|}{ WEIGHT OF SUBSAMPLES } & & \multirow[t]{2}{*}{ HISTOLOGY } & \multirow{2}{*}{ HOMOGENIZED } \\
\hline SAMPLES & $10 \mathrm{NO}$ & $\mathrm{A}(\mathrm{g})$ & $\mathrm{B}(\mathrm{g})$ & & & \\
\hline Liver & MM7L229 & 129.1 & 99.3 & & yes & $14 \operatorname{Dec} 93$ \\
\hline Kidney & MM7K230 & 162.1 & 146.0 & & yes & \\
\hline Blubber & MM7B231 & 154.3 & 153.1 & & & \\
\hline \multicolumn{7}{|c|}{ ADDITIONAL SAMPLES COLHECTED } \\
\hline \multicolumn{7}{|c|}{$\begin{array}{l}\text { Blubber, liver, kidney to NMFS, Seattle, WA } \\
\text { Front claws, jawbones, histological samples to NIST, NBSB }\end{array}$} \\
\hline
\end{tabular}




\begin{tabular}{|c|c|c|c|c|c|c|}
\hline \multicolumn{7}{|c|}{ RINGED SEAL (Phoca hispida) } \\
\hline ANIMALI & & SAMPLNG & & & LOCATION & LAT / LONG \\
\hline 692-RGSL-031 & & 12 May 93 & & & $\begin{array}{l}\text { Bering Sea, Norton } \\
\text { Sound, Nome }\end{array}$ & $64^{\circ} 23166^{\circ} 25^{\prime}$ \\
\hline SEX & $\mathrm{AGE}(Y)$ & LENGTH $(\mathrm{cm})$ & WEIGHT $(\mathbf{k g})$ & $\begin{array}{l}\text { FLUKE } \\
\text { wIDTH } \\
(\mathrm{cm})\end{array}$ & $\begin{array}{l}\text { AXILLARY } \\
\text { GIRTH }(\mathrm{cm})\end{array}$ & THICKNESS $(\mathrm{cm})$ \\
\hline $\mathbf{M}$ & $1-2$ & 89 & & & 71 & 4.0 \\
\hline NBSB & NBSB & \multicolumn{2}{|c|}{ WEIGHT OF SUBSAMPLES } & & \multirow[t]{2}{*}{ HISTOLOGY } & \multirow{2}{*}{ SAMPLE } \\
\hline SAMPLES & II NOF & $A(g)$ & $\mathrm{B}(\mathrm{g})$ & & & \\
\hline Liver & MM7L232 & 59.0 & 77.8 & & yes & $15 \operatorname{Dec} 93$ \\
\hline Kidney & MM7K233 & 72.2 & 79.3 & & yes & \\
\hline Blubber & MM5B234 & 142.0 & 139.5 & & & 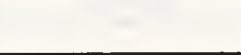 \\
\hline \multicolumn{7}{|c|}{ ADDITIONAL SAMTLLES COLLECTED. } \\
\hline \multicolumn{7}{|c|}{$\begin{array}{l}\text { Blubber, liver, kidney to NMFS, Seattle, WA } \\
\text { Front claws, jawbone, histological samples to NIST, NBSB }\end{array}$} \\
\hline
\end{tabular}

\begin{tabular}{|c|c|c|c|c|c|c|}
\hline \multicolumn{7}{|c|}{ RINGED SEAL (Phoca hispida) } \\
\hline $\begin{array}{l}\text { ANMMAL } \\
\text { ID NO }\end{array}$ & & SAMPLING & & & LOCATION & LAT / LONG \\
\hline 692-RGSL-032 & & 3 June 93 & & & $\begin{array}{l}\text { Bering Sea, Norton } \\
\text { Sound, Nome }\end{array}$ & $64^{\circ} 23^{\prime} 166^{\circ} 25^{\prime}$ \\
\hline SEX & $A G E(Y)$ & LENGTH (cm) & WEIGHT (kg) & $\begin{array}{l}\text { FLUTKE } \\
\text { WIDTH } \\
(\mathrm{cm})\end{array}$ & $\begin{array}{l}\text { AXILLARY } \\
\text { GIRTH }(\mathrm{cm})\end{array}$ & $\begin{array}{l}\text { BLUBBER } \\
\text { THICKNESS }(\mathrm{cm})\end{array}$ \\
\hline $\mathbf{M}$ & $\leq 1$ & 79 & & & 60 & 6.3 \\
\hline NBSB & NBSB & \multicolumn{2}{|c|}{ WEIGHT OF SUBSAMPLES } & & \multirow[t]{2}{*}{ HISTOLOGY } & \multirow{2}{*}{$\begin{array}{l}\text { SAMPLE } \\
\text { HOMOGENIZED }\end{array}$} \\
\hline SAMFLO & $w 1 \times u$ & $A(g)$ & $\mathrm{B}(\mathrm{g})$ & & & \\
\hline Liver & MM7L247 & 65.3 & 55.5 & & yes & $20 \operatorname{Dec} 93$ \\
\hline Kidney & MM7K248 & 34.8 & & & & \\
\hline Blubber & MM7B249 & 75.1 & 85.4 & & & \\
\hline \multicolumn{7}{|c|}{ ADDIIIONAL SAMPLES COLLECTED. } \\
\hline \multicolumn{7}{|c|}{ Jawbone to NIST, NBSB } \\
\hline
\end{tabular}




\begin{tabular}{|c|c|c|c|c|c|c|}
\hline \multicolumn{7}{|c|}{ RINGED SEAL (Phoca hispida) } \\
\hline ANMMAI. & & SAMPLING & & & IOCATION & LAT / LONG \\
\hline 692-RGSL-033 & ( & 19 May 94 & & & Nome, Cape Nome & $63^{\circ} 59^{\prime} 165^{\circ} 39^{\prime}$ \\
\hline sex & AGE ( $)$ & LENGTAL (cm) & WEIGHT ( $\mathrm{Tg}$ ) & $\begin{array}{l}\text { FLUKE } \\
\text { WIDTH } \\
\text { (cm) }\end{array}$ & $\begin{array}{l}\text { AXILLARY } \\
\text { GIRTH (cm) }\end{array}$ & . \\
\hline M & & 111 & 88.6 & & 97.5 & 4.0 \\
\hline \multirow{2}{*}{$\begin{array}{l}\text { NBSB } \\
\text { SAMPLES } \\
.\end{array}$} & \multirow{2}{*}{ iD No } & \multicolumn{2}{|c|}{ WEIGHT OF SUBSAMPLES } & & \multirow[t]{2}{*}{ HISTOLOGY } & \multirow{2}{*}{ HOMOGENIZED } \\
\hline & & $A(g)$ & $\mathrm{B}(\mathrm{g})$ & & & \\
\hline Liver & MM9L276 & 157.0 & 119.0 & & yes & \\
\hline Kidney & MM9K277 & 86.9 & 88.6 & & yes & \\
\hline Blubber & MM9B278 & 142.6 & 119.8 & & & \\
\hline \multicolumn{7}{|c|}{ ADDTIONAL SAMPLES COLLECTED. } \\
\hline \multicolumn{7}{|c|}{$\begin{array}{l}\text { Blubber, liver to NWFS, Seattle, WA } \\
\text { Jawbone with teeth, histological samples to NIST, NBSB }\end{array}$} \\
\hline
\end{tabular}

\begin{tabular}{|c|c|c|c|c|c|c|}
\hline \multicolumn{7}{|c|}{ RINGED SEAL (Phoca hispida) } \\
\hline ID No & & SAMPLING & & & LOCATION & LAT / LONG \\
\hline 692-RGSL-034 & & 21 May 94 & & & Nome, Cape Nome & $63^{\circ} 59^{\prime} 165^{\circ} 39^{\prime}$ \\
\hline SEX & AGE $(y)$ & I. TONATH $(\mathrm{cm})$ & WEIGHT (kg) & $\begin{array}{l}\text { FLURE } \\
\text { WIDTH } \\
\text { (cm) }\end{array}$ & $\begin{array}{l}\text { AXIILARY } \\
\text { GIRTH (cm) }\end{array}$ & THICKNESS $(\mathrm{cm})$ \\
\hline $\mathbf{M}$ & & 92.7 & 20.9 & & 76.2 & 2.5 \\
\hline \multirow{2}{*}{ SAMPLES } & \multirow{2}{*}{ NBSB } & \multicolumn{2}{|c|}{ WEIGHT OF SUBSAMPLES } & & \multirow[t]{2}{*}{ HISTOLOGY } & \multirow{2}{*}{ HOMOGENIZED } \\
\hline & & $A(g)$ & B (g) & & & \\
\hline Liver & MM9L279 & 80.4 & 57.6 & & yes & \\
\hline Kidney & MM9K280 & 57.1 & 54.8 & & yes & \\
\hline Blubber & MM9B281 & 157.0 & 143.5 & & & \\
\hline \multicolumn{7}{|c|}{ ADDITIONAL SAMPLES COLLECTED. } \\
\hline \multicolumn{7}{|c|}{$\begin{array}{l}\text { Jawbone to NIST, NBSB } \\
\text { Blubber to NWFS, Seattle, WA }\end{array}$} \\
\hline
\end{tabular}




\begin{tabular}{|c|c|c|c|c|c|c|}
\hline \multicolumn{7}{|c|}{ RINGED SEAL (Phoca hispida) } \\
\hline ANMMAL & & SAMPLNNG & & & LOCATION & LAT / LONG \\
\hline 692-RGSL-035 & & 21 May 94 & & & Nome, Cape Nome & $63^{\circ} 59^{\prime} 165^{\circ} 39^{\prime}$ \\
\hline SEX & AGE $(Y)$ & LENGTH $(\mathrm{cm})$ & WEIGHT $(\mathrm{kg})$ & $\begin{array}{l}\text { FU UKKE } \\
\text { WIDTH } \\
(\mathrm{cm})\end{array}$ & $\begin{array}{l}\text { AXIL LARY } \\
\text { GIRTH }(\mathrm{cm})\end{array}$ & $\begin{array}{l}\text { BLUBBER } \\
\text { THICKNESS }(\mathrm{cm})\end{array}$ \\
\hline $\mathbf{F}$ & & 90.8 & 28.1 & & 81.3 & 3.5 \\
\hline NBSB & NBSB & \multicolumn{2}{|c|}{ WEIGHT OF SUBSAMPLES } & & \multirow[t]{2}{*}{ HISTOLOGY } & \multirow{2}{*}{ HOMOGENIZED } \\
\hline SAMPLES & $\mathrm{IDNO}$ & $\mathrm{A}(\mathrm{g})$ & $B(g)$ & & & \\
\hline Liver & MM9L282 & 67.1 & 94.2 & & yes & \\
\hline Kidney & MM9K283 & 71.8 & 70.5 & & yes & \\
\hline Blubber & MM9B284 & 137.7 & 143.3 & & & \\
\hline \multicolumn{7}{|c|}{ ADDITIONAL SAMPLES COLLECTED. } \\
\hline \multicolumn{7}{|c|}{ Jawbone with teeth, histological samples to NIST, NBSB } \\
\hline
\end{tabular}

\begin{tabular}{|c|c|c|c|c|c|c|}
\hline \multicolumn{7}{|c|}{ RINGED SEAL (Phoca hispida) } \\
\hline ANM NOI & & SAMPLING & & & LOCATION & LAT / LONG \\
\hline 692-RGSL-036 & & 22 May 94 & & & Nome, Cape Nome & $63^{\circ} 59^{\prime} 165^{\circ} 39^{\prime}$ \\
\hline SEX & $A G E(8)$ & LENGTH $(\mathrm{cm})$ & WEIGHT $(\mathrm{kg})$ & $\begin{array}{l}\text { FLUKE } \\
\text { WIDTH } \\
(\mathrm{cm})\end{array}$ & $\begin{array}{l}\text { GXIL ARY } \\
\text { GIRT }(\mathrm{cm})\end{array}$ & THICKNESS (cm) \\
\hline $\mathrm{F}$ & & 110.5 & 94.2 & & 93.3 & 3.75 \\
\hline \multirow{2}{*}{ SAMPLES } & \multirow{2}{*}{$\mathrm{NBSB}$} & \multicolumn{2}{|c|}{ WEIGHT OF SUBSAMPLES } & & \multirow[t]{2}{*}{ HISTOLOGY } & \multirow{2}{*}{ HOMOGENIZED } \\
\hline & & $A(g)$ & B (g) & & & \\
\hline Liver & MM9L285 & 96.3 & 102.1 & & yes & \\
\hline Kidney & MM9K286 & 67.1 & 90.3 & & yes & \\
\hline Blubber & MM9B287 & 110.5 & 163.2 & & & \\
\hline \multicolumn{7}{|c|}{ ADDITIONAL SAMPLES COLLECTED: } \\
\hline \multicolumn{7}{|c|}{$\begin{array}{l}\text { Jawbone and teeth, histologies to NIST, NBSB } \\
\text { Blubber, liver to NWFS, Seattle, WA }\end{array}$} \\
\hline
\end{tabular}




\begin{tabular}{|c|c|c|c|c|c|c|}
\hline \multicolumn{7}{|c|}{ RINGED SEAL (Phoca hispida) } \\
\hline AD NO. & & $\begin{array}{l}\text { SAMPLING } \\
\text { DATE }\end{array}$ & & & LOCATION & LAT / LONG \\
\hline 692-RGSL-037 & & 15 July 94 & & & Barrow & \\
\hline SEX & AGE $(v)$ & $\begin{array}{l}\text { TOTAL } \\
\text { LENGTH }(\mathrm{cm}) \text { ? }\end{array}$ & WEIGHT (kg) & $\begin{array}{l}\text { FLUKE } \\
\text { WIDTH } \\
(\mathrm{cm})\end{array}$ & 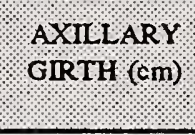 & THICKNESS (cm) \\
\hline $\mathbf{M}$ & $1 \mathrm{yr}$ & 121.0 & 18.1 & & 83.2 & 3.2 \\
\hline NBSB , . & NBSB & \multicolumn{2}{|c|}{ WEIGHT OF SUBSAMPLES } & & \multirow[t]{2}{*}{ HISTOLOGY } & \multirow{2}{*}{ HOMOGENIZED } \\
\hline SAMPLES & IDNO. & $A(g)$ & $\mathrm{B}(\mathrm{g})$ & & & \\
\hline Liver & MM9L307 & 109.0 & 108.8 & & yes & \\
\hline Kidney & MM9K3II & 126.1 & 108.8 & & yes & \\
\hline Blubber & MM9E3I0 & 98.6 & 116.2 & & & \\
\hline \multicolumn{7}{|c|}{ ADDTHONAL SAMPLES COLLECTED. } \\
\hline \multicolumn{7}{|c|}{$\begin{array}{l}\text { Jawbone, liver, histological samples to NIST, NBSB } \\
\text { Liver to Greg O'Corry-Crowe, NMFS, SWR }\end{array}$} \\
\hline
\end{tabular}

\begin{tabular}{|c|c|c|c|c|c|c|}
\hline \multicolumn{7}{|c|}{ RINGED SEAL (Phoca hispida) } \\
\hline ANM Not: & & SAMPLING & & & LOCATION & LAT / LONG \\
\hline 692-RGSL-038 & & 15 July 94 & & & Barrow & \\
\hline SEX & AGE (y) & 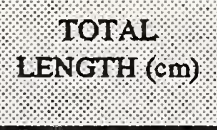 & $\begin{array}{l}\text { TOTAL }, \text { W } \\
\text { WEIGHT (kg) }\end{array}$ & $\begin{array}{l}\text { FLURE } \\
\text { WIDTH } \\
(\mathrm{cm})\end{array}$ & $\begin{array}{l}\text { AXULARY } \\
\text { GIRTH (cm) }\end{array}$ & THICKNIESS (com) \\
\hline $\mathbf{M}$ & 7 claw rings & 185 & 20.4 & & 86.2 & 3.2 \\
\hline , NBSB & NBSB , & \multicolumn{2}{|c|}{ WEIGHT OF SUBSAMPLES } & & \multirow[t]{2}{*}{ HISTOLOGY } & \multirow{2}{*}{ Y SOMOGENIZEO } \\
\hline SAlines & Wot: & A (g) & $\mathrm{B}(\mathrm{g})$ & & & \\
\hline Liver & MM9L310 & 116.7 & 109.5 & & yes & \\
\hline Kidney & MM9K311 & 132.0 & 128.9 & & & \\
\hline Blubber & MM9B312 & 107.6 & 110.1 & & & \\
\hline \multicolumn{7}{|c|}{ ADDTIONAL SAMPLES COLLECTED } \\
\hline \multicolumn{7}{|c|}{$\begin{array}{l}\text { Muscle, histology to NIST, NBSB } \\
\text { Muscle to Greg O'Corry-Crowe, NMFS, SWR } \\
\text { Heart study - isolated coronary arteries to Dr. Robert Elsner, }\end{array}$} \\
\hline
\end{tabular}




\begin{tabular}{|c|c|c|c|c|c|c|}
\hline \multicolumn{7}{|c|}{ RINGED SEAL (Phoca hispida) } \\
\hline ANIMALI & & SAMPLING & & & LOOCATION & LAT / LONG \\
\hline 692-RGSL-039 & & 15 July 94 & & & Barrow & \\
\hline $\mathrm{SEX}$ & $\operatorname{AGE}(y)$ & LENGTH $(\mathrm{cm})$ & WEIGHT $(\mathrm{kg})$ & $\begin{array}{l}\text { FLUKE } \\
\text { WIDTH } \\
\text { (cm) }\end{array}$ & $\begin{array}{l}\text { AXILLARY } \\
\text { GIRTH }(\mathrm{cm})\end{array}$ & $\begin{array}{l}\text { BLUBBER } \\
\text { THICKNESS (cm) }\end{array}$ \\
\hline $\mathbf{F}$ & 8 claw rings & 122.0 & 22.7 & & 95.0 & 4.0 \\
\hline , NBSB & NBSB & \multicolumn{2}{|c|}{ WEIGHT OF SUBSAMPLES } & & HISTOLOGY & SAMPLE \\
\hline SAMPLES & ID NO. & $A(g)$ & $\mathrm{B}(\mathrm{g})$ & & & HOMOGENIZED \\
\hline Liver & MM9L313 & 175.0 & 159.8 & & yes & \\
\hline Kidney & MM9K314 & 110.9 & 95.2 & & yes & \\
\hline Blubber & MM9B315 & 154.8 & 157.6 & & & \\
\hline \multicolumn{7}{|c|}{ ADDIIIONAL SAMPLES COLLECTED, } \\
\hline \multicolumn{7}{|c|}{$\begin{array}{l}\text { Jawbone, histological samples to NIST, NBSB } \\
\text { Blubber to NWFS, Seattle, WA } \\
\text { Heart study - isolated coronary arteries to Dr. Robert Elsner, U of AK, Fairbanks, AK }\end{array}$} \\
\hline
\end{tabular}

\begin{tabular}{|c|c|c|c|c|c|c|}
\hline \multicolumn{7}{|c|}{ RINGED SEAL (Phoca hispida) } \\
\hline ANM NO & & SAMPLING & & & LOCATION & LAT $/$ LONG \\
\hline 692-RGSL-040 & & 18 July 94 & & & Barrow & \\
\hline SEX & AGE $(y)$ & LENGTH $(\mathrm{cm})$ & WEIGHT $(\mathrm{kg})$ & $\begin{array}{l}\text { PUUK } \\
\text { WIDTH } \\
\text { (cm) }\end{array}$ & AXIL ARY & $\begin{array}{l}\text { BLUBBER } \\
\text { THICKNESS (cm) }\end{array}$ \\
\hline $\mathbf{M}$ & 8 claw rings & 94.0 & & & 72.0 & 2.4 \\
\hline WBSB & NBSB & \multicolumn{2}{|c|}{ WEIGHT OF SUBSAMPLES } & & HISTOLOGY & \multirow{2}{*}{$\begin{array}{c}\text { SAMPLE } \\
\text { HOMOGENIZED }\end{array}$} \\
\hline SAMPLES & $10 \mathrm{NO}$ & $A(g)$ & $\mathrm{B}(\mathrm{g})$ & & & \\
\hline Liver & MM9L316 & 106.1 & 101.6 & & yes & \\
\hline Kidney & MM9K317 & 106.6 & 91.09 & & yes & \\
\hline Blubber & MM9B318 & 93.5 & 106.6 & & & \\
\hline \multicolumn{7}{|c|}{ ADDITIONAL SAMPLES COLLECTED. } \\
\hline \multicolumn{7}{|c|}{$\begin{array}{l}\text { Jawbone, bag of unlabeled tissue, histological samples to NIST, NBSB } \\
\text { Blood for Stress Proteins to Dr. Michael Castellini, U of AK, Fairbanks, AK } \\
\text { Blood, heart study - isolated coronary arteries to Dr. Robert Elsner, U of AK, Fairbanks, AK }\end{array}$} \\
\hline
\end{tabular}




\begin{tabular}{|c|c|c|c|c|c|c|}
\hline \multicolumn{7}{|c|}{ RINGED SEAL (Phoca hispida) } \\
\hline ANDLAL & & SAMPLING & & & LOCATION & LAT / LONG \\
\hline 692-RGSL-041 & & 18 July 94 & & & Barrow & \\
\hline sEx. & AGE $(3)$ & $\begin{array}{l}\text { TOTAL } \\
\text { LENGTH (cm) }\end{array}$ & , TOTAHT (kg) & $\begin{array}{l}\text { FLUKE } \\
\text { WIDTH } \\
(\mathrm{cm})\end{array}$ & $\begin{array}{l}\text { AXILLARY } \\
\text { GIRTH (cm) }\end{array}$ & THICKNESS (cm) \\
\hline $\mathbf{M}$ & 7 claw rings & 109.0 & & & 82.3 & 2.7 \\
\hline NBSB & NBSB & \multicolumn{2}{|c|}{ WEIGHT OF SUBSAMPLES } & & Histologr & , \\
\hline SAMPLES & & $A(g)$ & B (g) & & & \\
\hline Liver & MM9L319 & 120.5 & 112.2 & & yes & \\
\hline Kidney & MM9K320 & 77.0 & 80.0 & & yes & \\
\hline Blubber & MM9B321 & 115.7 & 111.1 & & & \\
\hline \multicolumn{7}{|c|}{ ADDIIIONAL SAMPLES COLLECTED. } \\
\hline \multicolumn{7}{|c|}{$\begin{array}{l}\text { Jawbone, histological samples to NIST, NBSB } \\
\text { Blood for Stress Protein to Dr. Michael Castellini, U of AK, Fairbanks, AK } \\
\text { Blood, heart study - isolated coronary arteries to Dr. Robert Elsner, U of AK, Fairt }\end{array}$} \\
\hline
\end{tabular}

\begin{tabular}{|c|c|c|c|c|c|c|}
\hline \multicolumn{7}{|c|}{ RINGED SEAL (Phoca hispida) } \\
\hline ANIMAI & & SAMPLING & & & LOCATION & LAT / LONG \\
\hline 692-RGSL-042 & & 19 July 94 & & & Barrow & \\
\hline SEX & AGE (y) & 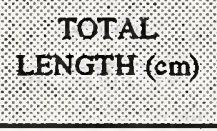 & $\begin{array}{l}\text { TOTAL }, ~ \\
\text { WEIGHT (Ig) }\end{array}$ & $\begin{array}{l}\text { FLURE } \\
\text { WDTH } \\
.(\mathrm{cm})\end{array}$ & GXIRTH (cm) & THICKNESS (cm) \\
\hline $\mathbf{M}$ & 7 claw rings & 101.0 & 31.8 & & 82.3 & 3.0 \\
\hline NBSB : . & $\mathrm{NBSB}$ & \multicolumn{2}{|c|}{ WEIGHT OF SUBSAMPLES } & & HISTOLOG Y & . SAMPLE \\
\hline SAMPLES & WONo. & $A(g)$ & $\mathrm{B}(\mathrm{g})$ & & & HOMOGENIZED \\
\hline Liver & MM9L322 & 128.6 & 105.0 & & yes & \\
\hline Kidney & MM9K323 & 93.7 & 80.8 & & yes & \\
\hline Blubber & MM9B324 & 156.5 & 148.1 & & & \\
\hline \multicolumn{7}{|c|}{ ADDTTIONAL SAMPLES COLLECTED } \\
\hline \multicolumn{7}{|c|}{$\begin{array}{l}\text { Jawbone, histological samples to NIST, NBSB } \\
\text { Blood for Stress Proteins to Dr. Michael Castellini, U of AK, Fairbanks, AK } \\
\text { Blood to Dr. Robert Elsner, U of AK, Fairbanks, AK }\end{array}$} \\
\hline
\end{tabular}


HARBOR SEAL (Phoca vitulina)

\begin{tabular}{|c|c|c|c|c|c|c|}
\hline ANMMAL & & $\begin{array}{l}\text { SAMPLING } \\
\text { DATE }\end{array}$ & & & LOCATION & LAT / LONG \\
\hline 692-HBSL-001 & & 10 March 90 & & & $\begin{array}{l}\text { Prince William } \\
\text { Sound, New Year } \\
\text { Island, Chenega }\end{array}$ & $60^{\circ} 10.9^{\prime} 147^{\circ} 55.3^{\prime}$ \\
\hline $\mathrm{SEX}$ & $A G E(y)$ & LENGTH $(\mathrm{cm})$ & WEIGHT $(\mathrm{kg})$ & $\begin{array}{l}\text { FLUKE } \\
\text { WIDTH } \\
(\mathrm{cm})\end{array}$ & $\begin{array}{l}\text { AXILLARY } \\
\text { GIRTH (cm) }\end{array}$ & $\begin{array}{c}\text { BLUBBER } \\
\text { THICKNESS (cm) }\end{array}$ \\
\hline $\mathbf{M}$ & 1 & 105.4 & 28 & & 74.5 & 4.0 \\
\hline \multirow{2}{*}{ SAMBLES } & \multirow{2}{*}{ NBSB } & \multicolumn{2}{|c|}{ WEIGHT OF SUBSAMPLES } & & \multirow[t]{2}{*}{ HISTOLOGY } & \multirow{2}{*}{$\begin{array}{l}\text { SAMPLE } \\
\text { HOMOGENIZED }\end{array}$} \\
\hline & & $A(g)$ & $\mathrm{B}(\mathrm{g})$ & & & \\
\hline Liver & MM4L083 & 165.3 & 163.1 & & yes & \\
\hline Kidney & MM4K093 & 72.05 & 37.3 & & yes & \\
\hline Blubber & MM4B085 & 136.0 & 153.4 & & & \\
\hline \multicolumn{7}{|c|}{ ADDITIONAL SAMIPLES COLLECTED. } \\
\hline $\begin{array}{l}\text { Bile, liver, blubbe } \\
\text { Jawbone, histologi }\end{array}$ & $\begin{array}{l}\text { le, lung, rigl } \\
\text { ples to NIST }\end{array}$ & rum, cerebellum & FS, Seattle, WA & & & \\
\hline
\end{tabular}

\begin{tabular}{|c|c|c|c|c|c|c|}
\hline \multicolumn{7}{|c|}{ HARBOR SEAL (Phoca vitulina) } \\
\hline $\begin{array}{l}\text { ANMMAI } \\
\text { ID NO. }\end{array}$ & & $\begin{array}{l}\text { SAMPLING } \\
\text { DATE }\end{array}$ & & & LOCATION & LAT / LONG \\
\hline 692-HBSL-002 & & 22 April 90 & & & $\begin{array}{l}\text { Prince William } \\
\text { Sound, Galena } \\
\text { Bay, Tatilek }\end{array}$ & $60^{\circ} 55.45^{\prime} 146^{\circ} 36.2$ \\
\hline SEX & AGE $(y)$ & LENGTH (cm) & WEIGHT $(\mathrm{kg})$ & $\begin{array}{l}\text { FLUKE } \\
\text { WDTH } \\
\text { (cm) }\end{array}$ & $\begin{array}{l}\text { AXILLARY } \\
\text { GIRTH }(\mathrm{cm})\end{array}$ & $\begin{array}{l}\text { BLUBBER } \\
\text { THICKNESS (cm) }\end{array}$ \\
\hline $\mathbf{F}$ & 2 & 125.1 & 47.6 & & 94.5 & 3.8 \\
\hline \multirow{2}{*}{ SAMBSB } & \multirow{2}{*}{$\mathrm{NBSB}$} & \multicolumn{2}{|c|}{ WEIGHT OF SUBSAMPLES } & & \multirow[t]{2}{*}{ HISTOLOGY } & \multirow{2}{*}{$\begin{array}{l}\text { SAMPLE } \\
\text { HOMOGENIZED }\end{array}$} \\
\hline & & $A(g)$ & B (g) & & & \\
\hline Liver & $\mathrm{MM} 4 \mathrm{LO} 92$ & 138.1 & 139.05 & & yes & \\
\hline Kidney & MM4K093 & 120.3 & 94.3 & & yes & \\
\hline Blubber & MM4B094 & 149.4 & 151.85 & & & \\
\hline \multicolumn{7}{|c|}{ ADDITIONAL SAMPLES COLLECTED } \\
\hline \multicolumn{7}{|c|}{$\begin{array}{l}\text { Bile, liver, blubber, kidney, muscle, lung, ovaries to NMFS, Seattle, WA } \\
\text { Jawbone, histological samples to OAD, Anchorage, AK } \\
\text { Jawbone, histological samples transferred from OAD to NIST, NBSB }\end{array}$} \\
\hline
\end{tabular}




\begin{tabular}{|c|c|c|c|c|c|c|}
\hline \multicolumn{7}{|c|}{ HARBOR SEAL (Phoca vitulina) } \\
\hline ANMMAL & & SAMPLING & & & LOCATION & LAT / LONG \\
\hline 692-HBSL-003 & & 22 April 90 & & & Prince William & $60^{\circ} 55.45^{\prime} 146^{\circ} 36.2^{\prime}$ \\
\hline SEX & $\mathrm{AGE}(\mathrm{Y})$ & LENGTAL & WEIGHT $(\mathrm{kg})$ & $\begin{array}{l}\text { FUTKE } \\
\text { WIDTH } \\
(\mathrm{cm})\end{array}$ & $\begin{array}{l}\text { AXILLARY } \\
\text { GIRTH (cm) }\end{array}$ & THICKNESS $(\mathrm{cm})$ \\
\hline $\mathbf{M}$ & 2 & 125.1 & 39.9 & & 85.0 & 3.0 \\
\hline NBSB & NBSB & \multicolumn{2}{|c|}{ WEIGHT OF SUBSAMPLES } & & HISTOLOGY & \multirow{2}{*}{ HOMOGENIZED } \\
\hline SAMPLES & $10 \mathrm{NO}$ & $4(\mathrm{~g})$ & $\mathrm{B}(\mathrm{g})$ & & & \\
\hline Liver & MM4L089 & 160.35 & 164.4 & & yes & \\
\hline Kidney & MM4K090 & 80.2 & 112.9 & & yes & \\
\hline Blubber & MM4B091. & 125.4 & 105.3 & & & \\
\hline \multicolumn{7}{|c|}{ ADDITIONAL SAMPLES COLLECTED. } \\
\hline \multicolumn{7}{|c|}{$\begin{array}{l}\text { Bile, liver, blubber, kidney, muscle, lung to NMFS, Seattle, WA } \\
\text { Jawbone, histological samples to OAD, Anchorage, AK } \\
\text { Jawbone, histological samples transferred from OAD to NIST, NBSB }\end{array}$} \\
\hline
\end{tabular}

\begin{tabular}{|c|c|c|c|c|c|c|}
\hline \multicolumn{7}{|c|}{ HARBOR SEAL (Phoca vitulina) } \\
\hline ANMMAI & & SAMPLING & & & LOCATION & LAT L LONG \\
\hline 692-HBSL-004 & & 15 June 94 & & & $\begin{array}{c}\text { Anchorage, AK } \\
\text { Sound, Galena } \\
\text { Bay, Tatilek }\end{array}$ & \\
\hline $\operatorname{SEX}$ & AGE $(6)$ & LENGTH $(\mathrm{cm})$ & WETGHT $(\mathrm{kg})$ & $\begin{array}{l}\text { FLUKE } \\
\text { WIDTH } \\
(\mathrm{cm})\end{array}$ & $\begin{array}{l}\mathrm{AXILARY} \\
\mathrm{GIRTH}(\mathrm{cm})\end{array}$ & THICKNESS (cm) \\
\hline$F$ & & 109.0 & 18.1 & & 94.5 & 3.5 \\
\hline \multirow{2}{*}{ NAMPB } & \multirow{2}{*}{$\begin{array}{l}\mathrm{NBSB} \\
\mathrm{IDNO}\end{array}$} & \multicolumn{2}{|c|}{ WEIGHT OF SUBSAMPLES } & & \multirow[t]{2}{*}{ HISTOLOGY } & \multirow{2}{*}{$\begin{array}{l}\text { SAMPLE } \\
\text { HOMOGENIZED }\end{array}$} \\
\hline & & A (g) & $\mathrm{B}(\mathrm{g})$ & & & \\
\hline Liver & MM9L325 & 164.0 & 163.2 & & yes & \\
\hline Kidney & MM9K326 & 59.7 & 55.0 & & yes & \\
\hline Blubber & MM9B327 & 156.4 & 151.2 & & & \\
\hline \multicolumn{7}{|c|}{ ADDITIONAL SAMPLES COLLECTED. } \\
\hline \multicolumn{7}{|c|}{ Histological samples to NIST, NBSB } \\
\hline
\end{tabular}




\begin{tabular}{|c|c|c|c|c|c|c|}
\hline \multicolumn{7}{|c|}{ SPOTTED SEAL (Phoca largha) } \\
\hline ANINAL & & SAMPLANG & & & LOCATION & LAT / LONG \\
\hline 692-SPSL-001 & & 22 May 91 & & & $\begin{array}{l}\text { Bering Sea, Norton } \\
\text { Sound, Nome }\end{array}$ & $64^{\circ} 09^{\prime} 165^{\circ} 26^{\prime}$ \\
\hline$S E X$ & $A G E(y)$ & LENGTH (cm) & WEIGHT $(\mathrm{kg})$ & $\begin{array}{l}\text { FLUKE } \\
\text { WIDTH } \\
\text { (cm) }\end{array}$ & $\begin{array}{l}\text { AXIILARY } \\
\text { GIRTH }(\mathrm{cm})\end{array}$ & THICKNESS (cm) \\
\hline $\mathbf{F}$ & Mature & 157.5 & & & 98.4 & 8.5 \\
\hline NBSB & NBSB & \multicolumn{2}{|c|}{ WEIGHT OF SUBSAMPLES } & & \multirow[t]{2}{*}{ HISTOLOGY } & \multirow{2}{*}{ SAMPLE } \\
\hline SAMPLES & $\mathrm{ID} \mathrm{NO}$ & $A(g)$ & $\mathrm{B}(\mathrm{g})$ & & & \\
\hline Liver & MM5L164 & 143.5 & 145.2 & & yes & 9 Jan 93 \\
\hline Kidney & MM5K165 & 161.8 & 161.7 & & yes & \\
\hline Blubber & MM5B166 & 153.7 & 159.3 & & & \\
\hline \multicolumn{7}{|c|}{ ADDITIONAL SAMPLES COLLECTED. } \\
\hline \multicolumn{7}{|c|}{$\begin{array}{l}\text { Liver, muscle to } \mathrm{U} \text { of } \mathrm{AK} \text {, Fairbanks, AK, ID \# AF0754 } \\
\text { Jawbone, histological samples to OAD, Anchorage, AK } \\
\text { Jawbone, histological samples transferred from OAD to NIST, NBSB }\end{array}$} \\
\hline
\end{tabular}




\begin{tabular}{|c|c|c|c|c|c|c|}
\hline \multicolumn{7}{|c|}{ BEARDED SEAL (Erignathus barbatus) } \\
\hline $\mathrm{MNnNa}$ & & SAMPLANG & & & LOCATION & LAT /LONG \\
\hline 692-BDSL-001 & 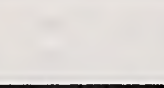 & 26 May 89 & & & $\begin{array}{l}\text { Bering Sea, Norton } \\
\text { Sound, Nome }\end{array}$ & $64^{\circ} 19^{\prime} 165^{\circ} 00^{\prime}$ \\
\hline $\mathrm{SEX}$ & AGE $(y)$ & HENGTH $(\mathrm{cm})$ & WEIGHT $(\mathrm{kg})$ & $\begin{array}{l}\mathrm{FLUKE} \\
\mathrm{wWTH} \\
(\mathrm{cm})\end{array}$ & $\begin{array}{l}\text { AXILARY } \\
\text { GLRTH (cm) }\end{array}$ & THICKNESS (c m) \\
\hline $\mathbf{M}$ & 1 & 166.7 & $68-90$ & & 99 & 4.5 \\
\hline \multirow{2}{*}{ SAMPLES } & \multirow{2}{*}{ NBSSB } & \multicolumn{2}{|c|}{ WEIGHT OF SUBSAMPLES } & & \multirow[t]{2}{*}{ HISTOLOGY } & \multirow{2}{*}{ HOMOGENIZED } \\
\hline & & $A(g)$ & $\mathrm{B}(\mathrm{g})$ & & & \\
\hline Liver & MM3L051 & 154.0 & 163.0 & & yes & 12 Jan 93 \\
\hline Kidney & MM3K052 & 147.0 & 129.5 & & yes & \\
\hline Blubber & MM3B053 & 158.0 & 163.0 & & & \\
\hline \multicolumn{7}{|c|}{ ADDITIONAL SAMPLES COLLECTED, } \\
\hline \multicolumn{7}{|c|}{$\begin{array}{l}\text { Front claws, jawbone, stomach content to } A D F \& G \text {, Nome, AK } \\
\text { Histological samples to OAD, Anchorage, AK }\end{array}$} \\
\hline
\end{tabular}

\begin{tabular}{|c|c|c|c|c|c|c|}
\hline \multicolumn{7}{|c|}{ BEARDED SEAL (Erignathus barbatus) } \\
\hline ANM NO & & SAMPLING & & & LOCATION & LAT/LONG \\
\hline 692-BDSL-002 & & 20 July 89 & & & $\begin{array}{c}\text { Chukchi Sea, } \\
\text { Barrow }\end{array}$ & $71^{\circ} 31^{\prime} 157^{\circ} 00^{\prime}$ \\
\hline $\mathrm{sEX}$ & AGE $(Y)$ & LENGTH $(\mathrm{cm})$ & WEIGHT $(\mathrm{kg})$ & $\begin{array}{l}\mathrm{FU} U \mathrm{KK} \\
\mathrm{wTDTH} \\
\text { (cm) }\end{array}$ & GXILLAR Y & $\begin{array}{l}\text { BLUUBBER } \\
\text { THICKNESS (cm) }\end{array}$ \\
\hline $\mathbf{M}$ & 4 & 210 & 200 & & 130 & 4.5 \\
\hline WBSB & NBSB & \multicolumn{2}{|c|}{ WEIGHT OF SUBSAMPLES } & & HISTOLOGY & \multirow{2}{*}{ HOMOGENIZED } \\
\hline SAMPLES & $\mathrm{IDNO}$ & $\mathrm{A}(\mathrm{g})$ & B (g) & & & \\
\hline Liver & MM3L078 & 149.9 & 157.2 & & yes & \\
\hline Kidney & MM3L079 & 152.6 & 166.9 & & yes & \\
\hline Blubber & MM3B080 & 126.9 & 125.1 & & & \\
\hline \multicolumn{7}{|c|}{ ADDITONAI SANRLES COLIECTED } \\
\hline \multicolumn{7}{|c|}{$\begin{array}{l}\text { Liver, kidney to NMFS, Seattle, WA } \\
\text { Histological samples to OAD, Anchorage, AK }\end{array}$} \\
\hline
\end{tabular}




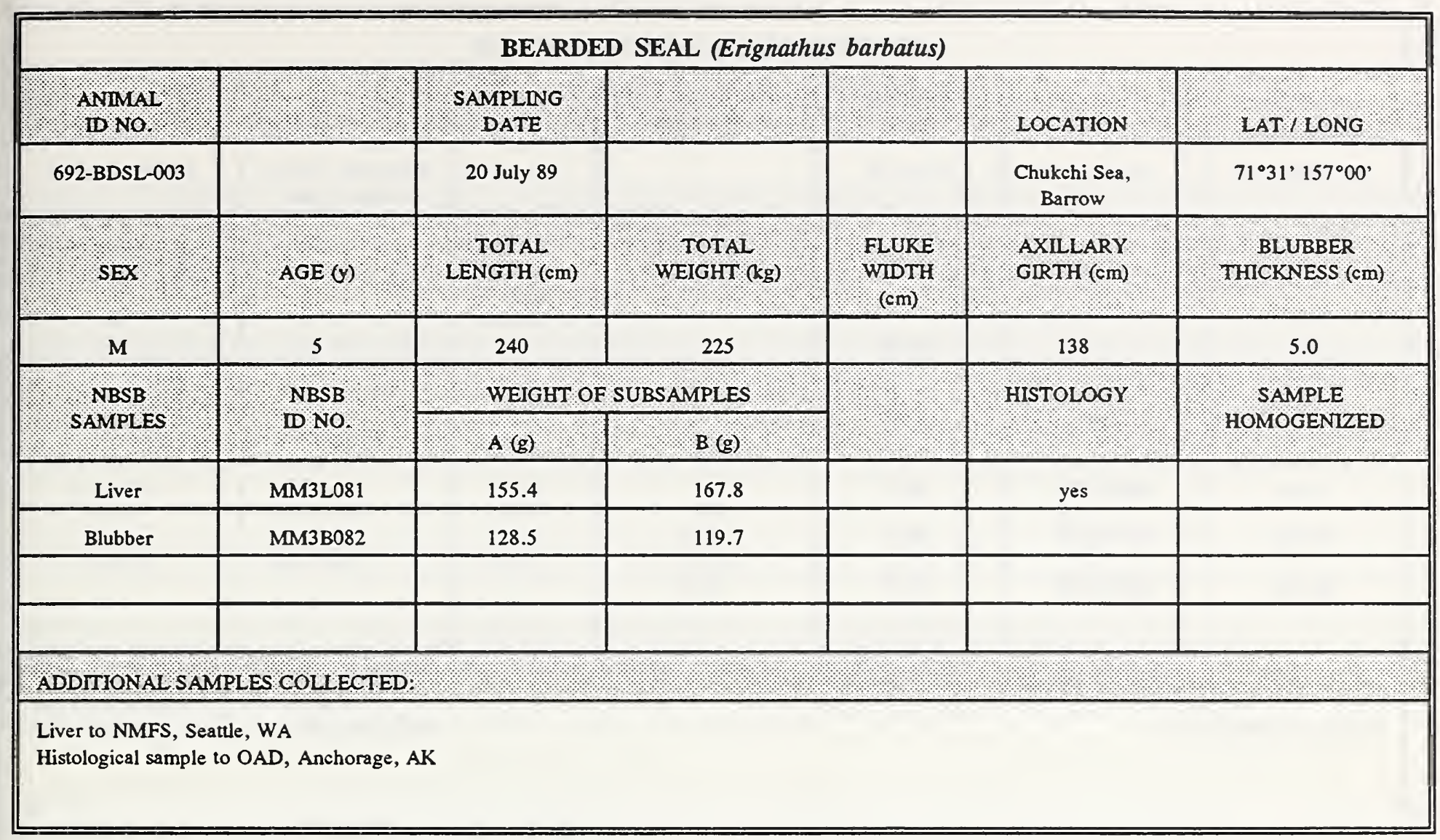

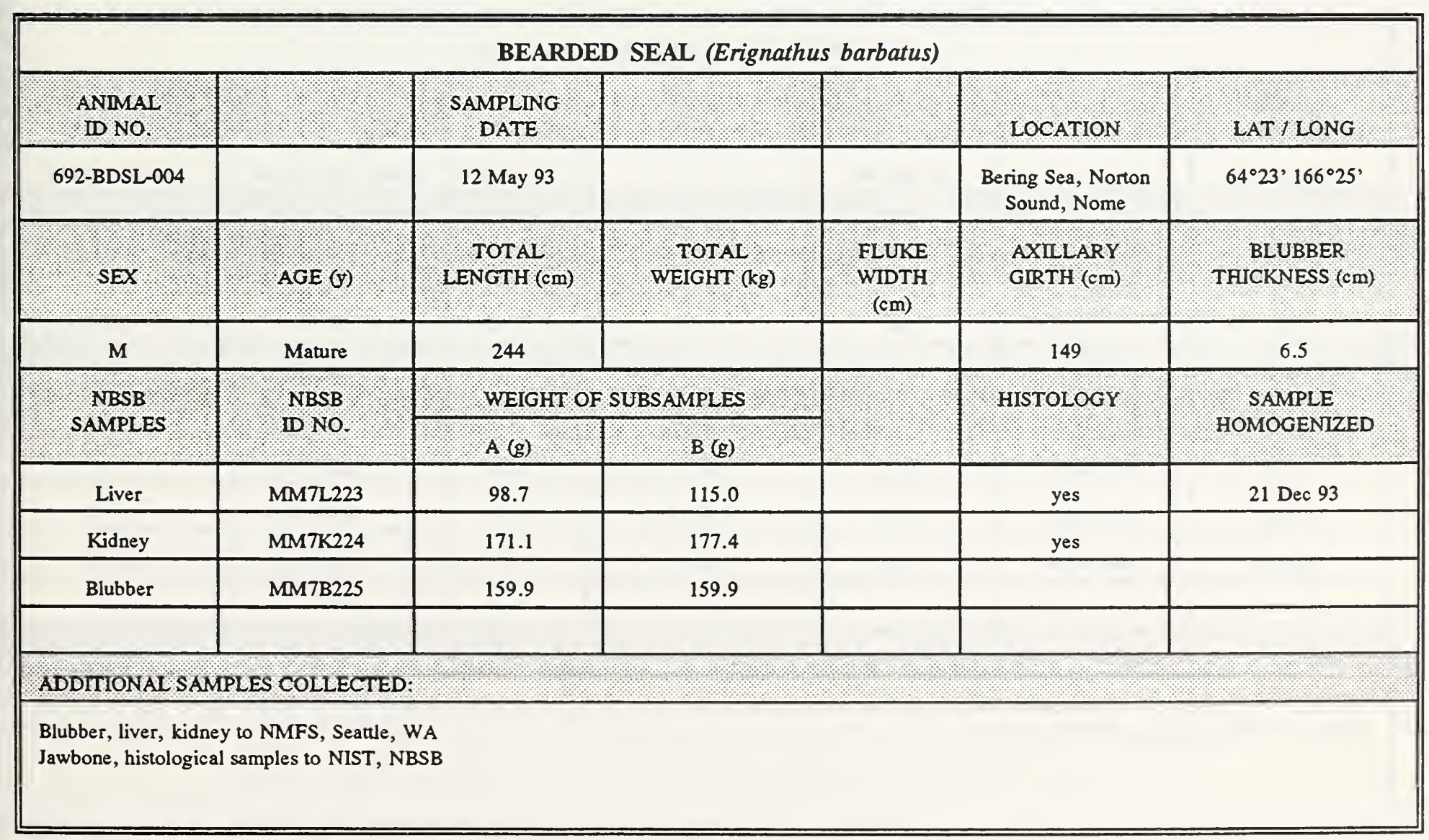




\begin{tabular}{|c|c|c|c|c|c|c|}
\hline \multicolumn{7}{|c|}{ BEARDED SEAL (Erignathus barbatus) } \\
\hline ANimat & & SAMPLING & & & LOCATION & LAT / LONG \\
\hline 692-BDSL-005 & & 3 June 93 & & & $\begin{array}{l}\text { Bering Sea, Norton } \\
\text { Sound, Nome }\end{array}$ & $64^{\circ} 24.5^{\prime} 166^{\circ} 00^{\prime}$ \\
\hline SEX & AGE (U) & $\begin{array}{l}\text { TOTAL } \\
\text { LENGTH (com) }\end{array}$ & WEIGHT (rg) & $\begin{array}{l}\text { FLURE } \\
\text { WIDTH } \\
(\mathrm{cm})\end{array}$ & $\begin{array}{l}\text { AXILLARY } \\
\text { GIRTH (cm) }\end{array}$ & BLUBBER \\
\hline $\mathbf{M}$ & 1 & 161.0 & & & 110 & 4.0 \\
\hline \multirow{2}{*}{ SAMPLES } & \multirow{2}{*}{ ND No. } & \multicolumn{2}{|c|}{ WEIGHT OF SUBSAMPLES } & & \multirow[t]{2}{*}{ Histology } & \multirow{2}{*}{ HOMOGENIZED } \\
\hline & & $\mathrm{A}(\mathrm{g})$ & $\mathrm{B}(\mathrm{g})$ & & & \\
\hline Liver & MM7L250 & 86.7 & & & & $27 \operatorname{Dec} 93$ \\
\hline Kidney & MM7K251 & 69.7 & 84.6 & & & \\
\hline Blubber & MM7B252 & 126.7 & 109.0 & & & \\
\hline \multicolumn{7}{|c|}{ ADDTHONAL SAMPLES COLLECTED. } \\
\hline \multicolumn{7}{|c|}{ Jawbone to NIST, NBSB } \\
\hline
\end{tabular}

\begin{tabular}{|c|c|c|c|c|c|c|}
\hline \multicolumn{7}{|c|}{ BEARDED SEAL (Erignathus barbatus) } \\
\hline $\begin{array}{l}\text { ANIMAL } \\
\text { ID NO. }\end{array}$ & & DATE & & & LOCATION & LAT / LONG \\
\hline 692-BDSL-006 & & 19 May 94 & & & Nome, Cape Nome & $63^{\circ} 59^{\prime} 165^{\circ} 39^{\prime}$ \\
\hline SEX & AGE $(y)$ & LENGTH (cm) & WEIGHT (Kg) & $\begin{array}{l}\text { FLURE } \\
\text { WIDTH } \\
(\mathrm{cm})\end{array}$ & AXRTII (crm) & THICKNESS (oo) \\
\hline $\mathbf{M}$ & & 143 & & & 110 & 3.0 \\
\hline NBSB & NBSB & \multicolumn{2}{|c|}{ WEIGUT OF SUBSAMPLES } & & \multirow[t]{2}{*}{ HISTOLOGY } & \multirow{2}{*}{ HOMOGENIZED } \\
\hline SAMPLES & D NO. & $A(g)$ & B (g) & & & \\
\hline Liver & MM9L264 & 164.7 & 157.9 & & yes & \\
\hline Kidney & MM9K265 & 127.6 & 137.7 & & yes & \\
\hline Blubber & MM9B266 & 152.0 & 152.5 & & & \\
\hline \multicolumn{7}{|c|}{ ADDITIONAL SAMPLES COILECTED } \\
\hline \multicolumn{7}{|c|}{$\begin{array}{l}\text { Blubber, liver, kidney to NWFS, Seattle, WA } \\
\text { Jawbone for teeth, histology to NIST, NBSB }\end{array}$} \\
\hline
\end{tabular}




\begin{tabular}{|c|c|c|c|c|c|c|}
\hline \multicolumn{7}{|c|}{ BEARDED SEAL (Erignathus barbatus) } \\
\hline ANTMAL: & & SAMPLING & & & LOCATION & LAT / LONG \\
\hline 692-BDSL-007 & & 20 May 94 & & & Cape Nome, Nome & $63^{\circ} 59^{\prime} 165^{\circ} 39^{\prime}$ \\
\hline SEX & AGE ( $)$ & $\begin{array}{l}\text { TOTAL } \\
\text { LENGTH }(\mathrm{cm})\end{array}$ & WEIGHT (kg) & $\begin{array}{l}\text { FLUKE } \\
\text { WIDTH } \\
(\mathrm{cm})\end{array}$ & $\begin{array}{l}\text { AXIILARY } \\
\text { GIRTH (cm) }\end{array}$ & $\begin{array}{l}\text { BLUBBER } \\
\text { THICKNESS (cm) }\end{array}$ \\
\hline $\mathbf{M}$ & & 167.6 & & & 114.3 & 3.5 \\
\hline NBSB & NBSB & \multicolumn{2}{|c|}{ WEIGHT OF SUBSAMPLES } & & \multirow[t]{2}{*}{ HISTOLOGY } & \multirow{2}{*}{$\begin{array}{l}\text { SAMPLE } \\
\text { HOMOGENIZED }\end{array}$} \\
\hline SAMPLES & W NO: & $A(g)$ & B (g) & & & \\
\hline Liver & MM9L267 & 149.7 & 135.0 & & yes & \\
\hline Kidney & MM7K268 & 149.7 & 150.1 & & yes & \\
\hline Blubber & MM7B269 & 148.9 & 149.9 & & & \\
\hline \multicolumn{7}{|c|}{ ADDITIONAL SAMPLES COLLECTED; } \\
\hline \multicolumn{7}{|c|}{$\begin{array}{l}\text { Jawbone, histology to NIST, NBSB } \\
\text { Blubber, liver, kidney to NWFS, Seattle, WA }\end{array}$} \\
\hline
\end{tabular}

\begin{tabular}{|c|c|c|c|c|c|c|}
\hline \multicolumn{7}{|c|}{ BEARDED SEAL (Erignathus barbatus) } \\
\hline $\begin{array}{l}\text { ANMMAI } \\
\text { ID NO. }\end{array}$ & & DATE & & & LOCATION & LAT / LONG \\
\hline 692-BDSL-008 & & 22 May 94 & & & Nome, Cape Nome & $63^{\circ} 50^{\prime} 165^{\circ} 39^{\prime}$ \\
\hline SEX & $\operatorname{AGE}(y)$ & LENGTH $(\mathrm{cm})$ & TOTAL & $\begin{array}{l}\text { FLURE } \\
\text { WIDTH } \\
(\mathrm{cm})\end{array}$ & $\begin{array}{l}\text { AXIL LARY } \\
\text { GIRTH (cm) }\end{array}$ & $\begin{array}{l}\text { BLUBBER } \\
\text { THICKNESS }(\mathrm{cm})\end{array}$ \\
\hline $\mathbf{M}$ & & 200.7 & & & 123.2 & 4.5 \\
\hline \multirow{2}{*}{ SAMPLES } & \multirow{2}{*}{ NBSB } & \multicolumn{2}{|c|}{ WEIGHT OF SUBSAMPLES } & & \multirow[t]{2}{*}{ HISTOLOGY } & \multirow{2}{*}{$\begin{array}{l}\text { SAMPLE } \\
\text { HOMOGENIZED }\end{array}$} \\
\hline & & $A(g)$ & $B(g)$ & & & \\
\hline Liver & MM9L270 & 162.6 & 154.9 & & yes & \\
\hline Kidney & MM9K271 & 149.7 & 164.2 & & yes & \\
\hline Blubber & MM9B272 & 167.6 & 156.8 & & & \\
\hline \multicolumn{7}{|c|}{ ADDTTIONAL SAMPLES COLLECTED: } \\
\hline \multicolumn{7}{|c|}{$\begin{array}{l}\text { Jawbone with teeth, histology to NIST, NBSB } \\
\text { Liver, kidney, blubber to NWFS, Seatte, WA }\end{array}$} \\
\hline
\end{tabular}




\begin{tabular}{|c|c|c|c|c|c|c|}
\hline \multicolumn{7}{|c|}{ BEARDED SEAL (Erginathus barbatus) } \\
\hline $\mathrm{ANMMAl}$ & & SAMPL DNG & & & LOCATION & LAT L LONG \\
\hline 692-BDSL-009 & 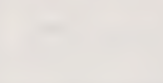 & 23 May 94 & & & $\begin{array}{l}\text { Sludge Island, } \\
\text { Nome }\end{array}$ & $64^{\circ} 19^{\prime} 166^{\circ} 25^{\prime}$ \\
\hline SEX & AGE $(y)$ & LENGTH $(\mathrm{cm})$ & WEIGHT $(\mathrm{kg})$ & $\begin{array}{l}\text { FLUKE } \\
W \mathrm{WITH} \\
(\mathrm{cm})\end{array}$ & AXILARTY & THCKNESS (C.) \\
\hline $\mathbf{F}$ & & 228.6 & & & 144 & 5.7 \\
\hline \multirow{2}{*}{ SAMPLES } & \multirow{2}{*}{ NBSB } & \multicolumn{2}{|c|}{ WEIGHT OF SUBSAMPLES } & & \multirow[t]{2}{*}{ HISTOLOGY } & \multirow{2}{*}{ HOMOGENIZED } \\
\hline & & $A(g)$ & $\mathrm{B}(\mathrm{g})$ & & & \\
\hline Liver & MM9L273 & 173.7 & 179.1 & & yes & \\
\hline Kidney & MM9K274 & 159.8 & 148.2 & & yes & \\
\hline Blubber & MM9B275 & 164.3 & 145.8 & & & \\
\hline \multicolumn{7}{|c|}{ ADDITIONAL S AMPLES COLLECTED. } \\
\hline \multicolumn{7}{|c|}{$\begin{array}{l}\text { Liver, kidney, blubber to NWFS, Seattle, WA } \\
\text { Jawbone, histological samples to NIST, NBSB }\end{array}$} \\
\hline
\end{tabular}




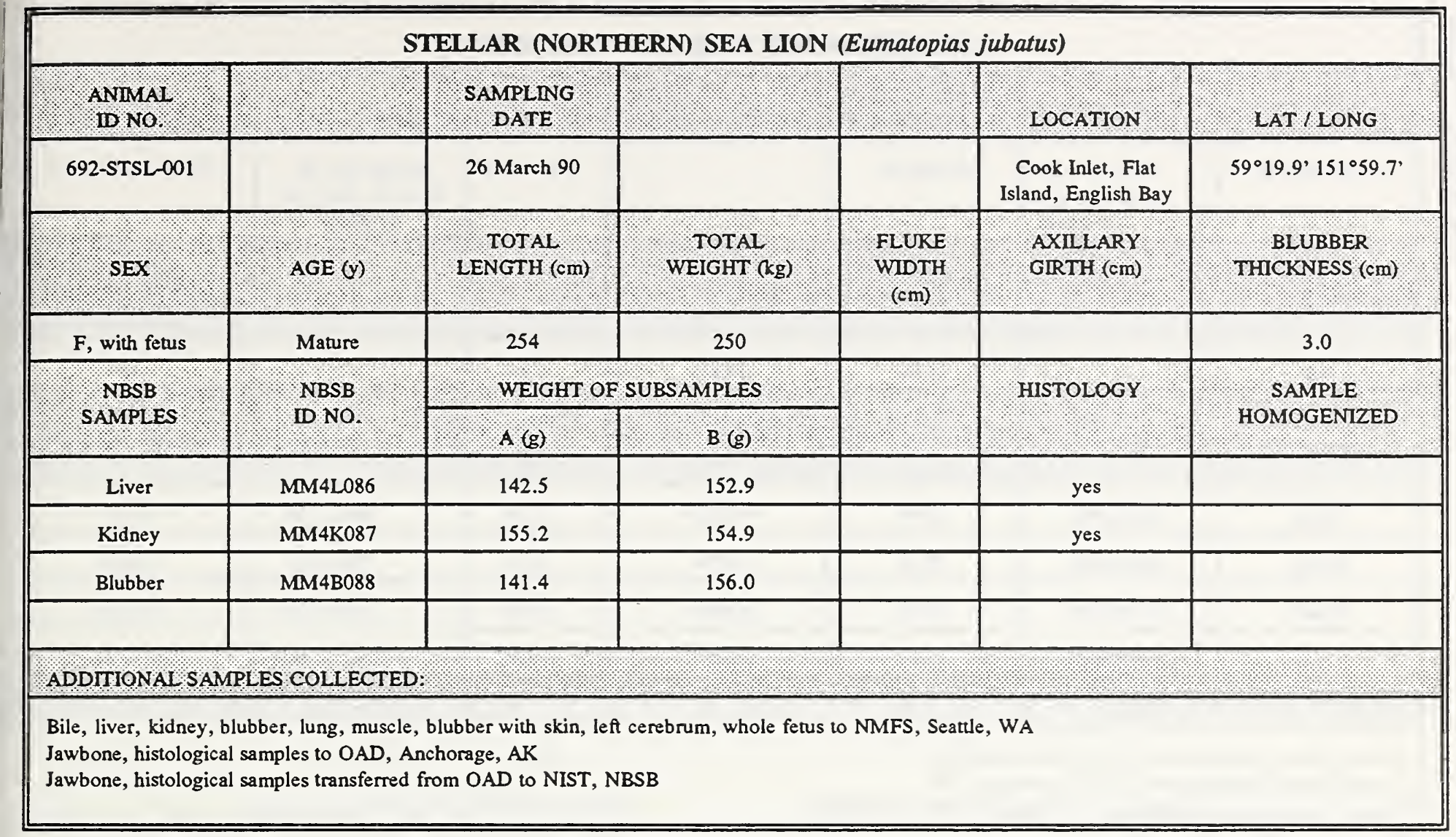




\begin{tabular}{|c|c|c|c|c|c|c|}
\hline \multicolumn{7}{|c|}{ NORTHERN FUR SEAL (Callorhinus ursinus) } \\
\hline ANINALI & & SAMPLING & : & & LOCATION & LAT / LONG \\
\hline 692-FRSL-001 & & 28 July 87 & & & $\begin{array}{l}\text { Bering Sea, St. } \\
\text { Paul IS., Polovina } \\
\text { Rookery }\end{array}$ & $57^{\circ} 10.5^{\prime} 170^{\circ} 09.5^{\prime}$ \\
\hline SEX & AGE (v) & IIENGTH $(\mathrm{cm})$. & WEIOTAT $(\mathrm{r}$. & $\begin{array}{l}\text { FLURE } \\
\text { WIDTH } \\
(\mathrm{cm})\end{array}$ & 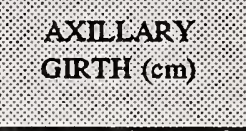 & THICKNESS (cm) \\
\hline $\mathbf{M}$ & 3 & 114.0 & 56.3 & & & 1.5 \\
\hline \multirow{2}{*}{$\begin{array}{l}\text { NBSB } \\
\text { SAMPLES. }\end{array}$} & \multirow{2}{*}{ N N NOB. } & \multicolumn{2}{|c|}{ WEIGHT OF SUBSAMPLES } & & \multirow[t]{2}{*}{ Histologr } & \multirow{2}{*}{ IOMOGENIZED } \\
\hline & & A (g) & $\mathrm{B}(\mathrm{g})$ & & & \\
\hline Liver & MM1L001 & 170.0 & 125.0 & & yes & \\
\hline Kidney & MM1K002 & 93.0 & 97.0 & & yes & \\
\hline Muscle & MM1M003 & 125.0 & 132.5 & & & \\
\hline Blubber & MM1B004 & 114.0 & 114.5 & & & \\
\hline \multicolumn{7}{|c|}{ ADDITIONAL SAMPLES COLLECTED. } \\
\hline \multicolumn{7}{|c|}{$\begin{array}{l}\text { Blood, heart, liver, spleen to NCRC, Frederick, MD } \\
\text { Teeth, histological samples to OAD, Anchorage, AK } \\
\text { Teeth, histological samples transferred from OAD to NIST, NBSB }\end{array}$} \\
\hline
\end{tabular}

\begin{tabular}{|c|c|c|c|c|c|c|}
\hline \multicolumn{7}{|c|}{ NORTHERN FUR SEAL (Callorhinus ursinus) } \\
\hline ANIMAI & & SAMPLING & & & LOCATION & LAT I. LONG \\
\hline 692-FRSL-002 & & 28 July 87 & & & $\begin{array}{l}\text { Bering Sea, St. } \\
\text { Paul Isl., Polovina } \\
\text { Rookery }\end{array}$ & $57^{\circ} 10.5^{\prime} 170^{\circ} 09.5^{\prime}$ \\
\hline SEX & (AGE $(v)$ & I TENGTH $(\mathrm{cm})$. & WEIGHT ( Rg) & WLUKT: & $\begin{array}{l}\text { AXILLARY } \\
\text { GRTH (cm) }\end{array}$ & THICKNESS (cm) \\
\hline $\mathbf{M}$ & 3 & 114.0 & 77.6 & & & 2.5 \\
\hline NBBS & NBSB & \multicolumn{2}{|c|}{ WEIGHT OF SUBSAMTLES. } & & \multirow[t]{2}{*}{ Histoloror } & \multirow{2}{*}{ HOMOGENIZED } \\
\hline SAMPLES & IDNO: & $\alpha(g)$ & $\mathrm{B}(\mathrm{g})$ & & & \\
\hline Liver & MM1L005 & 117.8 & 110.5 & & yes & \\
\hline Kidney & MM1K006 & 137.2 & 137.0 & & yes & \\
\hline Muscle & MM1M007 & 170.0 & 143.0 & & & \\
\hline Blubber & MM1B008 & 156.0 & 158.0 & & & \\
\hline \multicolumn{7}{|c|}{ ADDITIONAY SAMPLES COLLECTED } \\
\hline \multicolumn{7}{|c|}{$\begin{array}{l}\text { Blood, heart, liver, spleen, muscle, skin to NCRC, Frederick, MD } \\
\text { Teeth, histological samples to OAD, Anchorage, AK } \\
\text { Teeth, histological samples transferred from OAD to NIST, NBSB }\end{array}$} \\
\hline
\end{tabular}




\begin{tabular}{|c|c|c|c|c|c|c|}
\hline \multicolumn{7}{|c|}{ NORTHERN FUR SEAL (Callorhinus ursinus) } \\
\hline ANMMAI & & $\begin{array}{l}\text { SAMPLING } \\
\text { DATE }\end{array}$ & & & LOCATION & LAT/LONG \\
\hline 692-FRSL-003 & & 28 July 87 & & & $\begin{array}{l}\text { Bering Sea, St. } \\
\text { Paul Is., Polovina } \\
\text { Rookery }\end{array}$ & $57^{\circ} 10.5^{\prime} 170^{\circ} 09.5^{\prime}$ \\
\hline SEX & AGE $(y)$ & $\begin{array}{l}\text { TENOTAL } \\
\text { LENGTH (cm) }\end{array}$ & $\begin{array}{l}\text { TOTAL : } \\
\text { WEIGHT (kg) }\end{array}$ & $\begin{array}{l}\text { FLURE } \\
\text { WIDTH } \\
.(\mathrm{cm})\end{array}$ & $\begin{array}{l}\text { AXILLARY } \\
\text { GIRTH (cm) }\end{array}$ & $\begin{array}{l}\text { BLUBBER } \\
\text { THICKNESS (cm) }\end{array}$ \\
\hline $\mathbf{M}$ & 3 & 124.0 & 84.0 & & & 3.0 \\
\hline NBSB & NBSB & \multicolumn{2}{|c|}{ WEIGHT OF SUBSAMPLES } & & \multirow[t]{2}{*}{ HISTOLOGY } & \multirow{2}{*}{$\begin{array}{l}\text { SAMPLE } \\
\text { HOMOGENIZED }\end{array}$} \\
\hline SAMPLES & D NO. & A ( $(g)$ & $B(g)$ & & & \\
\hline Liver & MM1L009 & 132.0 & 140.0 & & yes & \\
\hline Kidney & MM1010 & 112.0 & 103.0 & & yes & \\
\hline Muscle & MM1M011 & 152.5 & 136.0 & & & \\
\hline Blubber & MM1B012 & 105.5 & 144.5 & & & \\
\hline \multicolumn{7}{|c|}{ ADDMTIONAL SAMPLES COLLECTED. } \\
\hline \multicolumn{7}{|c|}{$\begin{array}{l}\text { Blood, heart, liver, spleen, skin to NRCR, Frederick, MD } \\
\text { Teeth, histological samples to OAD, Anchorage, AK } \\
\text { Teeth, histological samples transferred from OAD to NIST, NBSB }\end{array}$} \\
\hline
\end{tabular}

\begin{tabular}{|c|c|c|c|c|c|c|}
\hline \multicolumn{7}{|c|}{ NORTHERN FUR SEAL (Callorhinus ursinus) } \\
\hline $\begin{array}{l}\text { ANIMAL } \\
\text { ID NO. }\end{array}$ & & SAMPLING & & & LOCATION & LAT / LONG \\
\hline 692-FRSL-004 & & 29 July 87 & & & $\begin{array}{l}\text { Bering Sea, St. } \\
\text { Paul Is., Northeast } \\
\text { Rookery }\end{array}$ & $57^{\circ} 14.85^{\prime} 170^{\circ} 05.9^{\prime}$ \\
\hline SEX & $A G E(G)$ & IENGTH (cri) & WEIGHT (kg) & $\begin{array}{l}\text { FLUKE } \\
\text { WWTH } \\
(\mathrm{cm})\end{array}$ & $\begin{array}{l}\text { AXILLARY } \\
\text { GIRTH (cm) }\end{array}$ & $\begin{array}{l}\text { BLUBBER } \\
\text { THICKNESS (cm) }\end{array}$ \\
\hline $\mathbf{M}$ & 3 & 111.4 & 64.7 & & & 2.5 \\
\hline \multirow{2}{*}{ SAMBB } & \multirow{2}{*}{ NBSB } & \multicolumn{2}{|c|}{ WEIGHT OF SUBSAMPLES } & & \multirow[t]{2}{*}{ HistoLOGY } & \multirow{2}{*}{$\begin{array}{l}\text { SAMPLE } \\
\text { HOMOGENIZED }\end{array}$} \\
\hline & & $A(g)$ & B (g) & & & \\
\hline Liver & MM1L013 & 129.5 & 103.0 & & yes & 7 Jun 88 \\
\hline Kidney & MM1K014 & 92.0 & 93.5 & & yes & 13 Jun 88 \\
\hline Muscle & MM1M015 & 129.2 & 148.0 & & & 17 Jun 88 \\
\hline Blubber & MM1B016 & 105.5 & 103.0 & & & 13 Jun 88 \\
\hline \multicolumn{7}{|c|}{ ADDITIONAL SAMPLES COLLECTED. } \\
\hline \multicolumn{7}{|c|}{$\begin{array}{l}\text { Blood, heart, spleen, skin to NCRC, Frederick, MD } \\
\text { Teeth, histological samples to OAD, Anchorage, AK } \\
\text { Teeth, histological samples transferred from OAD to NIST, NBSB }\end{array}$} \\
\hline
\end{tabular}




\begin{tabular}{|c|c|c|c|c|c|c|}
\hline \multicolumn{7}{|c|}{ NORTHERN FUR SEAL (Callorhinus ursinus) } \\
\hline annal & & SAMPLNNG & & & LOCATION & LAT / LONG \\
\hline 692-FRSL-005 & & 29 July 87 & & & $\begin{array}{l}\text { Bering Sea, St. } \\
\text { Paul Is., Northeast } \\
\text { Rookery }\end{array}$ & $57^{\circ} 14.85^{\prime} 170^{\circ} 05.9^{\prime}$ \\
\hline $\mathrm{SEX}$ & AGE $(y)$ & leNGTH $(c m)$ & WHIGHA $(\mathrm{Kg})$ & HuKE & GXIRTH (cm) & THICKNESS $(\mathrm{cm})$ \\
\hline $\mathbf{M}$ & 2 & 110.4 & 58.7 & & & 3.0 \\
\hline NBSB & NBSB & \multicolumn{2}{|c|}{ WEIGHT OF SUBSAMPLES } & & HISTOLOGY & \multirow{2}{*}{ HOMOGENIZED } \\
\hline SAMPLES & IDNO. & $A(6)$ & $\mathbf{B}(\mathrm{g})$ & & & \\
\hline Liver & MM1L017 & 145.0 & 136.0 & & yes & 1 Jun 88 \\
\hline Kidney & MM1K018 & 118.5 & 112.0 & & yes & 31 May 88 \\
\hline Muscle & MM1M019 & 103.0 & 105.0 & & & 6 Jun 88 \\
\hline Blubber & $\mathrm{MM} 1 \mathrm{~B} 020$ & 157.0 & 141.0 & & & 26 May 88 \\
\hline \multicolumn{7}{|c|}{ ADDIIONAL SAMPLES COLI ECTED } \\
\hline \multicolumn{7}{|c|}{$\begin{array}{l}\text { Blood, heart, spleen, liver, skin to NCRC, Frederick, MD } \\
\text { Teeth, histological samples to OAD, Anchorage, AK } \\
\text { Teeth, histological samples transferred from OAD to NIST, NBSB }\end{array}$} \\
\hline
\end{tabular}

\begin{tabular}{|c|c|c|c|c|c|c|}
\hline \multicolumn{7}{|c|}{ NORTHERN FUR SEAL (Callorhinus ursinus) } \\
\hline ANMMAI & & SAMPLING & & & LOCATION & IAT/LONG \\
\hline 692-FRSL-006 & & 23 July 90 & & & $\begin{array}{l}\text { Bering Sea, St. } \\
\text { Paul Is., Zapadni } \\
\text { Rookery }\end{array}$ & $57^{\circ} 09^{\prime} 170^{\circ} 20.1^{\prime}$ \\
\hline SEX & AGE ( $)$ & $\begin{array}{l}\text { TOTAL } \\
\text { LENGTH (cm) }\end{array}$ & WEIGHT $(\mathrm{kg})$ & $\mathrm{FU \textrm {UTF }}$ & $\begin{array}{l}\text { A } X I R T H ~ \\
\text { GIR }(\mathrm{cm})\end{array}$ & THICKNESS (cM) \\
\hline $\mathbf{M}$ & $2-3$ & 97.8 & 21.8 & & & 1.2 \\
\hline NBSB & NBSB & \multicolumn{2}{|c|}{ WEIGHT OF SUBSAMPLES } & & histol oGY & \multirow{2}{*}{$\begin{array}{l}\text { SAMPLE } \\
\text { HOMOGENIZED }\end{array}$} \\
\hline SAMPLES & $1 \mathrm{DNO}$ & A (g) & $\mathbf{B}(\mathrm{g})$ & & & \\
\hline Liver & MM4L095 & 138.0 & 166.3 & & yes & \\
\hline Kidney & MM4K096 & 88.0 & 95.3 & & yes & \\
\hline Blubber & MM4B097 & 144.4 & 145.8 & & & \\
\hline \multicolumn{7}{|c|}{ ADDHIONAL SAMPLES COLEECTED } \\
\hline \multicolumn{7}{|c|}{$\begin{array}{l}\text { Blubber, liver to NMFS, Seattle, WA } \\
\text { Jawbone, histological samples to OAD, Anchorage, AK } \\
\text { Jawbone, histological samples transferred from OAD to NIST, NBSB }\end{array}$} \\
\hline
\end{tabular}




\begin{tabular}{|c|c|c|c|c|c|c|}
\hline \multicolumn{7}{|c|}{ NORTHERN FUR SEAL (Callorhinus ursinus) } \\
\hline $\begin{array}{l}\text { ANDMAL } \\
10 \mathrm{NO}\end{array}$ & & SAMPLNNG & & & LOCATION & LAT / LONG \\
\hline 692-FRSL-007 & & 23 July 90 & & & $\begin{array}{l}\text { Bering Sea, St. } \\
\text { Paul Is., Zapadni } \\
\text { Rookery }\end{array}$ & $57^{\circ} 09^{\prime} 170^{\circ} 20.1^{\prime}$ \\
\hline$S E X$ & $A G E(y)$ & LENGTH (cm) & WEIGHT $(\mathrm{kg})$ & $\begin{array}{l}\text { FLUKE } \\
\text { WIDTH } \\
(\mathrm{cm})\end{array}$ & $\begin{array}{l}\text { AXULARY } \\
\text { GIRTH }(\mathrm{cm})\end{array}$ & 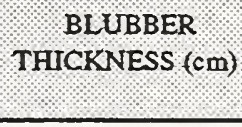 \\
\hline $\mathbf{M}$ & $2-3$ & 103.5 & 23 & & & 0.9 \\
\hline NBSB & NBSB & \multicolumn{2}{|c|}{ WEIGHT OF SUBSAMPLES } & & \multirow[t]{2}{*}{ HISTOLOGY } & \multirow{2}{*}{$\begin{array}{l}\text { SAMPLE } \\
\text { HOMOGENIZED }\end{array}$} \\
\hline SAMPLES & $100 \mathrm{NO}$ & $A(g)$ & $\mathrm{B}(\mathrm{g})$ & & & \\
\hline Liver & MM4L098 & 56.9 & 68.2 & & & 13 Sept 90 \\
\hline Kidney & MM4K099 & 97.5 & 98.6 & & yes & \\
\hline Blubber & MM4B100 & 150.8 & 142.9 & & & \\
\hline \multicolumn{7}{|c|}{ ADDITIONAI SAMPLES COLILCTED. } \\
\hline \multicolumn{7}{|c|}{$\begin{array}{l}\text { Bile, blubber to NMFS, Seattle, WA } \\
\text { Jawbone, histological samples to OAD, Anchorage, AK } \\
\text { Jawbone, histological samples transferred from OAD to NIST, NBSB }\end{array}$} \\
\hline
\end{tabular}

\begin{tabular}{|c|c|c|c|c|c|c|}
\hline \multicolumn{7}{|c|}{ NORTHERN FUR SEAL (Callorhinus ursinus) } \\
\hline $\begin{array}{l}\text { ANDMAI } \\
\text { ID NO. }\end{array}$ & & SAMPLING & & & LOCATION & LAT / LONG \\
\hline 692-FRSL-008 & & 23 July 90 & & & $\begin{array}{l}\text { Bering Sea, St. } \\
\text { Paul Is., Zapadni } \\
\text { Rookery }\end{array}$ & $57^{\circ} 09^{\prime} 170^{\circ} 20.1^{\prime}$ \\
\hline SEX & $A G E(v)$ & LENGTH $(\mathrm{cm})$ & WEIGHT $(\mathrm{kg})$ & $\begin{array}{l}\text { FLUKE } \\
\text { WDTH } \\
\text { (cm) }\end{array}$ & $\begin{array}{l}\text { AXILLARY } \\
\text { GIRTH }(\mathrm{cm})\end{array}$ & $\begin{array}{l}\text { BLUBBER } \\
\text { THICKNESS (cm) }\end{array}$ \\
\hline $\mathbf{M}$ & Subadult & 103.5 & 80.3 & & & 1.1 \\
\hline NBSB & NBSB & \multicolumn{2}{|c|}{ WEIGHT OF SUBSAMPLES } & & \multirow[t]{2}{*}{ HISTOLOGY } & \multirow{2}{*}{$\begin{array}{l}\text { SAMPLE } \\
\text { HOMOGENIZED }\end{array}$} \\
\hline SAMFLES & NON N & $A(g)$ & $\mathrm{B}(\mathrm{g})$ & & & \\
\hline Liver & MM4L101 & 124.2 & 126.0 & & & \\
\hline Kidney & MM4K102 & 81.2 & 80.3 & & yes & \\
\hline Blubber & MM4B103 & 140.0 & 141.7 & & & \\
\hline \multicolumn{7}{|c|}{ ADDTIONAL SAMPLES COLLECTED. } \\
\hline \multicolumn{7}{|c|}{$\begin{array}{l}\text { Bile, blubber, liver to NMFS, Seattle, WA } \\
\text { Jawbone, histological sample to OAD, Anchorage, AK } \\
\text { Jawbone, histological sample transferred from OAD to NIST, NBSB }\end{array}$} \\
\hline
\end{tabular}




\begin{tabular}{|c|c|c|c|c|c|c|}
\hline \multicolumn{7}{|c|}{ NORTHERN FUR SEAL (Callorhinus ursinus) } \\
\hline ANID Nal & & SAMPLNNG & & & LOCATION & LAT / LONG \\
\hline 692-FRSL-009 & & 23 July 90 & & & $\begin{array}{l}\text { Bering Sea, St. } \\
\text { Paul Is., Zapadni } \\
\text { Rookery }\end{array}$ & $57^{\circ} 09^{\prime} 170^{\circ} 20.1^{\prime}$ \\
\hline SEX & $A G E(Y)$ & YENGTH $(\mathrm{cm})$ & WEIGHT $(\mathrm{kg})$ & $\mathrm{HUTH}$ & AXIL ART & THICKNESS (cm) \\
\hline $\mathbf{M}$ & $2-3$ & & & & & \\
\hline WBSB & NBSB & \multicolumn{2}{|c|}{ WEIGHT OF SUBSAMPLES } & & HISTOLOGY & \multirow{2}{*}{ HOMOGENIZED } \\
\hline SAMPLES & ID NO & $A(g)$ & $\mathrm{B}(\mathrm{g})$ & & & \\
\hline Liver & MM4L104 & 137.5 & 144.5 & & yes & \\
\hline Kidney & MM4K105 & 90.2 & 86.1 & & yes & \\
\hline Blubber & MM4B106 & 139.8 & 156.2 & & & \\
\hline \multicolumn{7}{|c|}{ ADDITION AL S AMTLES COLLECTED. } \\
\hline \multicolumn{7}{|c|}{$\begin{array}{l}\text { Bile, blubber, liver to NMFS, Seattle, WA } \\
\text { Jawbone, histological samples to } O A D \text {, Anchorage, AK } \\
\text { Jawbone, histological samples transferred from } O A D \text { to }\end{array}$} \\
\hline
\end{tabular}

\begin{tabular}{|c|c|c|c|c|c|c|}
\hline \multicolumn{7}{|c|}{ NORTHERN FUR SEAL (Callorhinus ursinus) } \\
\hline $\begin{array}{l}\text { ANMMAI } \\
\text { ID NO }\end{array}$ & & SAMPLING & & & LOCATION & IAT $/$ LONG \\
\hline 692-FRSL-010 & & 24 July 90 & & & $\begin{array}{l}\text { Bering Sea, St. } \\
\text { Paul Is., Reef } \\
\text { Rookery }\end{array}$ & $57^{\circ} 09^{\prime} 170^{\circ} 20.1^{\prime}$ \\
\hline SEX & $A G E(y)$ & LENGTH $(\mathrm{cm})$ & WEIGHT $(\mathrm{kg})$ & FLUKE & $\begin{array}{l}\text { AXULARY } \\
\text { GIRTH }(\mathrm{cm})\end{array}$ & THICKNESS (cm) \\
\hline $\mathbf{M}$ & $2-3$ & 94.6 & 15.8 & & & 0.8 \\
\hline רBSB & NBSB & \multicolumn{2}{|c|}{ WEIGHT OF SUBSAMPLES } & & HISTOLOGY & \multirow{2}{*}{ HOMOGENIZED } \\
\hline SAMPLES & DNO & $A(g)$ & $\mathbf{B}(\mathrm{g})$ & & & \\
\hline Liver & MM4L107 & 146.0 & 101.9 & & yes & 18 Sept 90 \\
\hline Kidney & MM4K108 & 66.6 & 68.5 & & yes & \\
\hline Blubber & MM4B109 & 90.0 & 96.7 & & & \\
\hline \multicolumn{7}{|c|}{ ADDITIONAL SAMPLES COLLECTED } \\
\hline \multicolumn{7}{|c|}{$\begin{array}{l}\text { Bile, blubber to NMFS, Seattle, WA } \\
\text { Jawbone, histological samples to OAD, Anchorage, AK } \\
\text { Jawbone, histological samples transferred from OAD to NIST, NBSB }\end{array}$} \\
\hline
\end{tabular}




\begin{tabular}{|c|c|c|c|c|c|c|}
\hline \multicolumn{7}{|c|}{ NORTHERN FUR SEAL (Callorhinus ursinus) } \\
\hline annMal & & SAMPLING & & & LOCATION & LAT / LONG \\
\hline 692-FRSL-011 & & 24 July 90 & & & $\begin{array}{c}\text { Bering Sea, St. } \\
\text { Paul Is., Reef } \\
\text { Rookery }\end{array}$ & $57^{\circ} 06.6^{\prime} 170^{\circ} 17.6^{\prime}$ \\
\hline SEX & AGE $(y)$ & LENGTH (cm) & $\begin{array}{l}\text { TOTAL : } \\
\text { WEIGHT (xg) }\end{array}$ & $\begin{array}{l}\text { FLURE } \\
\text { WIDTH } \\
\text { (cm) }\end{array}$ & $\begin{array}{l}\text { AXILLARY } \\
\text { GIRTH (cm) }\end{array}$ & $\begin{array}{l}\text { BLUBBER } \\
\text { THICKNESS }(\mathrm{cm})\end{array}$ \\
\hline M & $2-3$ & 43.2 & 17.3 & & & 11.0 \\
\hline \multirow{2}{*}{ SAMPBLES } & \multirow{2}{*}{$\begin{array}{l}\text { NBSB } \\
\text { ID NO. }\end{array}$} & \multicolumn{2}{|c|}{ WEIGHT OF SUBSAMPLES } & & \multirow[t]{2}{*}{ HISTOLOGY } & \multirow{2}{*}{, SAMOMLEENIZED } \\
\hline & & $A(g)$ & $\mathrm{B}(\mathrm{g})$ & & & \\
\hline Liver & MOM4L110 & 71.6 & 89.1 & & yes & 21 Sept 90 \\
\hline Kidney & MM4K114 & 70.8 & 90.3 & & yes & \\
\hline Blubber & MM4B112 & 64.1 & 54.4 & & & \\
\hline \multicolumn{7}{|c|}{ ADDITIONAL SAMPLES COLLECTED: } \\
\hline \multicolumn{7}{|c|}{$\begin{array}{l}\text { Bile, blubber to NMFS, Seattle, WA } \\
\text { Jawbone, histological samples to OAD, Anchorage, AK } \\
\text { Jawbone, histological samples transferred from OAD to NIST, NBSB }\end{array}$} \\
\hline
\end{tabular}

\begin{tabular}{|c|c|c|c|c|c|c|}
\hline \multicolumn{7}{|c|}{ NORTHERN FUR SEAL (Callorhinus ursinus) } \\
\hline $\begin{array}{l}\text { ANMMAI } \\
\text { D NO. }\end{array}$ & & SAMPLING & & & LOCATION & LAT / LONG \\
\hline 692-FRSL-012 & & 24 July 90 & & & $\begin{array}{l}\text { Bering Sea, St. } \\
\text { Paul Is., Reef } \\
\text { Rookery }\end{array}$ & $57^{\circ} 06.6^{\prime} 170^{\circ} 17.6^{\prime}$ \\
\hline SEX & AGE ( $)$ & IENGTH (cm) & WEIGHT ( $\mathrm{kg}$ ) & $\begin{array}{l}\text { FLUKE } \\
\text { WTDTH } \\
(\mathrm{cm})\end{array}$ & $\begin{array}{l}\text { AXILARY } \\
\text { GIRTH (cm) }\end{array}$ & $\begin{array}{l}\text { BLUBBER } \\
\text { THICKNESS (cm) }\end{array}$ \\
\hline $\mathbf{M}$ & $2-3$ & 99.1 & 20.2 & & & 1.0 \\
\hline NBSB & NBSB & \multicolumn{2}{|c|}{ WEIGHT OF SUBSAMPLES } & & \multirow[t]{2}{*}{ HISTOLOGY } & \multirow{2}{*}{$\begin{array}{l}\text { SAMPLE } \\
\text { HOMOGENIZED }\end{array}$} \\
\hline SAMPLES & ID NO: & $\mathrm{A}(\mathrm{g})$ & $B(g)$ & & & \\
\hline Liver & MM4L113 & 136.0 & 132.4 & & yes & \\
\hline Kidney & MM4K114 & 70.0 & 90.3 & & yes & \\
\hline Blubber & MM4B115 & 94.4 & 107.6 & & & \\
\hline \multicolumn{7}{|c|}{ ADDITIONAL SAMPLES COLLECTED. } \\
\hline \multicolumn{7}{|c|}{$\begin{array}{l}\text { Bile to NMFS, Seattle, WA } \\
\text { Jawbone, histological samples to OAD, Anchorage, AK } \\
\text { Jawbone, histological samples transferred from OAD to NIST, NBSB }\end{array}$} \\
\hline
\end{tabular}




\begin{tabular}{|c|c|c|c|c|c|c|}
\hline \multicolumn{7}{|c|}{ NORTHERN FUR SEAL (Callorhinus ursinus) } \\
\hline ANMMAL & & SAMPLING & & & LOCATION & LAT LONG \\
\hline 692-FRSL-013 & & 24 July 90 & & & $\begin{array}{l}\text { Bering Sea, St. } \\
\text { Paul Is., Reef } \\
\text { Rookery }\end{array}$ & $57^{\circ} 09^{\prime} 170^{\circ} 20.1^{\prime}$ \\
\hline$S E X$ & $A G E(y)$ & TENGTH $(\mathrm{cm})$ & WEIGHT (lg) & FUTH & $\begin{array}{l}\text { AXII LARY } \\
\text { GIRTH (cm) }\end{array}$ & THICKNESS (cm) \\
\hline $\mathbf{M}$ & $2-3$ & & 20.2 & & & 1.0 \\
\hline NBSB & \multirow{2}{*}{$\begin{array}{l}\mathrm{NBSB} \\
\mathrm{IDNO}\end{array}$} & \multicolumn{2}{|c|}{ WEIGHT OF SUBSAMPLES } & & \multirow[t]{2}{*}{ HISTOLOGY } & \multirow{2}{*}{ HOMOGENIZED } \\
\hline SAMPLES & & $A(g)$ & $\mathrm{B}(\mathrm{g})$ & & & \\
\hline Liver & MM4L116 & 154.1 & 127.0 & & yes & \\
\hline Kidney & MM4K117 & 90.7 & 98.0 & & yes & \\
\hline Blubber & MM4B118 & 129.3 & 98.0 & & & \\
\hline \multicolumn{7}{|c|}{ ADDITIONAI SAMPLES COLLECTED. } \\
\hline \multicolumn{7}{|c|}{$\begin{array}{l}\text { Bile to NMFS, Seattle, WA } \\
\text { Jawbone, histological samples to OAD, Anchorage, AK } \\
\text { Jawbone, histological samples transferred from OAD to }\end{array}$} \\
\hline
\end{tabular}

\begin{tabular}{|c|c|c|c|c|c|c|}
\hline \multicolumn{7}{|c|}{ NORTHERN FUR SEAL (Callorhinus ursinus) } \\
\hline ANnvAr & & SAMPLING & & & LOCATION & LAT / UONG \\
\hline 692-FRSL-014 & & 24 July 90 & & & $\begin{array}{l}\text { Bering Sea, St. } \\
\text { Paul Is., Reef } \\
\text { Rookery }\end{array}$ & $57^{\circ} 06.6^{\prime} 170^{\circ} 17.6^{\prime}$ \\
\hline SEX & $A G E(y)$ & LENGTH $(\mathrm{cm})$ & WEIGHT $(\mathrm{kg})$ & $\begin{array}{l}\text { FLUKE } \\
\text { WWOTH } \\
\text { (cm) }\end{array}$ & $\begin{array}{l}\text { AXILLARY } \\
\text { GIRTH }(\mathrm{cm})\end{array}$ & $\begin{array}{l}\text { BLUBBER } \\
\text { THICKNESS }(\mathrm{cm})\end{array}$ \\
\hline $\mathbf{M}$ & Subadult & 95.9 & 98.0 & & & 0.9 \\
\hline \multirow{2}{*}{ SAMPLES } & \multirow{2}{*}{ NBSB } & \multicolumn{2}{|c|}{ WEIGHT OF SUBSAMPLES } & & \multirow[t]{2}{*}{ HISTOLOGY } & \multirow{2}{*}{ SAMPLE } \\
\hline & & $A(g)$ & $\mathrm{B}(\mathrm{g})$ & & & \\
\hline Liver & MM4L119 & 119.3 & 124.2 & & yes & \\
\hline Kidney & MM4K120 & 92.4 & 98.0 & & yes & \\
\hline Blubber & MM4B121 & 100.0 & 103.7 & & & \\
\hline \multicolumn{7}{|c|}{ ADDITIONA $\mathrm{s}$ ANPLES COL LECTED } \\
\hline \multicolumn{7}{|c|}{$\begin{array}{l}\text { Liver, blubber to NMFS, Seattle, WA } \\
\text { Jawbone, histological samples to OAD, Anchorage, AK } \\
\text { Jawbone, histological samples transferred from OAD to }\end{array}$} \\
\hline
\end{tabular}




\begin{tabular}{|c|c|c|c|c|c|c|}
\hline \multicolumn{7}{|c|}{ PACIFIC WALRUS (Odobenus rosmarus rosmarus) } \\
\hline ID NOF & & SAMPLNG & & & LOCATION & LAT LONG \\
\hline 692-WLRS-001 & & 3 June 93 & & & $\begin{array}{l}\text { Bering Sea, Norton } \\
\text { Sound, Nome }\end{array}$ & $64^{\circ} 05^{\prime} 165^{\circ} 25^{\prime}$ \\
\hline SEX & $\mathrm{AGE}(y)$ & LENGTH $(\mathrm{cm})$ & WEIGHT $(\mathrm{kg})$ & $\begin{array}{l}\text { FLUKE } \\
\text { WIDTH } \\
(\mathrm{cm})\end{array}$ & AXIL LARY & THICKNESS (CM) \\
\hline $\mathbf{M}$ & $12-15$ & & & & & 2.5 \\
\hline NBBS & \multirow{2}{*}{ NBSB } & \multicolumn{2}{|c|}{ WEIGHT OF SUBSAMPLES } & & HISTOLOGY & \multirow{2}{*}{ HOMOGENIZED } \\
\hline SAMPLES & & $A(g)$ & $\mathrm{B}(\mathrm{g})$ & & & \\
\hline Liver & MM7L253 & 113.7 & 128.3 & & & \\
\hline Kidmey & MM7K254 & 124.3 & 85.4 & & & \\
\hline Blubber & MM7B255 & 95.6 & 105.5 & & & \\
\hline \multicolumn{7}{|c|}{ ADDITIONAL SAMPLES COLLECTED. } \\
\hline \multicolumn{7}{|c|}{$\begin{array}{l}2 \text { Teeth to NIST, NBSB } \\
1 \text { Tooth for aging transferred from NIST, NBSB to U.S. Fish \& Wildlife Service, Anchorage, AK }\end{array}$} \\
\hline
\end{tabular}

\begin{tabular}{|c|c|c|c|c|c|c|}
\hline \multicolumn{7}{|c|}{ PACIFIC WALRUS (Odobenus rosmarus rosmarus) } \\
\hline $\begin{array}{l}\text { ANMMAI } \\
\mathrm{DNO}\end{array}$ & & SAMPLING & & & LOCATION & LAT L LONG \\
\hline 692-WRLS-002 & & 3 June 93 & & & $\begin{array}{l}\text { Bering Sea, Norton } \\
\text { Sound, Nome }\end{array}$ & $64^{\circ} 15^{\prime} 165^{\circ} 25^{\prime}$ \\
\hline $\mathrm{SEX}$ & $\mathrm{AGE}(\mathrm{Y})$ & TENGTH (cm) & WEIGHT $(\mathrm{kg})$ & $\begin{array}{l}\mathrm{FLUK} \\
\mathrm{WIDTH} \\
(\mathrm{cm})\end{array}$ & GLTLL AR Y & THICKNESS (cm) \\
\hline $\mathbf{M}$ & $8-10$ & & & & & 2.5 \\
\hline NBSB & $\mathrm{NBSB}$ & \multicolumn{2}{|c|}{ WEIGHT OF SUBSAMPLES } & & HISTOLOGY & SAMNLE \\
\hline SAMPLES & $10 \mathrm{NO}$ & $A(g)$ & B (g) & & & HOMOGENLAED \\
\hline Liver & MM7L256 & 115.9 & 84.3 & & & \\
\hline Kidney & MM7K257 & 150.4 & 169.6 & & & \\
\hline Blubber & MM7B258 & 103.1 & 95.7 & & & \\
\hline \multicolumn{7}{|c|}{ ADDITONAL SAMPLES COLLECTED } \\
\hline \multicolumn{7}{|c|}{$\begin{array}{l}3 \text { Teeth to NIST, NBSB } \\
1 \text { Tooth for aging transferred from NIST, NBSB to U.S. Fish \& Wildlife Service, Anchorage, AK }\end{array}$} \\
\hline
\end{tabular}




\begin{tabular}{|c|c|c|c|c|c|c|}
\hline \multicolumn{7}{|c|}{ PACIFIC WALRUS (Odobenus rosmarus rosmarus) } \\
\hline $\begin{array}{l}\text { ANMMAL } \\
10 \text { NO. }\end{array}$ & & $\begin{array}{l}\text { SAMPLING } \\
\text { DATE }\end{array}$ & & & LOCATION & LAT / LONG \\
\hline 692-WLRS-003 & & 30 May 94 & & & Nome & $64^{\circ} 14^{\prime} 165^{\circ} 25^{\prime}$ \\
\hline SEX & AGE (V) & $\begin{array}{l}\text { TOTAL } \\
\text { LENGTH (cm) }\end{array}$ & WEIGHT $(\mathrm{kg})$ & $\begin{array}{l}\text { FLUKE } \\
\text { WIDTH } \\
(\mathrm{cm})\end{array}$ & $\begin{array}{l}\text { AXILLARY } \\
\text { GIRTH (cm) }\end{array}$ & $\begin{array}{l}\text { BLUBBER } \\
\text { THICKNESS (cm) }\end{array}$ \\
\hline $\mathbf{M}$ & & 284 & & 52 & 300 & 5.0 \\
\hline NBSB & NBSB & \multicolumn{2}{|c|}{ WEIGHT OF SUBSAMPLES } & & \multirow[t]{2}{*}{ HISTOLOGY } & \multirow{2}{*}{$\begin{array}{l}\text { SAMPLE } \\
\text { HOMOGENIZED }\end{array}$} \\
\hline SAMPLES & ID NO. & $A(g)$ & $B(g)$ & & & \\
\hline Liver & MM9L298 & 166.1 & 169.5 & & yes & \\
\hline Kidney & MM9K299 & 163.8 & 166.1 & & yes & \\
\hline Blubber & MM9B300 & 167.3 & 166.3 & & yes & \\
\hline \multicolumn{7}{|c|}{ ADDTIONAL SAMPLES COLLECTED: } \\
\hline \multicolumn{7}{|c|}{$\begin{array}{l}2 \text { Teeth } \\
\text { Histology to NIST, NBSB }\end{array}$} \\
\hline
\end{tabular}

\begin{tabular}{|c|c|c|c|c|c|c|}
\hline \multicolumn{7}{|c|}{ PACIFIC WALRUS (Odobenus rosmarus rosmarus) } \\
\hline $\begin{array}{l}\text { ANDMAL } \\
\text { ID NO. }\end{array}$ & & $\begin{array}{l}\text { SAMPLING } \\
\text { DATE }\end{array}$ & & & LOCATION & LAT / LONG \\
\hline 692-WRLS-004 & & 30 May 94 & & & Nome & $64^{\circ} 14^{\prime} 165^{\circ} 25^{\prime}$ \\
\hline SEX & AGE $(r)$ & $\begin{array}{l}\text { TENGTH } \\
\text { LEN (cm) }\end{array}$ & WEIGHT $(\mathrm{kg})$. & $\begin{array}{l}\text { FLUKE } \\
\text { WIDTH } \\
\text { (cm) }\end{array}$ & $\begin{array}{l}\text { AXILLARY } \\
\text { GIRTH (cm) }\end{array}$ & $\begin{array}{l}\text { BLUBBER } \\
\text { THICKNESS (cm) }\end{array}$ \\
\hline $\mathbf{M}$ & & 312 & & 55 & & 3.25 \\
\hline \multirow{2}{*}{ SAMPLES } & \multirow{2}{*}{ NBSB } & \multicolumn{2}{|c|}{ WEIGHT OF SUBSAMPLES } & & \multirow[t]{2}{*}{ HISTOLOGY } & \multirow{2}{*}{$\begin{array}{l}\text { SAMPLE } \\
\text { HOMOGENIZED }\end{array}$} \\
\hline & & $A(g)$ & B (g) & & & \\
\hline Liver & MM9L301 & 170.4 & 140.0 & & yes & \\
\hline Kidney & MM9K302 & 166.0 & 150.9 & & yes & \\
\hline Blubber & MM9B303 & 153.2 & 158.9 & & & \\
\hline \multicolumn{7}{|c|}{ ADDITIONAL SAMPLES COLLECTED: } \\
\hline \multicolumn{7}{|c|}{$\begin{array}{l}2 \text { Teeth } \\
\text { Histology to NIST, NBSB }\end{array}$} \\
\hline
\end{tabular}




\begin{tabular}{|c|c|c|c|c|c|c|}
\hline \multicolumn{7}{|c|}{ BELUGA WHALE (Delphinapterus leucas) } \\
\hline $\begin{array}{l}\text { ANDMAL } \\
\text { ID NO. }\end{array}$ & & $\begin{array}{l}\text { SAMPLING } \\
\text { DATE }\end{array}$ & & & LOCATION & LAT / LONG \\
\hline 692-BLKA-001 & & 26 May 89 & & & $\begin{array}{c}\text { Chukchi Sea, Point } \\
\text { Hope }\end{array}$ & $68^{\circ} 20^{\prime} 166^{\circ} 50^{\prime}$ \\
\hline SEX & AGE $(y)$ & $\begin{array}{l}\text { TOTAL } \\
\text { LENGTH }(\mathrm{cm})\end{array}$ & $\begin{array}{l}\text { TOTAL } \\
\text { WEIGHT }(\mathrm{kg})\end{array}$ & $\begin{array}{l}\text { FLURE } \\
\text { WIDTH } \\
\text { (cm) }\end{array}$ & $\begin{array}{l}\text { AXILLARY } \\
\text { GIRTH (cm) }\end{array}$ & $\begin{array}{l}\text { BLUBBER } \\
\text { THICKNESS }(\mathrm{cm})\end{array}$ \\
\hline $\mathbf{F}$ & $16^{*}$ & 342.9 & & 74.9 & & \\
\hline \multirow{2}{*}{$\begin{array}{l}\text { NBSB } \\
\text { SAMPLES }\end{array}$} & \multirow{2}{*}{$\begin{array}{l}\text { NBSB } \\
\text { ID NO }\end{array}$} & \multicolumn{2}{|c|}{ WEIGHT OF SUBSAMPLES } & & \multirow[t]{2}{*}{ HISTOLOGY } & \multirow{2}{*}{$\begin{array}{l}\text { SAMPLE } \\
\text { HOMOGENIZED }\end{array}$} \\
\hline & & $A(g)$ & $B(g)$ & & & \\
\hline Liver & MM3L069 & 178.6 & 166.6 & & yes & 12 Oct 90 \\
\hline Kidney & MM3K070 & 148.0 & 169.0 & & yes & \\
\hline Blubber & MM3B071 & 121.9 & 132.5 & & & 24 Sept 90 \\
\hline \multicolumn{7}{|c|}{ ADDTIONAL SAMPLES COLLECTED: } \\
\hline \multicolumn{7}{|c|}{$\begin{array}{l}\text { *Age is based on the number of growth rings counted in teeth, not an absolute age. Age determined by Robert Suydam. } \\
\text { Lower jaw with teeth, blubber, liver, reproductive organs, kidney, skin to NBS DWM, Barrow, AK ID \# HDL-5-89 } \\
\text { Histological samples to OAD, Anchorage, AK } \\
\text { Histological samples trasferred from OAD to NIST, NBSB }\end{array}$} \\
\hline
\end{tabular}

\begin{tabular}{|c|c|c|c|c|c|c|}
\hline \multicolumn{7}{|c|}{ BELUGA WHALE (Delphinapterus leucas) } \\
\hline $\begin{array}{l}\text { ANDMAL } \\
\text { ID NO. }\end{array}$ & & $\begin{array}{l}\text { SAIMPLING } \\
\text { DATE }\end{array}$ & & & LOCATION & LAT / LONG \\
\hline 692-BLKA-002 & & 25 May 89 & & & $\begin{array}{c}\text { Chukchi Sea, Point } \\
\text { Hope }\end{array}$ & $68^{\circ} 20^{\prime} 166^{\circ} 50^{\prime}$ \\
\hline SEX & AGE $(\theta)$ & LENGTH $(\mathrm{cm})$ & WEIGHT $(\mathrm{kg})$ & $\begin{array}{l}\text { FLUKE } \\
\text { WIDTH } \\
\text { (cm) }\end{array}$ & $\begin{array}{l}\text { AXILLARY } \\
\text { GIRTH }(\mathrm{cm})\end{array}$ & $\begin{array}{c}\text { BLUBBER } \\
\text { THICKNESS }(\mathrm{cm})\end{array}$ \\
\hline $\mathrm{F}$ & $9 *$ & 309.9 & & 71.1 & & 7.6 \\
\hline NBSB & NBSB & \multicolumn{2}{|c|}{ WEIGHT OF SUBSAMPLES } & & \multirow[t]{2}{*}{ HISTOLOGY } & \multirow{2}{*}{$\begin{array}{l}\text { SAMIPLE } \\
\text { HOMOGENIZED }\end{array}$} \\
\hline SAMPLES & ID NO. & $\mathrm{A}(\mathrm{g})$ & $\mathrm{B}(\mathrm{g})$ & & & \\
\hline Liver & MM3L072 & 178.3 & 166.6 & & yes & 3 May 90 \\
\hline Kidney & MM3K073 & 155.6 & 163.1 & & yes & \\
\hline Blubber & $\mathrm{MM} 3 \mathrm{~B} 074$ & 129.9 & 132.0 & & & 25 Sept 90 \\
\hline \multicolumn{7}{|c|}{ ADDRIONAI SAMIPLES COLLECTED. } \\
\hline \multicolumn{7}{|c|}{$\begin{array}{l}\text { *Age is based on the number of growth rings counted in teeth, not an absolute age. Age determined by Robert Suydam. } \\
\text { Lower jaw with teeth, blubber, liver, reproductive organs, kidney, skin to NSB DWM, Barrow, AK ID \# HDL-3-89 } \\
\text { Histological samples to OAD, Anchorage, AK } \\
\text { Histological samples transferred from OAD to NIST, NBSB }\end{array}$} \\
\hline
\end{tabular}




\begin{tabular}{|c|c|c|c|c|c|c|}
\hline \multicolumn{7}{|c|}{ BELUGA WHALE (Delphinapterus leucas) } \\
\hline ANDMAL & & SAMPLNG & & & LOCATION & LAT 1 LONG \\
\hline 692-BLKA-003 & & 25 May 89 & & & $\begin{array}{c}\text { Chukchi Sea, Point } \\
\text { Hope }\end{array}$ & $68^{\circ} 20^{\prime} 166^{\circ} 50^{\prime}$ \\
\hline $\mathrm{SEX}$ & $\mathrm{AGE}(y)$ & LENGTH (cm) & WEIGHT $(\mathrm{kg})$ & FLUKE & $\begin{array}{l}\text { AXILLARY } \\
\text { GIRTH }(\mathrm{cm})\end{array}$ & THICKNESS (cm) \\
\hline F & $20 *$ & 348 & & & & \\
\hline \multirow{2}{*}{ SAMPSB } & \multirow{2}{*}{$\begin{array}{l}\mathrm{NBSB} \\
\mathrm{DNO}\end{array}$} & \multicolumn{2}{|c|}{ WEIGHT OF SUBSAMPLES } & & \multirow[t]{2}{*}{ HISTOLOGY } & \multirow{2}{*}{ HOMOGENIZED } \\
\hline & & $A(g)$ & $\mathrm{B}(\mathrm{g})$ & & & \\
\hline Liver & MM3L075 & 156.1 & 170.1 & & yes & 3 May 90 \\
\hline Kidney & MM3K076 & 176.3 & 175.0 & & yes & \\
\hline & & & & & & \\
\hline & & & & & & \\
\hline \multicolumn{7}{|c|}{ ADDITIONALSAMPLES COLLECTED. } \\
\hline \multicolumn{7}{|c|}{$\begin{array}{l}\text { *Age is based on the number of growth rings counted in teeth, not an absolute age. Age determined by } \\
\text { Lower jaw with teeth, liver, reproductive organs, kidney to NBS DWM, Barrow, AK, ID \# HDL-2-89 } \\
\text { Histological samples to OAD, Anchorage, AK } \\
\text { Histological samples transferred from OAD to NIST, NBSB }\end{array}$} \\
\hline
\end{tabular}

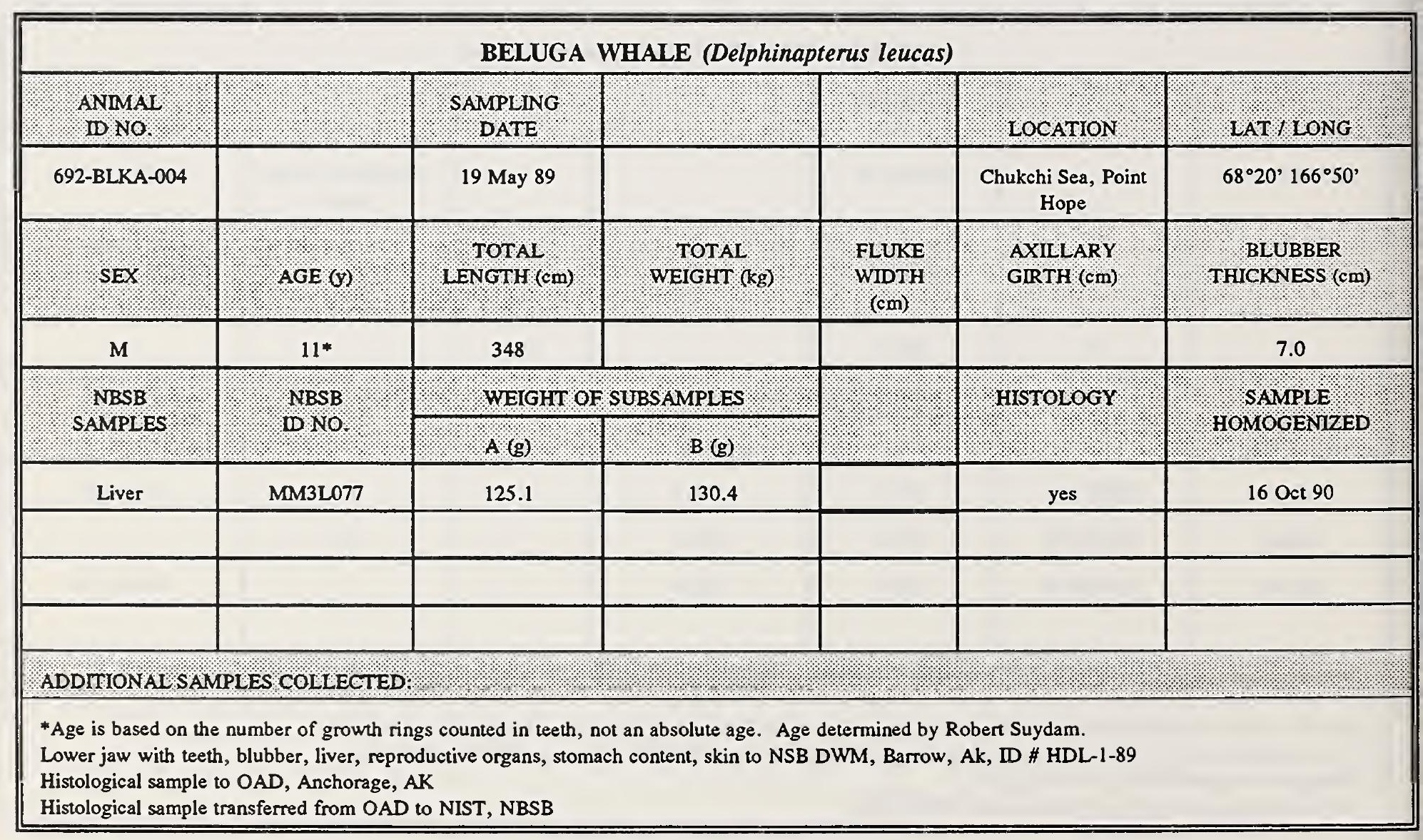




\begin{tabular}{|c|c|c|c|c|c|c|}
\hline \multicolumn{7}{|c|}{ BELUGA WHALE (Delphinapterus leucas) } \\
\hline ANMMAL & & $\begin{array}{l}\text { SAMPLING } \\
\text { DATE }\end{array}$ & & & LOCATION & LAT / LONG \\
\hline 692-BLKA-005 & & 11 July 90 & & & $\begin{array}{c}\text { Chukchi Sea, Point } \\
\text { Lay }\end{array}$ & $69^{\circ} 05163^{\circ} 45^{\prime}$ \\
\hline SEX & $A G E(y)$ & $\begin{array}{l}\text { TETAL } \\
\text { LENGTH (cm) }\end{array}$ & $\begin{array}{l}\text { TOTAL } \\
\text { WEIGHT ( } \mathrm{sg} \text { ) }\end{array}$ & $\begin{array}{l}\text { FLURE } \\
\text { WIDTH } \\
(\mathrm{cm})\end{array}$ & $\begin{array}{l}\text { AXILLARY } \\
\text { GIRTH (cm) }\end{array}$ & THMCKNESS (cm) \\
\hline $\mathbf{M}$ & $30 *$ & 394 & & 103 & & 3.7 \\
\hline NBSB & $\mathrm{NBSB}$ & \multicolumn{2}{|c|}{ WEIGHT OF SUBSAMPLES } & & \multirow[t]{2}{*}{ HistoloGY } & \multirow{2}{*}{ HOMOGENIZED } \\
\hline SAMPLES & ID NO: & $A(g)$ & $\mathrm{B}(\mathrm{g})$ & & & \\
\hline Liver & MM4L125 & 175.75 & 169.6 & & yes & 26 Aug 92 \\
\hline Kidney & MM4K126 & 169.2 & 160.85 & & yes & \\
\hline Blubber & MM4B127 & 131.1 & 131.85 & & & $8 \operatorname{Jan} 91$ \\
\hline \multicolumn{7}{|c|}{ ADDIIONAL SAMPLES COLLECTED: } \\
\hline \multicolumn{7}{|c|}{$\begin{array}{l}\text { *Age is based on the number of growth rings counted in teeth, not an absolute age. Age determined by Robert Suydam. } \\
\text { Teeth, skin to NBS DWM, Barrow, AK, ID \# LDL-2-90 } \\
\text { Teeth, histological samples to OAD, Anchorage, AK } \\
\text { Teeth, histological samples transferred from OAD to NIST, NBSB }\end{array}$} \\
\hline
\end{tabular}

\begin{tabular}{|c|c|c|c|c|c|c|}
\hline \multicolumn{7}{|c|}{ BELUGA WHALE (Delphinapterus leucas) } \\
\hline $\begin{array}{l}\text { ANIMAI } \\
\text { ID NO. }\end{array}$ & & $\begin{array}{l}\text { SAMPLING } \\
\text { DATE }\end{array}$ & & & LOCATION & LAT / LONG \\
\hline 692-BLKA-006 & & 11 July 90 & & & $\begin{array}{c}\text { Chukchi Sea, Point } \\
\text { Lay }\end{array}$ & $69^{\circ} 05^{\prime} 163^{\circ} 45^{\prime}$ \\
\hline SEX & AGE (y) & $\begin{array}{l}\text { TENAL } \\
\text { LENGTH (cm) }\end{array}$ & $\begin{array}{l}\text { TOTAL } \\
\text { WEIGHT (kg) }\end{array}$ & $\begin{array}{l}\text { FLUKE } \\
\text { WIDTH } \\
(\mathrm{cm})\end{array}$ & $\begin{array}{l}\text { AXILLARY } \\
\text { GIRTH (cm) }\end{array}$ & $\begin{array}{l}\text { BLUBBER } \\
\text { THICKNESS }(\mathrm{cm})\end{array}$ \\
\hline M & $\geq 5$ & 430 & & 102 & & 6.5 \\
\hline NBSB : : & NBSB & \multicolumn{2}{|c|}{ WEIGHT OF SUBSAMPLES. } & & \multirow[t]{2}{*}{ HISTOLOGY } & \multirow{2}{*}{ SAMPLE } \\
\hline SAMFLES & & $A(g)$ & B (g) & & & \\
\hline Liver & MM4L128 & 184.9 & 180.8 & & yes & 27 Aug 92 \\
\hline Kidney & MM4K129 & 154.65 & 176.75 & & yes & \\
\hline Blubber & MM4B130 & 152.8 & 146.2 & & & $9 \operatorname{Jan} 91$ \\
\hline \multicolumn{7}{|c|}{ ADDITIONAL SAMPLES COLLECTED. } \\
\hline \multicolumn{7}{|c|}{$\begin{array}{l}\text { Teeth to NSB DWM, Barrow, AK, ID \# LDL-31-90 } \\
\text { Histological samples to OAD, Anchorage, AK } \\
\text { Histological samples transferred from OAD to NIST, NBSB }\end{array}$} \\
\hline
\end{tabular}




\begin{tabular}{|c|c|c|c|c|c|c|}
\hline \multicolumn{7}{|c|}{ BELUGA WHALE (Delphinapterus leucas) } \\
\hline ANamai & & SAMPLDNG & & & LOCATION & LAT / LONG \\
\hline 692-BLKA-007 & & 11 July 90 & & & $\begin{array}{l}\text { Chukchi Sea, Point } \\
\text { Lay }\end{array}$ & $69^{\circ} 05163^{\circ} 45^{\prime}$ \\
\hline $\mathrm{SEX}$ & AGE (y) & LENGTH $(\mathrm{cm})$ & WEIGHT $(\mathrm{gg})$ & $\begin{array}{l}\text { FLUKE } \\
\text { WIDTH } \\
\text { (cm) }\end{array}$ & $\begin{array}{l}\text { AXILLARY } \\
\text { GIRTH }(\mathrm{cm})\end{array}$ & THICKNESS (cm) \\
\hline $\mathbf{F}$ & $43 *$ & 363 & & 88 & & 7.7 \\
\hline NBSB & \multirow{2}{*}{ NBNSB } & \multicolumn{2}{|c|}{ WEIGHT OF SUBSAMPLES } & & \multirow[t]{2}{*}{ HISTOLOGY } & \multirow{2}{*}{ HOMOGENIZED } \\
\hline SAMPLES & & $8(\mathrm{~g})$ & $\mathrm{B}(\mathrm{g})$ & & & \\
\hline Liver & MM4L134 & 174.5 & 158.25 & & yes & 19 Oct 90 \\
\hline Kidney & MM4K132 & 159.75 & 159.8 & & yes & \\
\hline Blubber & MM4B133 & 140.9 & 137.2 & & & 28 Sept 90 \\
\hline \multicolumn{7}{|c|}{ ADDITIONAI SAMPLES COLLECTED. } \\
\hline \multicolumn{7}{|c|}{$\begin{array}{l}\text { *Age is based on the number of growth rings counted in teeth, not an } \\
\text { Teeth, reproductive organs to NSB DWM, Barrow, AK, ID \# LDL-1 } \\
\text { Teeth, histological samples to OAD, Anchorage, AK } \\
\text { Teeth, histological samples transferred from OAD to NIST, NBSB }\end{array}$} \\
\hline
\end{tabular}

\begin{tabular}{|c|c|c|c|c|c|c|}
\hline \multicolumn{7}{|c|}{ BELUGA WHALE (Delphinapterus leucas) } \\
\hline ANnMAI & & SAMPLING & & & LOCATION & LAT / LONG \\
\hline 692-BLKA-008 & & 11 July 90 & & & $\begin{array}{c}\text { Chukchi Sea, Point } \\
\text { Lay }\end{array}$ & $69^{\circ} 05^{\prime} 163^{\circ} 45^{\prime}$ \\
\hline SEX & AGE (Y) & LENGTH $(\mathrm{cm})$ & WEIGHT $(\mathrm{kg})$ & FLUKE & $\begin{array}{l}\text { AXIL LAR } \\
\text { GLRTH }\left(\mathrm{cm}_{\mathrm{r}}\right)\end{array}$ & THICKNESS $(\mathrm{cm})$ \\
\hline $\mathbf{M}$ & $13^{*}$ & 364 & & & & \\
\hline \multirow{2}{*}{ SAMPLE } & \multirow{2}{*}{$\begin{array}{l}\mathrm{NBSB} \\
\mathrm{1DNO}\end{array}$} & \multicolumn{2}{|c|}{ WEIGHT OF SUBSAMPLES } & & HISTOLOGY & \multirow{2}{*}{ HOMOGENIZED } \\
\hline & & ( $(\mathrm{g})$ & B (g) & & & \\
\hline Liver & MM4L134 & 172.0 & 160.15 & & yes & 3 Sept 92 \\
\hline Kidney & MM4K135 & 149.6 & 139.25 & & yes & \\
\hline Blubber & MM4B136 & 130.0 & 135.9 & & & $16 \operatorname{Jan} 91$ \\
\hline \multicolumn{7}{|c|}{ ADDMIONAL SAMPLES COLLECTED. } \\
\hline \multicolumn{7}{|c|}{$\begin{array}{l}\text { *Age is based on the number of growth rings counted in teeth, not } \\
\text { Teeth to NSB DWM, Barrow, AK, ID \# LDL-62-90 } \\
\text { Teeth, histological samples to OAD, Anchorage, AK } \\
\text { Teeth, histological samples transferred srom OAD to NIST, NBSB }\end{array}$} \\
\hline
\end{tabular}




\begin{tabular}{|c|c|c|c|c|c|c|}
\hline \multicolumn{7}{|c|}{ BELUGA WHALE (Delphinapterus leucas) } \\
\hline AnIMAL & & SAMPLING & & & LOCATION & LAT / LONG \\
\hline 692-BLKA-009 & & 11 July 90 & & & $\begin{array}{l}\text { Chukchi Sea, Point } \\
\text { Lay }\end{array}$ & $69^{\circ} 05^{\prime} 163^{\circ} 45^{\prime}$ \\
\hline SEX & AGE (Y) & LENGTH $(\mathrm{cm})$ & WEIGHT ( $(\mathrm{kg})$ & $\begin{array}{l}\text { FLURE } \\
\text { WIDTH } \\
(\mathrm{cm})\end{array}$ & $\begin{array}{l}\text { AXILLARY } \\
\text { GRTH (cm) }\end{array}$ & BLUUBBER \\
\hline $\mathbf{M}$ & $13^{*}$ & 348 & & 82 & & 3.7 \\
\hline NBSB & NBSB & \multicolumn{2}{|c|}{ WEIGHT OF SUBSAMPLES } & & \multirow[t]{2}{*}{ HISTOLOGY } & \multirow{2}{*}{ SAMPLE } \\
\hline SAMPLES & ID No. & $A(g)$ & $\mathrm{B}(\mathrm{g})$ & & & \\
\hline Liver & MM4L137 & 169.3 & 168.1 & & yes & 26 May 92 \\
\hline Kidney & MM4K138 & 167.3 & 168.1 & & yes & \\
\hline Blubber & MM4B139 & 139.0 & 141.9 & & & 18 Jan 91 \\
\hline \multicolumn{7}{|c|}{ ADDITIONAL SAMPLES COLLECTED. } \\
\hline \multicolumn{7}{|c|}{$\begin{array}{l}\text { *Age is Based on the number of growth rings cc } \\
\text { Teeth to NSB DWM, Barrow, AK, ID \# LDL-3 } \\
\text { Histological samples to OAD, Anchorage, AK } \\
\text { Histological samples transferred from OAD to N }\end{array}$} \\
\hline
\end{tabular}

\begin{tabular}{|c|c|c|c|c|c|c|}
\hline \multicolumn{7}{|c|}{ BELUGA WHALE (Delphinapterus leucas) } \\
\hline $\begin{array}{l}\text { ANMMAL: } \\
\text { D NO. }\end{array}$ & & SAMPLING & & & LOCATION & LAT / LONG \\
\hline 692-BLKA-010 & & 11 July 90 & & & $\begin{array}{l}\text { Chukchi Sea, Point } \\
\text { Lay }\end{array}$ & $69^{\circ} 05^{\prime} 163^{\circ} 45^{\prime}$ \\
\hline SEX & AGE (y) & $\begin{array}{l}\text { TOTAL } \\
\text { LENGTH }(\mathrm{cm})\end{array}$ & $\begin{array}{l}\text { TOTAL } \\
\text { WEIGHT (kg) }\end{array}$ & $\begin{array}{l}\text { FLUKE } \\
\text { WIDTH } \\
\text { (cm) }\end{array}$ & $\begin{array}{l}\text { AXILLARY } \\
\text { GIRTH }(\mathrm{cm})\end{array}$ & $\begin{array}{l}\text { BLUBBER } \\
\text { THICKNESS }(\mathrm{cm})\end{array}$ \\
\hline $\mathbf{M}$ & $23^{*}$ & 400 & & 105 & & \\
\hline NBSB & NBSB & \multicolumn{2}{|c|}{ WEIGHT OF SUBSAMPLES } & & \multirow[t]{2}{*}{ HISTOLOGY } & \multirow{2}{*}{$\begin{array}{l}\text { SAMPLE } \\
\text { HOMOGENIZED }\end{array}$} \\
\hline SAMPLES & DNO. & $A(g)$ & $\mathrm{B}(\mathrm{g})$ & & & \\
\hline Liver & MM4L140 & 164.7 & 173.5 & & yes & 4 Sept 92 \\
\hline Kidney & MM4K141 & 176.2 & 173.5 & & yes & \\
\hline Blubber & MM4B142 & 116.3 & 116.9 & & & $23 \mathrm{Jan} 91$ \\
\hline \multicolumn{7}{|c|}{ ADDITIONAL SAMPLES COLLECTED. } \\
\hline \multicolumn{7}{|c|}{$\begin{array}{l}\text { *Age is based on the number of growth rings counted in teeth, not an absolute age. Age determined by Robert Suydam. } \\
\text { Teeth to NSB DWM, Barrow, AK, ID \# LDL-60-90 } \\
\text { Teeth, histological samples to OAD, Anchorage, AK } \\
\text { Teeth, histological samples transferred from OAD to NIST, NBSB }\end{array}$} \\
\hline
\end{tabular}




\begin{tabular}{|c|c|c|c|c|c|c|}
\hline \multicolumn{7}{|c|}{ BELUGA WHALE (Delphinapterus leucas) } \\
\hline IDNALA & & $\mathrm{SAMPLNNG}$ & & & LOCATION & LAT / LONG \\
\hline 692-BLKA-011 & & 11 July 90 & & & $\begin{array}{l}\text { Chukchi Sea, Point } \\
\text { Lay }\end{array}$ & $69^{\circ} 05^{\prime} 163^{\circ} 45^{\prime}$ \\
\hline SEX & $\mathrm{AGE}(y)$ & IENGTH $(\mathrm{cm})$ & WEIGHT $(\mathrm{kg})$ & $\begin{array}{l}\mathrm{FLUE} \\
\mathrm{WIDTH} \\
\mathrm{(cm})\end{array}$ & $\begin{array}{l}\mathrm{AXMLARY} \\
\mathrm{GLRTH}(\mathrm{cm})\end{array}$ & $\begin{array}{l}\text { BLUBBER } \\
\text { THICKNESS (cm) }\end{array}$ \\
\hline $\mathbf{M}$ & $28 *$ & 433 & & 100 & & \\
\hline \multirow{2}{*}{ SAMPLES } & \multirow{2}{*}{ NB NO } & \multicolumn{2}{|c|}{ WEIGHT OF SUBSAMPLES } & & \multirow[t]{2}{*}{ HISTOLOGY } & \multirow{2}{*}{ HOMOGENIZED } \\
\hline & & ( $\mathrm{A}(\mathrm{g})$ & & & & \\
\hline Liver & MM4L143 & 162.7 & 143.0 & & yes & 28 May 92 \\
\hline Kidney & MM4K144 & 154.35 & 165.6 & & yes & \\
\hline Blubber & MM4B145 & 95.5 & 80.6 & & & 24 Jan 91 \\
\hline \multicolumn{7}{|c|}{ ADDITIONAL SAMPLES COLLECTED } \\
\hline \multicolumn{7}{|c|}{$\begin{array}{l}\text { \#ge is based on the number of growth rings counted in teeth, not ar } \\
\text { Teeth to NSB DWM, Barrow, AK, ID \# LDL-59-90 } \\
\text { Teeth, histological samples to OAD, Anchorage, AK } \\
\text { Teeth, histological samples transferred from OAD to NIST, NBSB }\end{array}$} \\
\hline
\end{tabular}

\begin{tabular}{|c|c|c|c|c|c|c|}
\hline \multicolumn{7}{|c|}{ BELUGA WHALE (Delphinapterus leucas) } \\
\hline ANMALI & & SAMPLING & & & LOCATION & 1AT / LONG \\
\hline 692-BLKA-012 & & 11 July 90 & & & $\begin{array}{c}\text { Chukchi Sea, Point } \\
\text { Lay }\end{array}$ & $69^{\circ} 05^{\prime} 163^{\circ} 45^{\prime}$ \\
\hline $\mathrm{SEX}$ & $A G E(Y)$ & LENGTH $(\mathrm{cm})$ & WEIGHT $(\mathrm{kg})$ & FLuKE & $\begin{array}{l}\text { AXIL AR Y } \\
\text { GIRTH (cm) }\end{array}$ & THLUUBBER \\
\hline $\mathbf{F}$ & $55^{*}$ & 375 & & 84 & & \\
\hline \multirow{2}{*}{ SAMPLES } & \multirow{2}{*}{ NBSB } & \multicolumn{2}{|c|}{ WEIGHT OF SUBS AMPLES } & & \multirow[t]{2}{*}{ HISTOLOGY } & \multirow{2}{*}{ HOMOGENIZED } \\
\hline & & $A(g)$ & $\mathrm{B}(\mathrm{g})$ & & & \\
\hline Liver & MM4L146 & 185.2 & 169.75 & & yes & 24 Oct 90 \\
\hline Kidney & MM4K147 & 156.6 & 172.25 & & yes & \\
\hline Blubber & MM4B148 & 142.55 & 133.45 & & & 2 Oct 90 \\
\hline \multicolumn{7}{|c|}{ ADDITIONAL SAMPLES COLLECTED } \\
\hline \multicolumn{7}{|c|}{$\begin{array}{l}\text { *Age is based on the number of growth rings counted in teeth, not } \\
\text { Teeth, reporductive organs to NSB DWM, Barrow, AK, ID \# LDL } \\
\text { Teeth, histological samples to OAD, Anchorage, AK } \\
\text { Teeth, histological samples transferred from OAD to NIST, NBSB }\end{array}$} \\
\hline
\end{tabular}




\begin{tabular}{|c|c|c|c|c|c|c|}
\hline \multicolumn{7}{|c|}{ BELUGA WHALE (Delphinapterus leucas) } \\
\hline ANMMAL & & SAMPLING & & & LOCATION & LAT / LONG \\
\hline 692-BLKA-013 & & 11 July 90 & & & $\begin{array}{l}\text { Chukchi Sea, Point } \\
\text { Lay }\end{array}$ & $69^{\circ} 05^{\prime} 163^{\circ} 45^{\prime}$ \\
\hline SEX & AGE ( & $\begin{array}{l}\text { TOTAL } \\
\text { UENGTH }(\mathrm{cm}) \text { ? }\end{array}$ & TEIGHT ( $\mathrm{gg}$ ) & $\begin{array}{l}\text { FLURE } \\
\text { WIDTH } \\
\text { (cm) }\end{array}$ & $\begin{array}{l}\text { AXIILAARY } \\
\text { GIRTH (cm) }\end{array}$ & $\begin{array}{l}\text { BLUBBER } \\
\text { THICKNESS (cm) }\end{array}$ \\
\hline M & $26^{*}$ & 434 & & 98 & & 8.4 \\
\hline NBSB & NBSB & \multicolumn{2}{|c|}{ WEIGHT OF SUBSAMPLES } & & \multirow[t]{2}{*}{ HISTOLOGY } & \multirow{2}{*}{$\begin{array}{l}\text { SAMPLE } \\
\text { HOMOGENIZED }\end{array}$} \\
\hline SAMPLES & WONO & $A(g)$ & $\mathrm{B}(\mathrm{g})$ & & & \\
\hline Liver & MM4L149 & 161.0 & 168.1 & & yes & 2 Jun 92 \\
\hline Kidney & MM4K150 & 179.8 & 160.95 & & yes & \\
\hline Blubber & MM4B151 & 147.1 & 148.25 & & & $28 \operatorname{Jan} 91$ \\
\hline \multicolumn{7}{|c|}{ ADDITIONAL SAMPLES COLLECTED; } \\
\hline \multicolumn{7}{|c|}{$\begin{array}{l}\text { *Age is based on the number of growth rings counted in teeth, not an absolute age. Age determined by Robert Suydam. } \\
\text { Teeth, skin to NSB DWM, Barrow, AK, ID \# LDL-16-90 } \\
\text { Teeth, histological samples to OAD, Anchorage, AK } \\
\text { Teeth, histological samples transferred from OAD to NIST, NBSB }\end{array}$} \\
\hline
\end{tabular}

\begin{tabular}{|c|c|c|c|c|c|c|}
\hline \multicolumn{7}{|c|}{ BELUGA WHALE (Delphinapterus leucas) } \\
\hline $\begin{array}{l}\text { ANMALI } \\
\text { ID NO. }\end{array}$ & & $\begin{array}{l}\text { SAMPLING } \\
\text { DATE }\end{array}$ & & & LOCATION & LAT / LONG \\
\hline 692-BLKA-014 & & 11 July 90 & & & $\begin{array}{c}\text { Chukchi Sea, Point } \\
\text { Lay }\end{array}$ & $69^{\circ} 05^{\prime} 163^{\circ} 45^{\prime}$ \\
\hline SEX & AGE $(y)$ & LENGTH (cm) & WEIGHT ( $\mathrm{kg}$ ) & $\begin{array}{l}\text { FLUKR } \\
\text { WIDTH } \\
(\mathrm{cm})\end{array}$ & $\begin{array}{l}\text { AXILLARY } \\
\text { GIRTH (crn) }\end{array}$ & $\begin{array}{l}\text { BLUBBER } \\
\text { THICKNESS (cm) }\end{array}$ \\
\hline $\mathbf{F}$ & $23 *$ & 351 & & 82 & & \\
\hline \multirow{2}{*}{ NAMPB } & \multirow{2}{*}{$\begin{array}{l}\text { NBSB } \\
\text { ID NO. }\end{array}$} & \multicolumn{2}{|c|}{ WEIGHT OF SUBS AMPLES } & & \multirow[t]{2}{*}{ HISTOLOGY } & \multirow{2}{*}{$\begin{array}{l}\text { SAMPLE } \\
\text { HOMOGENIZED }\end{array}$} \\
\hline & & $A(g)$ & B (g) & & & \\
\hline Liver & MM4L152 & $16 \hat{i} .0$ & 172.85 & & yes & 9 Jun 92 \\
\hline Kidney & MM4K153 & 158.1 & 156.2 & & yes & \\
\hline Blubber & MM4B15i & 146.7 & 149.9 & & & $1 \mathrm{Feb} 91$ \\
\hline & & & & & & \\
\hline \multicolumn{7}{|c|}{ ADDTIONAL SAMPLES COLLECTED: } \\
\hline \multicolumn{7}{|c|}{$\begin{array}{l}\text { *Age is based on the number of growth rings counted in teeth, not an absolute age. Age determined by Robert Suydam. } \\
\text { Teeth, reporductive organs to NSB DWM, Barrow, AK, ID \# LDL-9-90 } \\
\text { Teeth, histological samples to OAD, Anchorage, AK } \\
\text { Teeth, histological samples transferred from OAD to NIST, NBSB }\end{array}$} \\
\hline
\end{tabular}




\begin{tabular}{|c|c|c|c|c|c|c|}
\hline \multicolumn{7}{|c|}{ BELUGA WHALE (Delphinapterus leucas) } \\
\hline $\begin{array}{l}\text { ANIMAI } \\
\text { ID NO. }\end{array}$ & & SAMPLING & & & LOCATION & LAT / LONG \\
\hline 692-BLKA-015 & & 6 Oct 92 & & & Cook Inlet & $60^{\circ} 35.1^{\prime} 151^{\circ} 19.0^{\prime}$ \\
\hline SEX & $\mathrm{AGE}\left(\mathrm{O}^{\prime}\right)$ & $\begin{array}{l}\text { TOTAL } \\
\text { LENGTH (cm) }\end{array}$ & $\begin{array}{l}\text { TOTAI , } \\
\text { WEIGHT }(\mathrm{kg}) \text {. }\end{array}$ & $\begin{array}{l}\text { FLUKE } \\
\text { WDTH } \\
\text { (cm) }\end{array}$ & $\begin{array}{l}\text { AXIl LARY } \\
\text { GIRTH (cm) }\end{array}$ & THICKNESS (cm) \\
\hline $\mathbf{M}$ & Mature & 373.3 & & 78.7 & & 15.5 \\
\hline NBSB & NBSB & \multicolumn{2}{|c|}{ WEIGHT OF SUBSAMPLES } & & HISTOLOGY & SAMPLE \\
\hline SAMPLES & DNO. & $A(g)$ & $\mathrm{B}(\mathrm{g})$ & & & HOMOGENIZED \\
\hline Liver & MM6L216 & 170.9 & 175.4 & & & 19 Jan 93 \\
\hline Kidney & MM6K217 & 157.3 & 147.6 & & & \\
\hline Blubber & MM6B218 & 138.3 & 151.7 & & & \\
\hline \multicolumn{7}{|c|}{ ADDITIONAI SAMPLES COLLECTED: } \\
\hline \multicolumn{7}{|c|}{$\begin{array}{l}\text { NMFS Western Alaska Field Office, ID \# Kenai Beluga } 3 \\
\text { Skin, teeth to NMML, Seattle, WA } \\
\text { Liver to U of AK Museum, Alaska Frozen Tissue Collection, Fairbanks, AK } \\
\text { Skin to ADF\&G, Fairbanks, AK }\end{array}$} \\
\hline
\end{tabular}

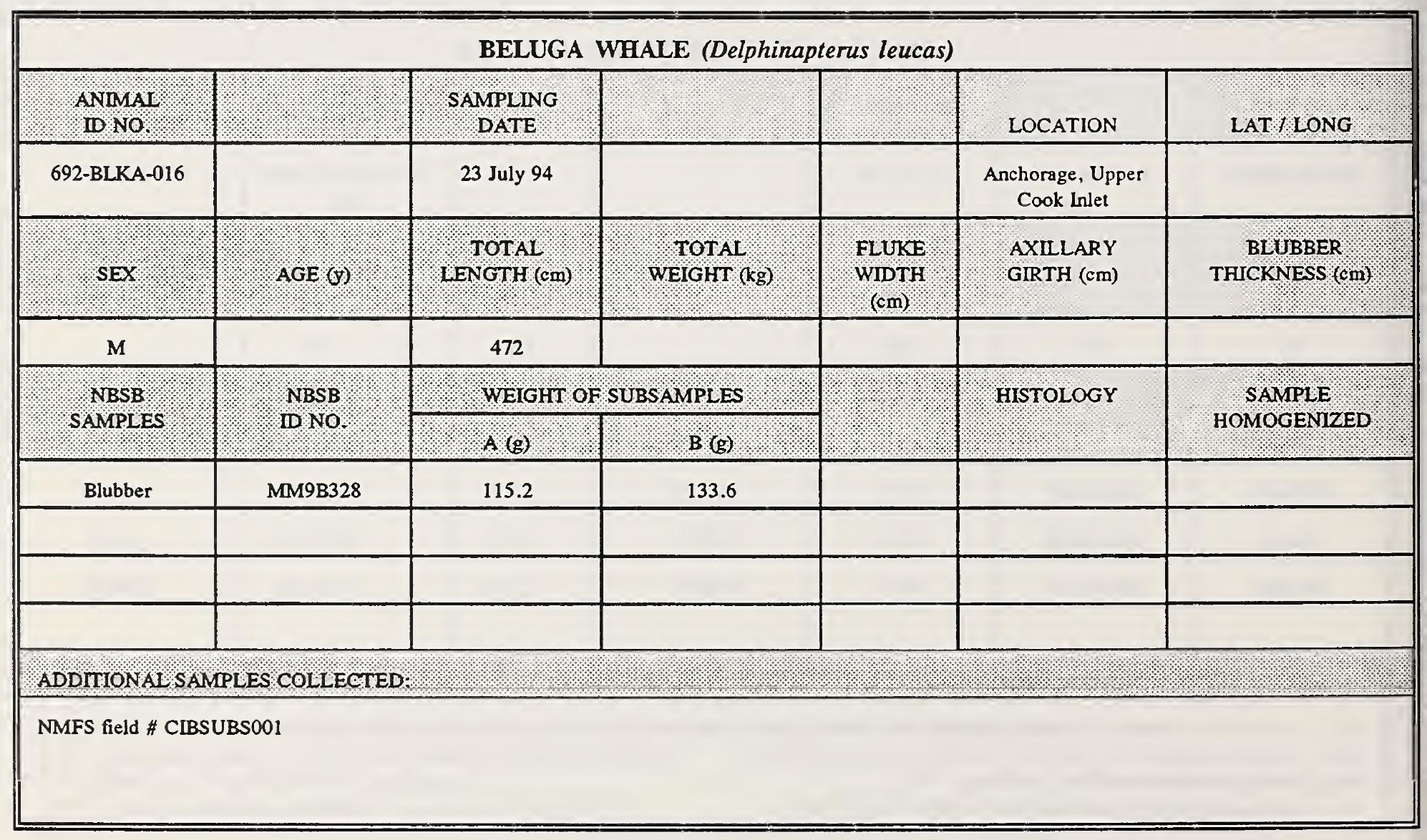




\begin{tabular}{|c|c|c|c|c|c|c|}
\hline \multicolumn{7}{|c|}{ BELUGA WHALE (Delphinapterus leucas) } \\
\hline ANMALI & & $\begin{array}{l}\text { SAMPLING } \\
\text { DATE }\end{array}$ & & & LOCATION & LAT / LONG \\
\hline 692-BLKA-017 & & 22 July 94 & & & $\begin{array}{c}\text { Anchorage, Upper } \\
\text { Cook Inlet }\end{array}$ & \\
\hline SEX & AGE (y) & $\begin{array}{l}\text { TOTAI: } \\
\text { LENGTH (cm) }\end{array}$ & $\begin{array}{l}\text { TOTAI } \\
\text { WEIGHT }(\mathrm{kg})\end{array}$ & $\begin{array}{l}\text { FLURE } \\
\text { WIDTH } \\
\text { (cm) }\end{array}$ & $\begin{array}{l}\text { AXILLARY } \\
\text { GIRTH (cm) }\end{array}$ & THICKNESS $(\mathrm{cm})$ \\
\hline $\mathbf{F}$ & & 305 & & & & \\
\hline NBSB & \multirow{2}{*}{ NBSB } & \multicolumn{2}{|c|}{ WEIGHT OF SUBS AMPLES } & & \multirow[t]{2}{*}{ HISTOLOGY } & \multirow{2}{*}{ HOMOGENIZED } \\
\hline SAvir & & $A(g)$ & & & & \\
\hline Blubber & MM9B329 & 117.5 & 126.4 & & & \\
\hline & & & & & & \\
\hline & & & & & & \\
\hline & & & & & & \\
\hline \multicolumn{7}{|c|}{ ADDITIONAL SAMPLES COLLECTED: } \\
\hline \multicolumn{7}{|c|}{ NMFS field \# CLBSUBSO02 } \\
\hline
\end{tabular}

\begin{tabular}{|c|c|c|c|c|c|c|}
\hline \multicolumn{7}{|c|}{ BELUGA WHALE (Delphinapterus leucas) } \\
\hline $\begin{array}{l}\text { ANIMAI } \\
\text { D NO. }\end{array}$ & & $\begin{array}{l}\text { SAMPLING } \\
\text { DATE }\end{array}$ & & & LOCATION & LAT / LONG \\
\hline 692-BLKA-018 & & 23 July 94 & & & $\begin{array}{l}\text { Anchorage, Upper } \\
\text { Cook Inlet }\end{array}$ & \\
\hline SEX & AGE $(g)$ & $\begin{array}{l}\text { TOTAI : } \\
\text { LENGTH }(\mathrm{cm}) \text {. }\end{array}$ & $\begin{array}{l}\text { TOTAL } \\
\text { WEIGHT (kg) }\end{array}$ & $\begin{array}{l}\text { FLUKE } \\
\text { WIDTH } \\
(\mathrm{cm})\end{array}$ & $\begin{array}{l}\text { AXILLARY } \\
\text { GIRTH (cm) }\end{array}$ & THICKNESS (cm) \\
\hline $\mathbf{M}$ & & 305 & & & & \\
\hline \multirow{2}{*}{ SAMPLES } & \multirow{2}{*}{ NBSB } & \multicolumn{2}{|c|}{ WEIGHT OF SUBS AMPLES } & & \multirow[t]{2}{*}{ HISTOLOGY } & \multirow{2}{*}{$\begin{array}{l}\text { SAMPLE } \\
\text { HOMOGENIZED }\end{array}$} \\
\hline & & $A(g)$ & B (g) & & & \\
\hline Blubber & MM9B330 & 94.1 & 73.0 & & & \\
\hline & & & & & & \\
\hline & & & & & & \\
\hline & & & & & & \\
\hline \multicolumn{7}{|c|}{ ADDIMONAL SAMPLES COLLECTED. } \\
\hline \multicolumn{7}{|c|}{ NMFS field \# CIBSUBS003 } \\
\hline
\end{tabular}




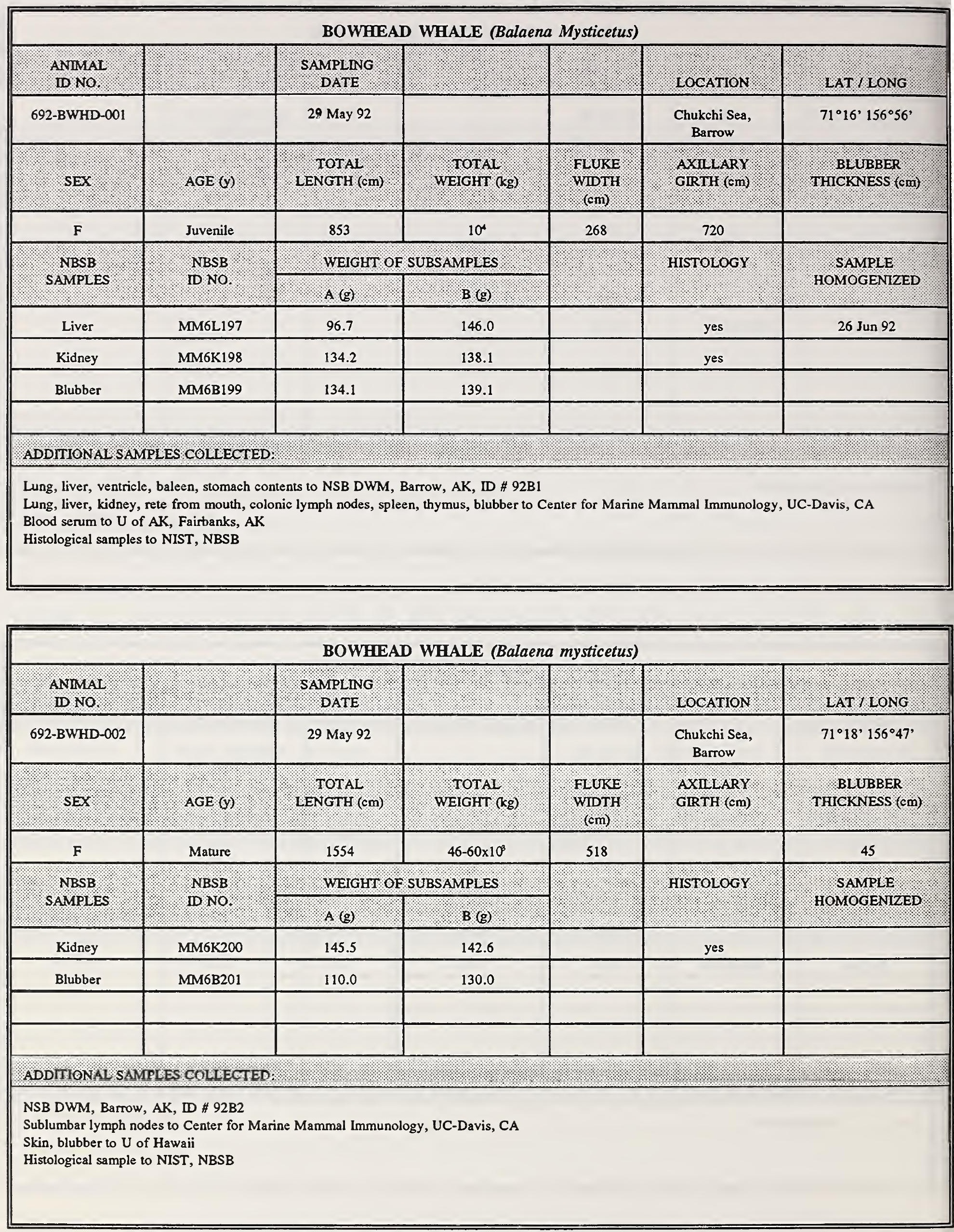




\begin{tabular}{|c|c|c|c|c|c|c|}
\hline \multicolumn{7}{|c|}{ BOWHEAD WHALE (Balaena mysticetus) } \\
\hline ANIM NOL, & & $\begin{array}{l}\text { SAMPLING } \\
\text { DATE }\end{array}$ & & . & LOCATION & LAT / LONG \\
\hline 692-BWHD-003 & & 12 Sept 92 & & & $\begin{array}{l}\text { Chukchi Sea, } \\
\text { Barrow }\end{array}$ & $71^{\circ} 25^{\prime} 155^{\circ} 49^{\prime}$ \\
\hline $\operatorname{sex}$. & AGE (y) & $\begin{array}{l}\text { TOTAL } \\
\text { LENGTH (cm) }\end{array}$ & $\begin{array}{l}\text { TOTAL } \\
\text { WEIGHT (kg) }\end{array}$ & $\begin{array}{l}\text { FLURE } \\
\text { WIDTH } \\
\text { (cm) }\end{array}$ & $\begin{array}{l}\text { AXILLARY } \\
\text { GIRTH }(\mathrm{cm})\end{array}$ & $\begin{array}{l}\text { BLUBBER } \\
\text { THICKNESS (cm) }\end{array}$ \\
\hline $\mathbf{F}$ & Mature & 1460.5 & & & 946 & 23 \\
\hline NBSB & NBSB & \multicolumn{2}{|c|}{ WEIGHT OF SUBSAMPLES } & & \multirow[t]{2}{*}{ HistoLOGY } & \multirow{2}{*}{ HOMOGENIZED } \\
\hline SAMPLES & ID NO: & $A(g)$ & B (g) & & & \\
\hline Liver & MM6L202 & 170.9 & 176.9 & & yes & \\
\hline Blubber & MM6B203 & 180.0 & 157.6 & & & \\
\hline & & & & & & \\
\hline \multicolumn{7}{|c|}{ ADDITIONAL SAMPLES COLLECTED. } \\
\hline \multicolumn{7}{|c|}{$\begin{array}{l}\text { Ovaries, stomach contents, skin with blubber to NSB DWM, Barrow, AK, ID \# 92B10 } \\
\text { Blubber, liver, kidney to NMFS, Seattle, WA } \\
\text { Blubber, liver, kidney to U of Ulm, Ulm, Germany } \\
\text { Blood serum to U of AK, Fairbanks, AK } \\
\text { Skin, blubber to U of Hawaii } \\
\text { Histological sample to NIST, NBSB }\end{array}$} \\
\hline
\end{tabular}

\begin{tabular}{|c|c|c|c|c|c|c|}
\hline \multicolumn{7}{|c|}{ BOWHEAD WHALE (Balaena mysticetus) } \\
\hline $\begin{array}{l}\text { ANIMAL } \\
\text { ID NO. }\end{array}$ & & SAMPLING & & & LOCATION & LAT / LONG \\
\hline 692-BWHD-004 & & 17 Sept 92 & & & $\begin{array}{l}\text { Chukchi Sea, } \\
\text { Barrow }\end{array}$ & $71^{\circ} 25^{\prime} 155^{\circ} 49^{\prime}$ \\
\hline sEX & AGE $(y)$ & LENGTH $(\mathrm{cm})$ & $\begin{array}{l}\text { TOTAL } \\
\text { WEIGHT (kg) }\end{array}$ & $\begin{array}{l}\text { FLURE } \\
\text { WIDTH } \\
(\mathrm{cm})\end{array}$ & $\begin{array}{l}\text { AXILLARY } \\
\text { GIRTH (cm) }\end{array}$ & $\begin{array}{l}\text { BLUBBER } \\
\text { THICKNESS }(\mathrm{cm})\end{array}$ \\
\hline $\mathbf{M}$ & Mature & 1504 & & & 960 & 26 \\
\hline \multirow{2}{*}{ SAMPLES } & \multirow{2}{*}{ NBSB } & \multicolumn{2}{|c|}{ WEIGHT OF SUBSAMPLES } & & \multirow[t]{2}{*}{ HISTOLOGY } & \multirow{2}{*}{$\begin{array}{l}\text { SAMPLE } \\
\text { HOMOGENIZED }\end{array}$} \\
\hline & & $A(g)$ & $\mathrm{B}(\mathrm{g})$ & & & \\
\hline Liver & MM6L204 & 180.0 & 175.3 & & yes & \\
\hline Blubber & MM6B205 & 144.2 & 123.6 & & & \\
\hline & & & & & & \\
\hline \multirow{2}{*}{\multicolumn{7}{|c|}{ ADDIIIONAL SAMPLES COLLECTED: }} \\
\hline & & & & & & \\
\hline \multicolumn{7}{|c|}{$\begin{array}{l}\text { Spleen, heart section, testes section, skin lesions } \\
\text { AK, ID \# 92B11 } \\
\text { Blubber with skin, liver to NMFS, Seattle, WA } \\
\text { Blubber, liver to U of Ulm, Ulm, Germany } \\
\text { Blood serum to U of AK, Fairbanks, AK } \\
\text { Skin, blubber to U of Hawaii } \\
\text { Histological sample to NIST, NBSB }\end{array}$} \\
\hline
\end{tabular}




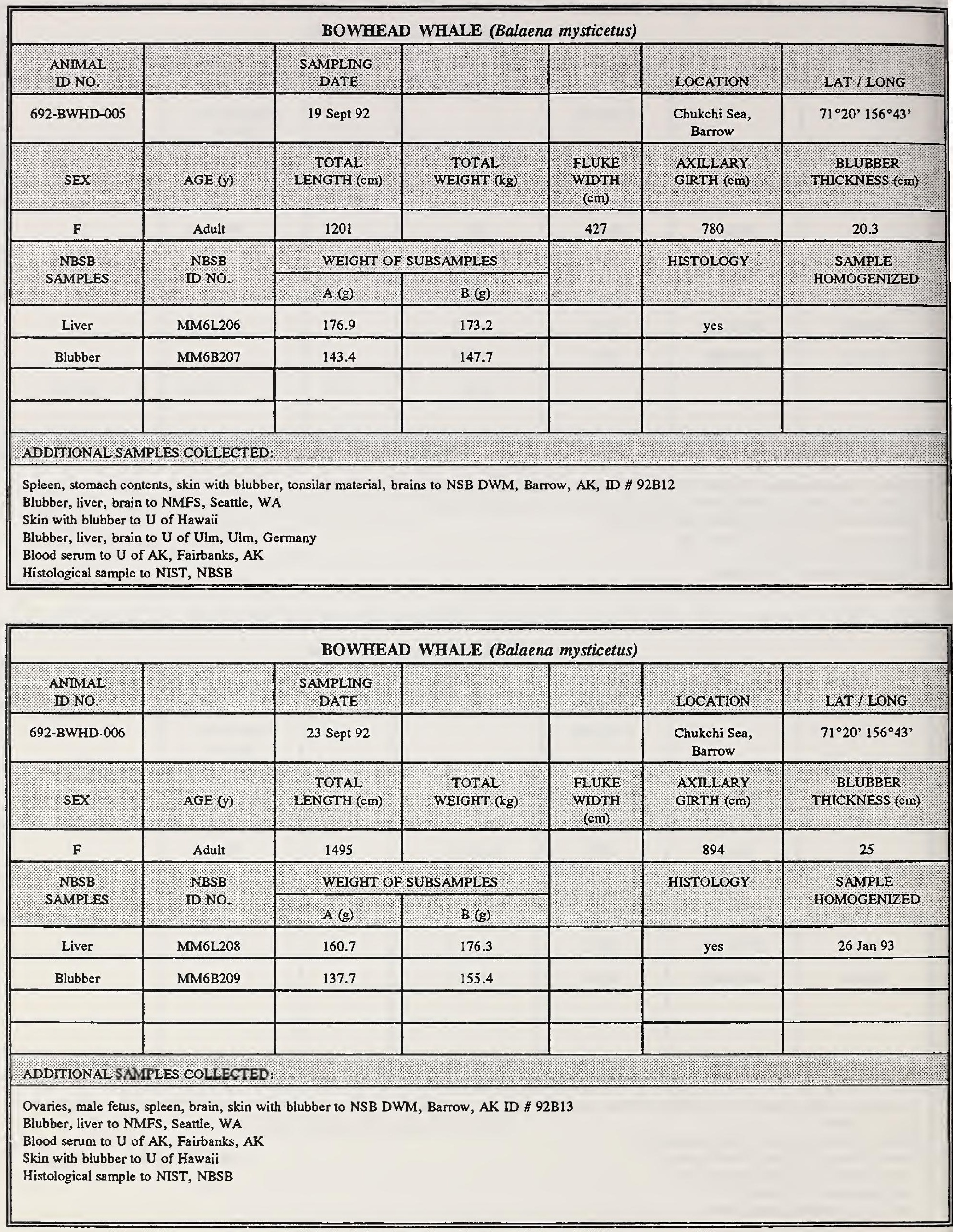




\begin{tabular}{|c|c|c|c|c|c|c|}
\hline \multicolumn{7}{|c|}{ BOWHEAD WHALE (Balaena mysticetus) } \\
\hline ANMMAl: & & SAMPLING & & & LOCATION & LAT / LONG \\
\hline 692-BWHD-007 & & 24 Sept 92 & & & $\begin{array}{c}\text { Chukchi Sea, } \\
\text { Barrow }\end{array}$ & $71^{\circ} 20^{\prime} 156^{\circ} 43^{\prime}$ \\
\hline SEX & AGE (y) & IENGTH $(\mathrm{cm})$ T & 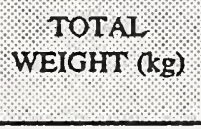 & $\begin{array}{l}\text { FLURE } \\
\text { WIDTH } \\
\text { (cm) }\end{array}$ & $\begin{array}{l}\text { AXILLARY } \\
\text { GIRTH (cm) }\end{array}$ & $\begin{array}{l}\text { BLUBBER } \\
\text { THICKNESS (cm) }\end{array}$ \\
\hline $\mathbf{M}$ & Adult & 1450 & & & 898 & 20.5 \\
\hline \multirow{2}{*}{$\begin{array}{l}\text { NBSB } \\
\text { SAMPLES }\end{array}$} & \multirow{2}{*}{ ID No. } & \multicolumn{2}{|c|}{ WEIGHT OF SUBSAMPLES } & & \multirow[t]{2}{*}{ HISTOLOGY } & \multirow{2}{*}{, SOMOGENIZED } \\
\hline & & $A(g)$ & $\mathrm{B}(\mathrm{g})$ & & & \\
\hline Liver & MM6L210 & 160.9 & 157.4 & & yes & $27 \mathrm{Jan} 93$ \\
\hline Blubber & MM6B211 & 139.8 & 175.8 & & & 26 May 93 \\
\hline & & & & & & \\
\hline & & & & & & \\
\hline \multicolumn{7}{|c|}{ ADDMIONAL SAMPLES COLLECTED: } \\
\hline \multicolumn{7}{|c|}{$\begin{array}{l}\text { Testes section, brain, skin with blubber to NSB DWM, Barrow, AK, ID \# 92B14 } \\
\text { Blubber, liver to NMFS, Seattle, WA } \\
\text { Heart, kidney, liver, muscle to U of AK Museum, Fairbanks, AK } \\
\text { Blood serum to U of AK, Fairbanks, AK } \\
\text { Skin with blubber to U of Hawaii } \\
\text { Histological sample to NIST, NBSB }\end{array}$} \\
\hline
\end{tabular}

\begin{tabular}{|c|c|c|c|c|c|c|}
\hline \multicolumn{7}{|c|}{ BOWHEAD WHALE (Balaena mysticetus) } \\
\hline $\begin{array}{l}\text { ANDAL } \\
\text { ID NO. }\end{array}$ & & $\begin{array}{l}\text { SAMPLING } \\
\text { DATE }\end{array}$ & & & LOCATION & LAT / LONG \\
\hline 692-BWHD-008 & & 26 Sept 92 & & & $\begin{array}{c}\text { Chukchi Sea, } \\
\text { Barrow }\end{array}$ & $71^{\circ} 20^{\prime} 156^{\circ} 43^{\prime}$ \\
\hline SEX & AGE $(y)$ & $\begin{array}{l}\text {. TOTAL } \\
\text { LENGTH (com) }\end{array}$ & $\begin{array}{l}\text { TOTAL } \\
\text { WEIGHT (kg) }\end{array}$ & $\begin{array}{l}\text { FLURE } \\
\text { WIDTH } \\
(\mathrm{cm})\end{array}$ & $\begin{array}{l}\text { AXILLARY } \\
\text { GIRTH (cm) }\end{array}$ & THICKNESS (cm) \\
\hline $\mathbf{M}$ & & 1170 & & & 736 & 25 \\
\hline \multirow{2}{*}{ SAMPLES } & \multirow{2}{*}{ NBSB } & \multicolumn{2}{|c|}{ WEIGHT OF SUBSAMPLES } & & \multirow[t]{2}{*}{ HISTOLOGY } & \multirow{2}{*}{ HOMOGENIZED } \\
\hline & & A (g) & $B(g)$ & & & \\
\hline Liver & MM6L212 & 164.7 & 175.9 & & yes & \\
\hline Blubber & MM6B213 & 147.3 & 132.3 & & & \\
\hline & & & & & & \\
\hline & & & & & & \\
\hline \multicolumn{7}{|c|}{ ADDITIONAL SAMPLES COLLECTED. } \\
\hline \multicolumn{7}{|c|}{$\begin{array}{l}\text { Testes section, } 36 \text { skin/blubber samples, spleen, bulbourethral gland, urine, penis \& attachments, urinary bladder to NSB DWM, Barrow, AK, ID \#92B15 } \\
\text { Blubber, liver to NMFS, Seatte, WA } \\
\text { Liver, muscle to U of AK Museum, Fairbanks, AK } \\
\text { Blood serum to U of AK, Fairbanks, AK } \\
\text { Skin, blubber to U of Hawaii } \\
\text { Histological samples to NIST, NBSB }\end{array}$} \\
\hline
\end{tabular}




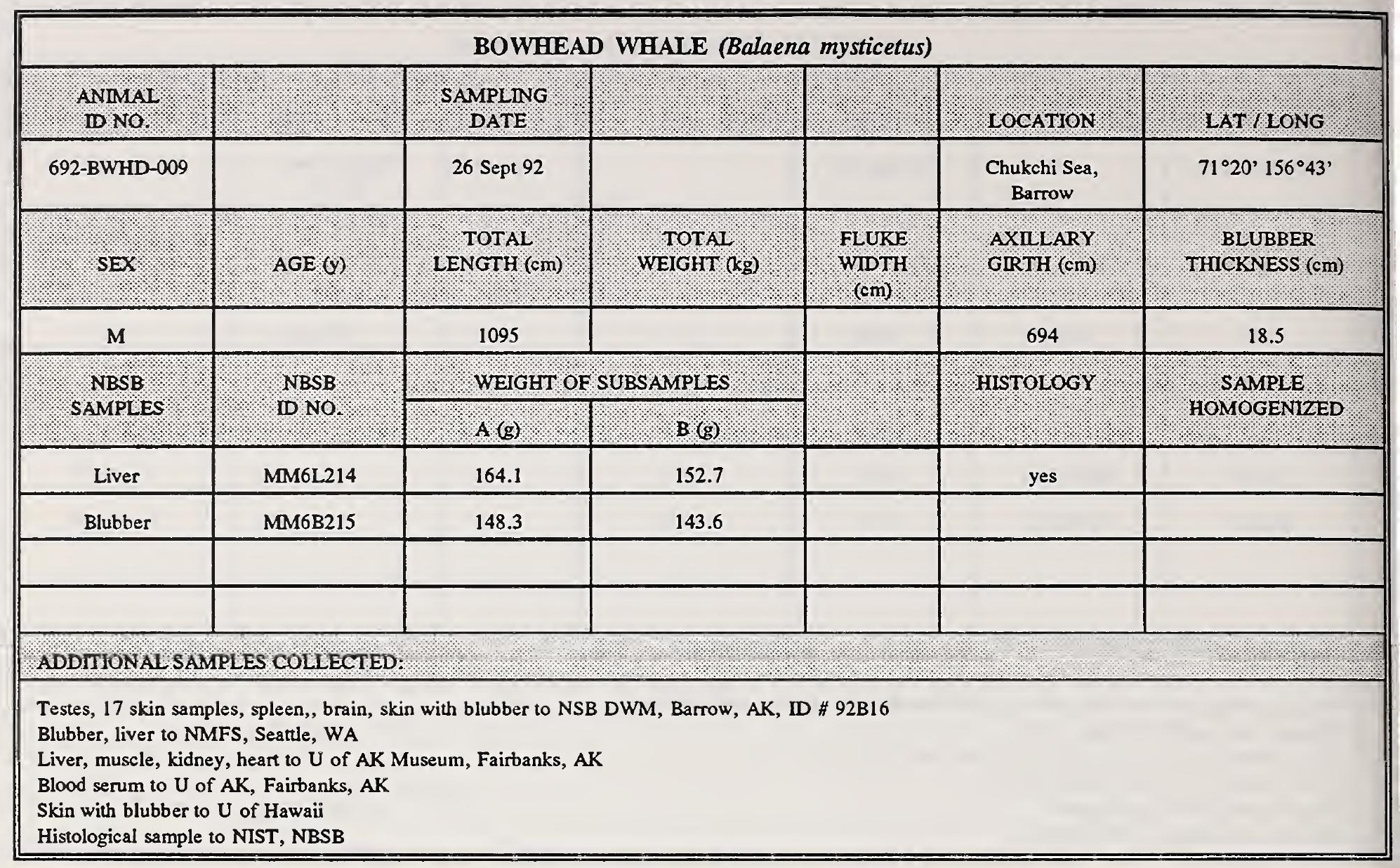

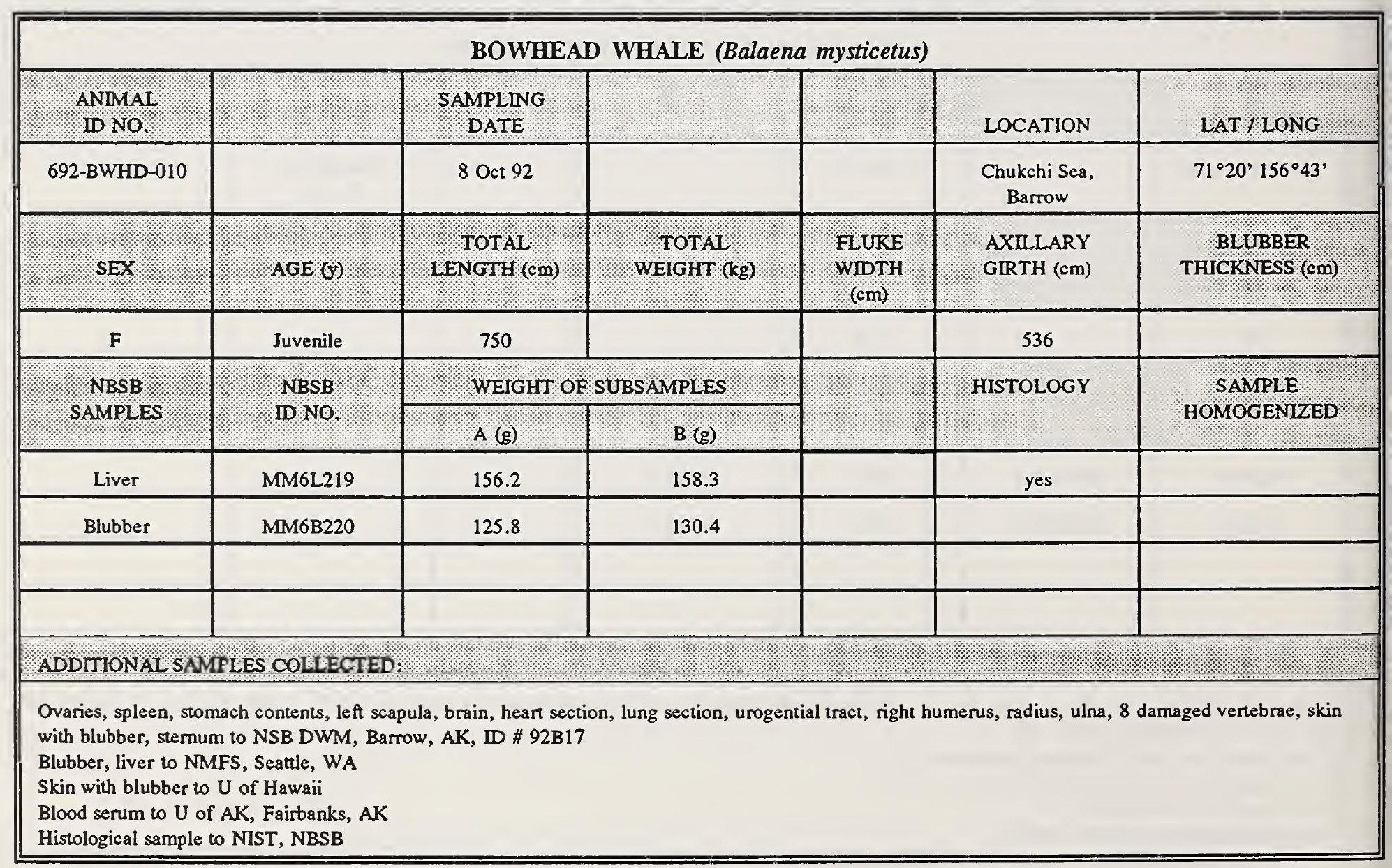




\begin{tabular}{|c|c|c|c|c|c|c|}
\hline \multicolumn{7}{|c|}{ BOWHEAD WHALE (Balaena mysticetus) } \\
\hline ANMAAl: & & $\begin{array}{l}\text { SAMPLING } \\
\text { DATE }\end{array}$ & & & LOCATION & LAT / LONG \\
\hline 692-BWHD-011 & & 9 Oct 92 & & & $\begin{array}{c}\text { Chukchi Sea, } \\
\text { Barrow }\end{array}$ & $71^{\circ} 20^{\prime} 156^{\circ} 43^{\prime}$ \\
\hline SEX & AGE ( & $\begin{array}{l}\text { TOTAL } \\
\text { LENGTH (cm) }\end{array}$ & WEIGHT (og) & $\begin{array}{l}\text { FLURE } \\
\text { WIDTH } \\
(\mathrm{cm})\end{array}$ & $\begin{array}{l}\text { AXILLARY } \\
\text { GRTH (cm) }\end{array}$ & $\begin{array}{l}\text { BLUBBER } \\
\text { THICKNESS (cm) }\end{array}$ \\
\hline $\mathbf{M}$ & & Juvenile & 876 & & & 608 \\
\hline \multirow{2}{*}{$\begin{array}{l}\text { NABSB } \\
\text { SAMPLES }\end{array}$} & \multirow{2}{*}{ NBSB } & \multicolumn{2}{|c|}{ ISWEIGHT OF SUBSAMPLES } & & \multirow[t]{2}{*}{ HISTOLOGY } & \multirow{2}{*}{ SAMPLE } \\
\hline & & $A(g)$ & B (g) & & & \\
\hline Liver & MM6L221 & 149.4 & 146.2 & & yes & \\
\hline Blubber & MM6B222 & 135.6 & 119.2 & & & \\
\hline & & & & & & \\
\hline & & & & & & \\
\hline \multicolumn{7}{|c|}{ ADDITIONAL SAMPLES COLLECTED: } \\
\hline \multicolumn{7}{|c|}{$\begin{array}{l}\text { Spleen, brain, urogential tract, stomach contents, skin with blubt } \\
\text { Blubber, liver to NMFS, Seattle, WA } \\
\text { Skin with blubber to U of Hawaii } \\
\text { Blood serum to U of AK, Fairbanks, AK } \\
\text { Histological samples to NIST, NBSB }\end{array}$} \\
\hline
\end{tabular}

\begin{tabular}{|c|c|c|c|c|c|c|}
\hline \multicolumn{7}{|c|}{ BOWHEAD WHALE (Balaena mysticetus) } \\
\hline $\begin{array}{l}\text { ANMMAL } \\
\text { D NO }\end{array}$ & & $\begin{array}{l}\text { SAMPLING } \\
\text { DATE }\end{array}$ & & & LOCATION & LAT / LONG \\
\hline 692-BWHD-012 & & 30 April 93 & & & $\begin{array}{c}\text { Chukchi Sea, } \\
\text { Barrow }\end{array}$ & $71^{\circ} 20^{\prime} 156^{\circ} 43^{\prime}$ \\
\hline SEX & $\mathrm{AGE}(y)$ & $\begin{array}{l}\text { TOTAL: } \\
\text { LENGTH. (com) }\end{array}$ & WEIGHT ( T & $\begin{array}{l}\text { FLURE } \\
\text { (1DTH } \\
(\mathrm{cm})\end{array}$ & $\begin{array}{l}\text { AXILLARY } \\
\text { GRTH (cm) }\end{array}$ & THICKNESS $(\mathrm{cm})$ \\
\hline F & & 899 & & 299 & 576 & 20 \\
\hline \multirow{2}{*}{ SAMPLES } & \multirow{2}{*}{$\begin{array}{l}\text { NBSB } \\
\text { D NO. }\end{array}$} & \multicolumn{2}{|c|}{ WEIGHT OF SUBSAMPLES } & & \multirow[t]{2}{*}{ HISTOLOGY } & \multirow{2}{*}{ SAMPLE } \\
\hline & & $A(g)$ & $\mathrm{B}(\mathrm{g})$ & & & \\
\hline Liver & MM7L235 & 174.5 & 180.3 & & yes & \\
\hline Blubber & MM7B236 & 142.5 & 143.4 & & & \\
\hline & & & & & & \\
\hline \multicolumn{7}{|c|}{ ADDITIONAL SAMPLES COLLECTED. } \\
\hline \multicolumn{7}{|c|}{$\begin{array}{l}\text { NSB DWM, Barrow, AK, ID \# 93B7 } \\
\text { Blubber, liver to NMFS, Seattle, WA } \\
\text { Skin with blubber to U of Hawaii } \\
\text { Blood serum to U of AK, Fairbanks, AK } \\
\text { Histological samples to NIST, NBSB }\end{array}$} \\
\hline
\end{tabular}




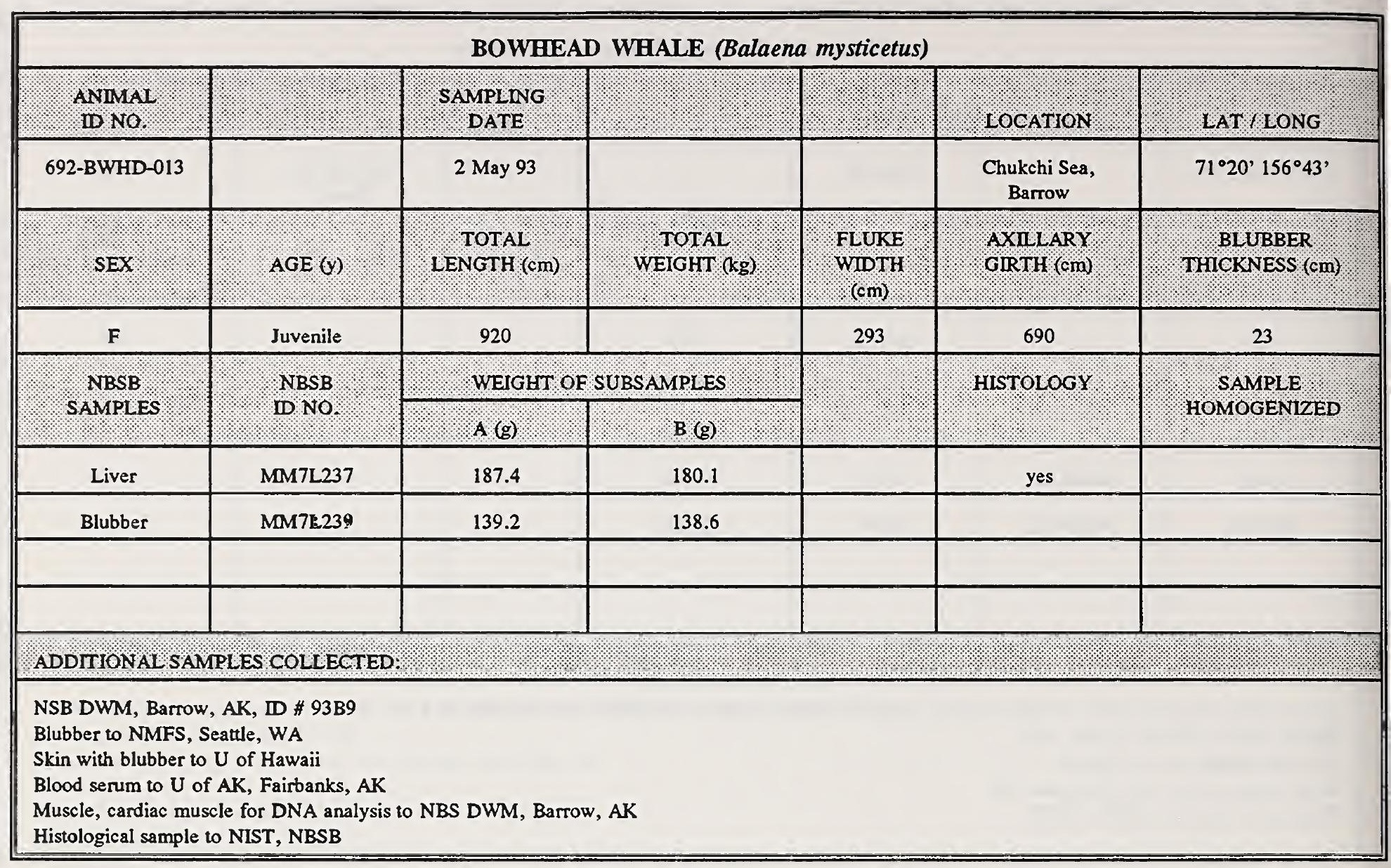

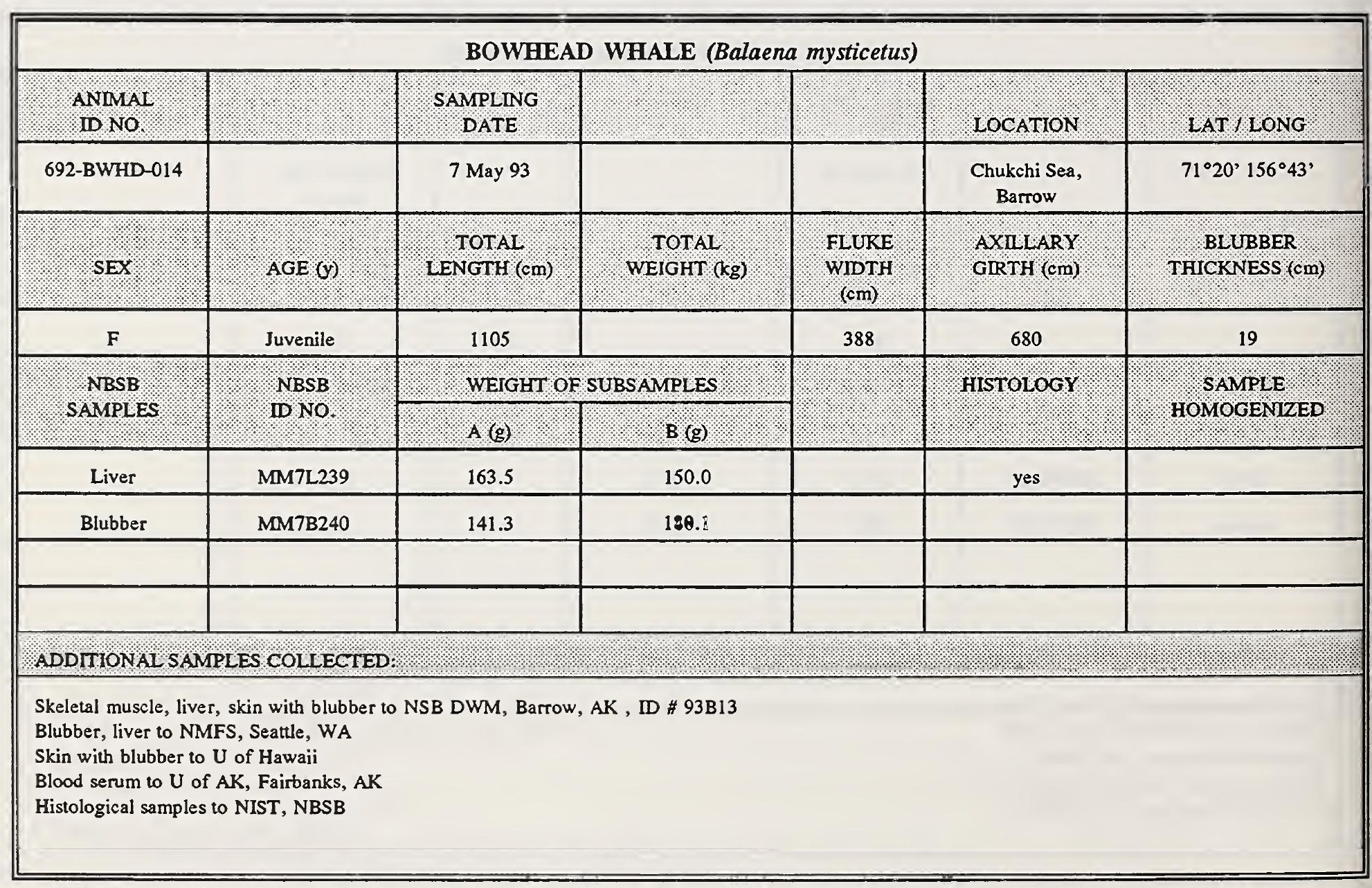




\begin{tabular}{|c|c|c|c|c|c|c|}
\hline \multicolumn{7}{|c|}{ BOWHEAD WHALE (Balaena mysticetus) } \\
\hline INIMAL & & SAMPLING & & & LOCATION & LAT / LONG \\
\hline 692-BWHD-015 & & 7 May 93 & & & $\begin{array}{c}\text { Chukchi Sea, } \\
\text { Barrow }\end{array}$ & $71^{\circ} 20^{\prime} 156^{\circ} 43^{\prime}$ \\
\hline SEX & AGE $(y)$ & I. TONGTH (cm) & $\begin{array}{l}\text { TOTAL : } \\
\text { WEIGHT (kg) }\end{array}$ & $\begin{array}{l}\text { FLUKE } \\
\text { WIDTH } \\
(\mathrm{cm})\end{array}$ & $\begin{array}{l}\text { AXILLARY } \\
\text { GIRTH (cm) }\end{array}$ & BHCKNBES (cm) \\
\hline F & Juvenile & 1045 & & 352 & 674 & 15 \\
\hline , NBSB & NBSB & \multicolumn{2}{|c|}{ WEIGHT OF SUBS AMPLES } & & \multirow[t]{2}{*}{ HistologY Y } & \multirow{2}{*}{ SAMOGENIZED } \\
\hline SAMPLES & ID NO. & $A(g)$ & B (g) & & & \\
\hline Liver & MM7L241 & 151.5 & 166.1 & & yes & \\
\hline Blubber & MM7B242 & 147.0 & 159.7 & & & \\
\hline & & & & & & \\
\hline & & & & & & \\
\hline \multicolumn{7}{|c|}{ ADDITIONAL SAMPLES COLLECTED: } \\
\hline \multicolumn{7}{|c|}{$\begin{array}{l}\text { Myocardium, skeletal muscle, skin with bl } \\
\text { Blubber, liver to NMFS, Seattle, WA } \\
\text { Skin with blubber to U of Hawaii } \\
\text { Blood serum to U of AK, Fairbanks, AK } \\
\text { Histological sample to NIST, NBSB }\end{array}$} \\
\hline
\end{tabular}

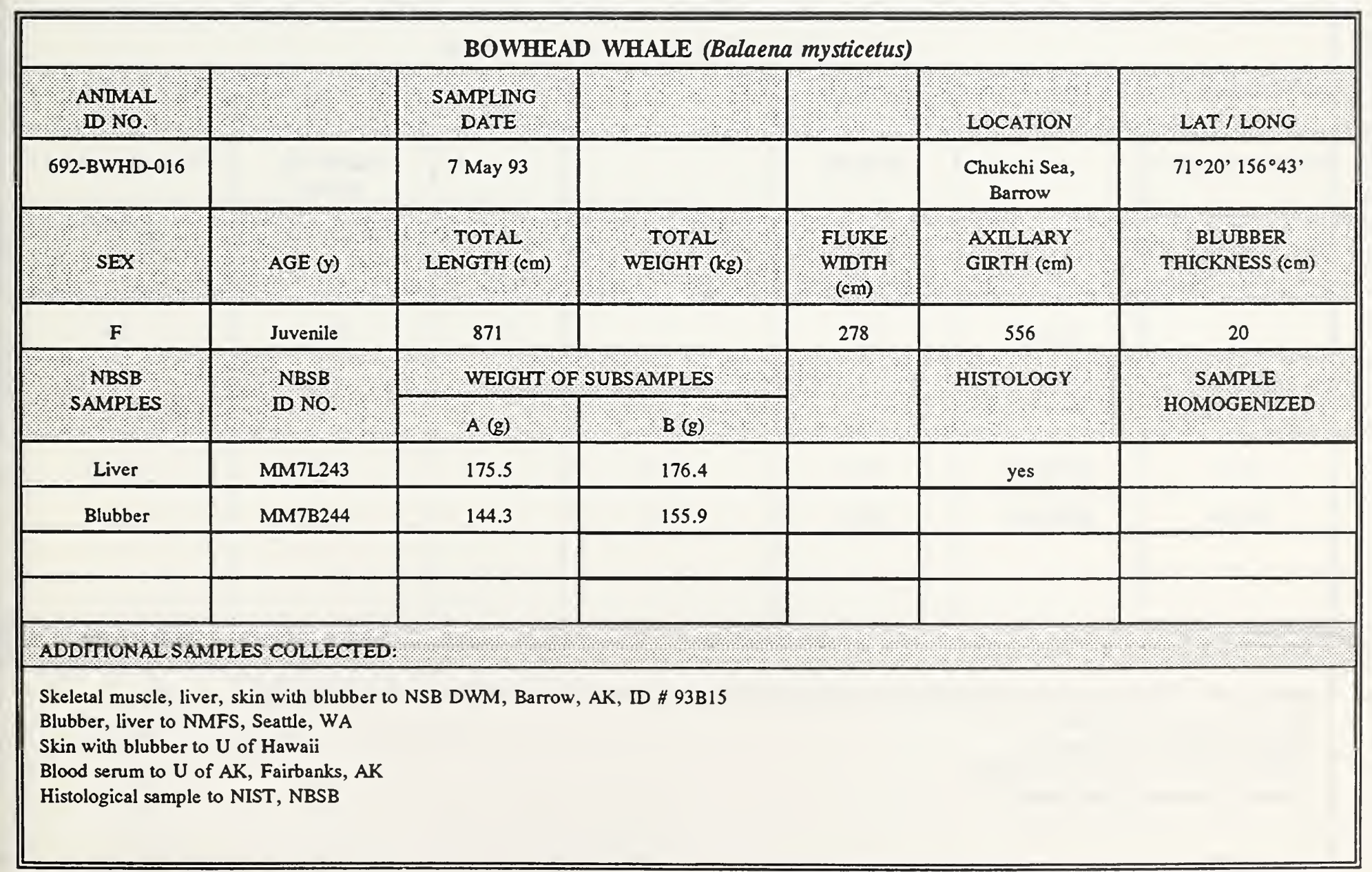




\begin{tabular}{|c|c|c|c|c|c|c|}
\hline \multicolumn{7}{|c|}{ BOWHEAD WHALE (Balaena mysticetus) } \\
\hline $\begin{array}{l}\text { ANIMAI. } \\
\text { D NO. }\end{array}$ & & SAMPLING & & & LOCATION & LAT / LONG \\
\hline 692-BWHD-017 & & 11 May 93 & & & $\begin{array}{l}\text { Chukchi Sea, } \\
\text { Barrow }\end{array}$ & $71^{\circ} 20^{\prime} 156^{\circ} 43^{\prime}$ \\
\hline SEX & $\mathrm{AGE}(y)$ & $\begin{array}{l}\text { TOTAL } \\
\text { LENGTH }(\mathrm{cm}) \text {. }\end{array}$ & $\begin{array}{l}\text { TOTAL } \\
\text { WEIGHT }(\mathrm{kg})\end{array}$ & $\begin{array}{l}\text { FLUKE. } \\
\text { wIDTH } \\
\text { (cm) }\end{array}$ & $\begin{array}{l}\text { AXILLARY } \\
\text { GRTH (cm) }\end{array}$ & THICRNESS (col) \\
\hline $\mathrm{F}$ & Juvenile & 1105 & & 304 & 572 & 18 \\
\hline NBSB & NBSB & \multicolumn{2}{|c|}{ WEIGHT OF SUBSAMPLES } & & \multirow[t]{2}{*}{ HISTOLOGY } & \multirow{2}{*}{ HOMOGENIZED } \\
\hline SAMPLES & $\mathrm{IDNO}$ & $A(g)$ & $\mathrm{B}(\mathrm{g})$ & & & \\
\hline Liver & MM7L245 & 154.8 & 156.2 & & yes & \\
\hline Blubber & MM7B246 & 140.0 & 160.0 & & & \\
\hline & & & & & & \\
\hline \multirow{2}{*}{\multicolumn{7}{|c|}{ ADDITIONAL SAMPLES COLLECTED. }} \\
\hline & & & & & & \\
\hline \multicolumn{7}{|c|}{$\begin{array}{l}\text { Skeletal muscle, liver, skin with blubber to NSB DWM, Barrow, AK, ID \# } 93 \text { B16 } \\
\text { Blubber, liver to NMFS, Seattle, WA } \\
\text { Skin with blubber to U of Hawaii } \\
\text { Blood serum to U of AK, Fairbanks, AK } \\
\text { Histological sample to NIST, NBSB }\end{array}$} \\
\hline
\end{tabular}

\begin{tabular}{|c|c|c|c|c|c|c|}
\hline \multicolumn{7}{|c|}{ BOWHEAD WHALE (Balaena mysticetus) } \\
\hline $\begin{array}{l}\text { ANIMAL: } \\
\text { ID NO. }\end{array}$ & & $\begin{array}{l}\text { SAMPLING } \\
\text { DATE }\end{array}$ & & & LOCATION & LAT / LONG \\
\hline 692-BWHD-020 & & 13 Oct 93 & & & $\begin{array}{l}\text { Chukchi Sea, } \\
\text { Barrow }\end{array}$ & $71^{\circ} 26.53^{\prime} 156^{\circ} 53.7$ \\
\hline SEX & $A G E(y)$ & $\begin{array}{l}\text {. TOTAL } \\
\text { LENGTH }(\mathrm{cm}) \text {. }\end{array}$ & $\begin{array}{l}\text { TOTAL } \\
\text { WEIGHT (kg) }\end{array}$ & $\begin{array}{l}\text { FLURE } \\
\text { WIDTH } \\
(\mathrm{cm})\end{array}$ & $\begin{array}{l}\text { AXILLARY } \\
\text { GRTH }(\mathrm{cm})\end{array}$ & THICKNESS (cm) \\
\hline $\mathbf{M}$ & Juvenile & 1361 & & & 830 & 26 \\
\hline : NBSB & \multirow{2}{*}{ NBSB: } & \multicolumn{2}{|c|}{ WEIGHT OF SUBSAMPLES } & & \multirow[t]{2}{*}{ HistoloG Y } & \multirow{2}{*}{, HOMOGENIZED } \\
\hline SAMPLES & & $A(g)$ & B (g) & & & \\
\hline Liver & MM8L259 & 163.8 & 159.3 & & yes & \\
\hline Blubber & MM8B260 & 140.0 & 140.1 & & & \\
\hline & & & & & & \\
\hline & & & & & & \\
\hline \multicolumn{7}{|c|}{ ADDITIONAL SAMPLES COLLECTED: } \\
\hline \multicolumn{7}{|c|}{$\begin{array}{l}\text { Kidney, liver, skeletal muscle, testes in } 10 \% \\
\text { Blubber, liver to NMFS, Seattle, WA } \\
\text { Skin with blubber to U of Hawaii } \\
\text { Blood serum to U of AK, Fairbanks, AK } \\
\text { Histological sample to NIST, NBSB }\end{array}$} \\
\hline
\end{tabular}




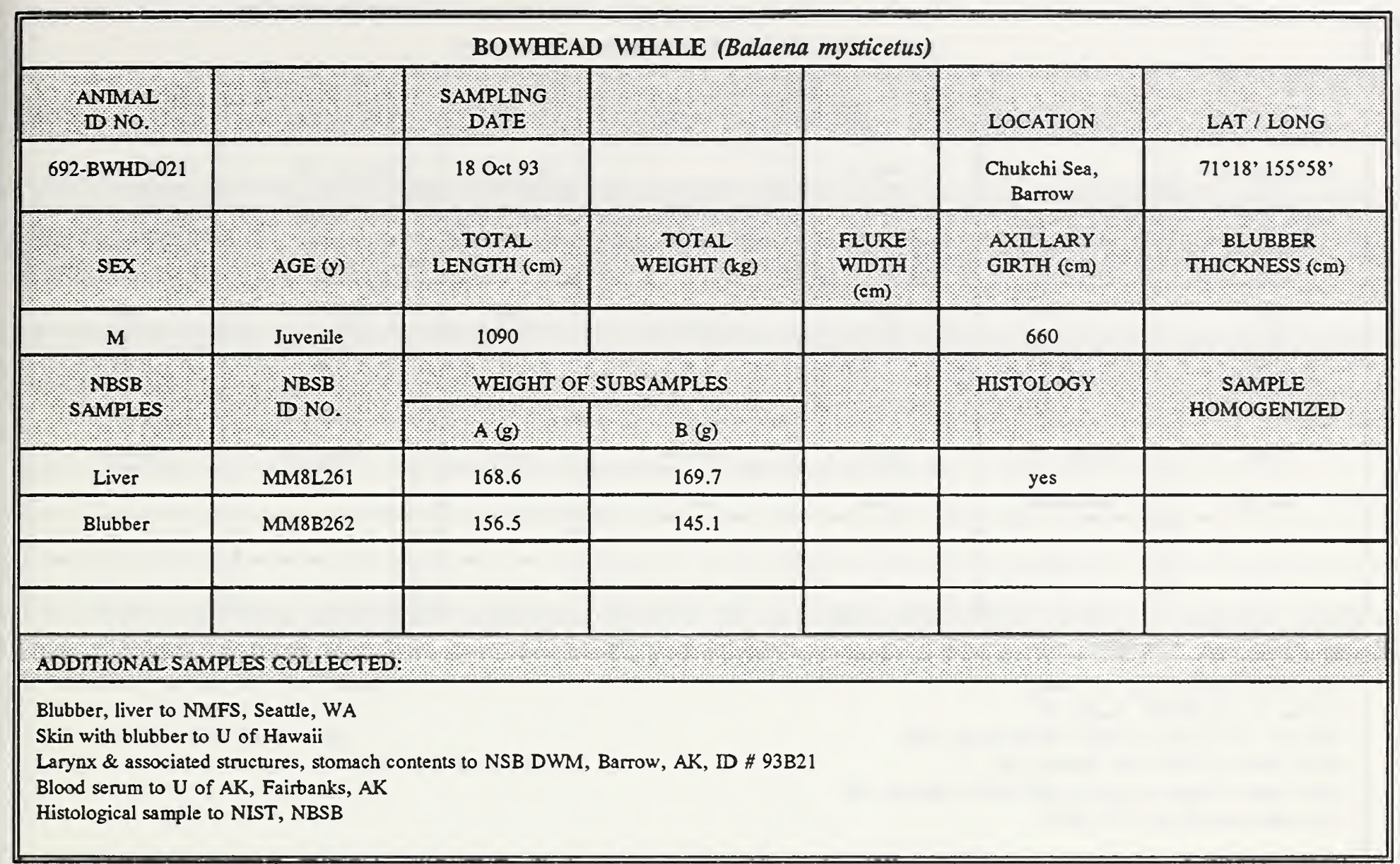

\begin{tabular}{|c|c|c|c|c|c|c|}
\hline \multicolumn{7}{|c|}{ BOWHEAD WHALE (Balaena mysticetus) } \\
\hline ANLMAI & & DATE & & & LOCATION & LAT / LONG \\
\hline 692-BWHD-022 & 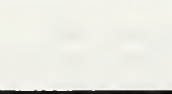 & 18 Oct 93 & & & $\begin{array}{c}\text { Chukchi Sea, } \\
\text { Barrow }\end{array}$ & $71^{\circ} 23.38^{\prime} 155^{\circ} 50.38^{\prime}$ \\
\hline SEX & AGE $(y)$ & $\begin{array}{l}\text { TOTAL } \\
\text { LENGTH }(\mathrm{cm}) \text {. }\end{array}$ & WEIGHT (kg) & $\begin{array}{l}\text { FLURE } \\
\text { WIDTH } \\
\text { (cm) }\end{array}$ & $\begin{array}{l}\text { AXILLARY } \\
\text { GIRTH }(\mathrm{cm})\end{array}$ & $\begin{array}{l}\text { BLUBBER } \\
\text { THICKNESS (cm) }\end{array}$ \\
\hline M & & 1260 & & 449 & 774 & \\
\hline \multirow{2}{*}{$\begin{array}{l}\text { NBSB } \\
\text { SAMPLES }\end{array}$} & \multirow{2}{*}{$\begin{array}{l}\text { NBSB } \\
\text { D NO. }\end{array}$} & \multicolumn{2}{|c|}{ WEIGHT OF SUBSAMPLES } & & \multirow[t]{2}{*}{ HISTOLOGY } & \multirow{2}{*}{$\begin{array}{l}\text { SAMPLE } \\
\text { HOMOGENIZED }\end{array}$} \\
\hline & & $A(g)$ & $B(g)$ & & & \\
\hline Blubber & MM8B263 & 155.6 & 159.8 & & & 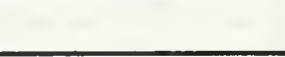 \\
\hline & & & & & & \\
\hline & & & & & & \\
\hline \multicolumn{7}{|c|}{ ADDMIONAL SAMPLES COLLECTED. } \\
\hline \multicolumn{7}{|c|}{$\begin{array}{l}\text { Stomach contents to NSB DWM, Barrow, AK, DD \#93B22 } \\
\text { Blubber to NMFS, Seattle, WA } \\
\text { Skin with blubber to U of Hawaii } \\
\text { Blood serum to U of AK, Fairbanks, AK }\end{array}$} \\
\hline
\end{tabular}




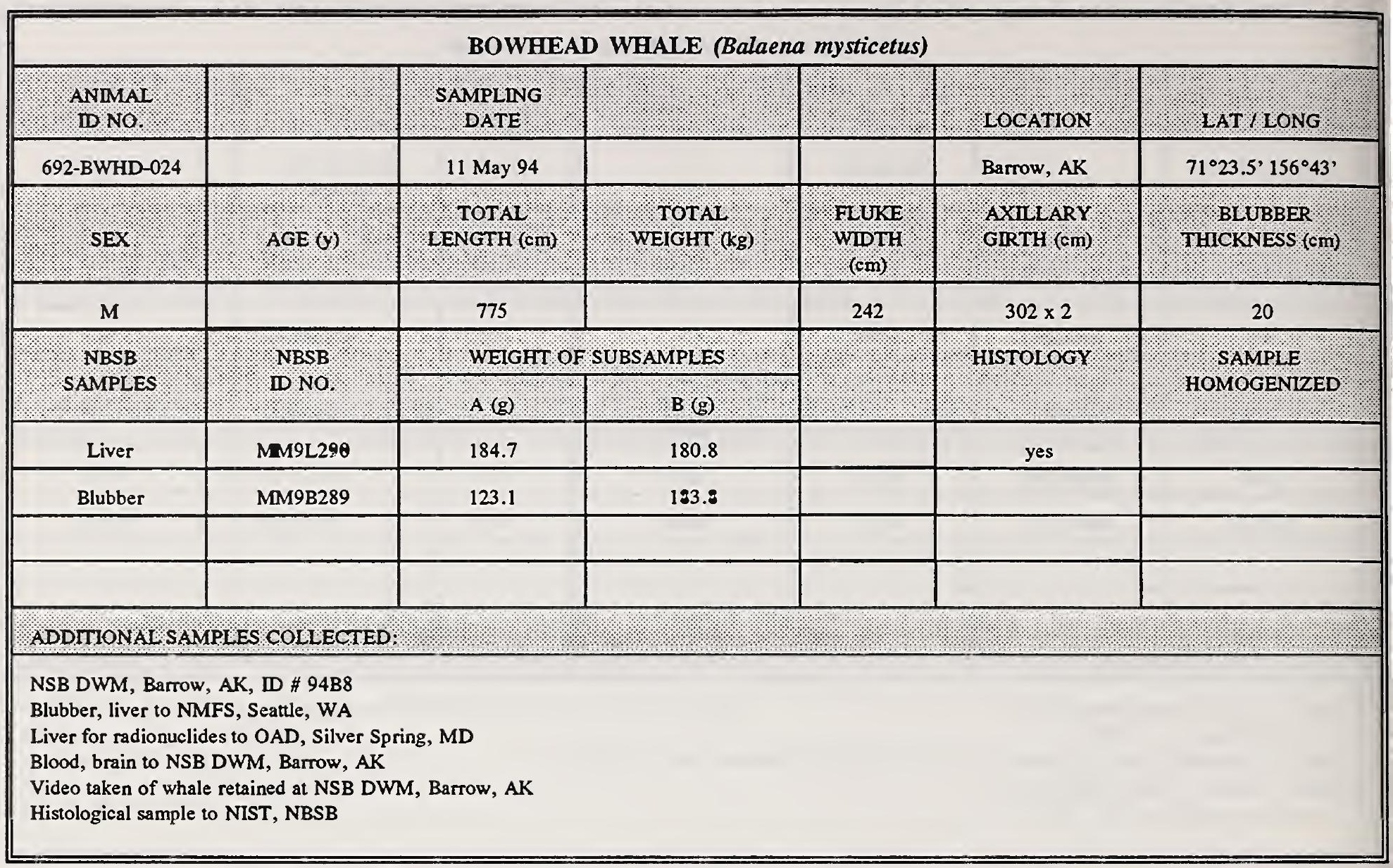

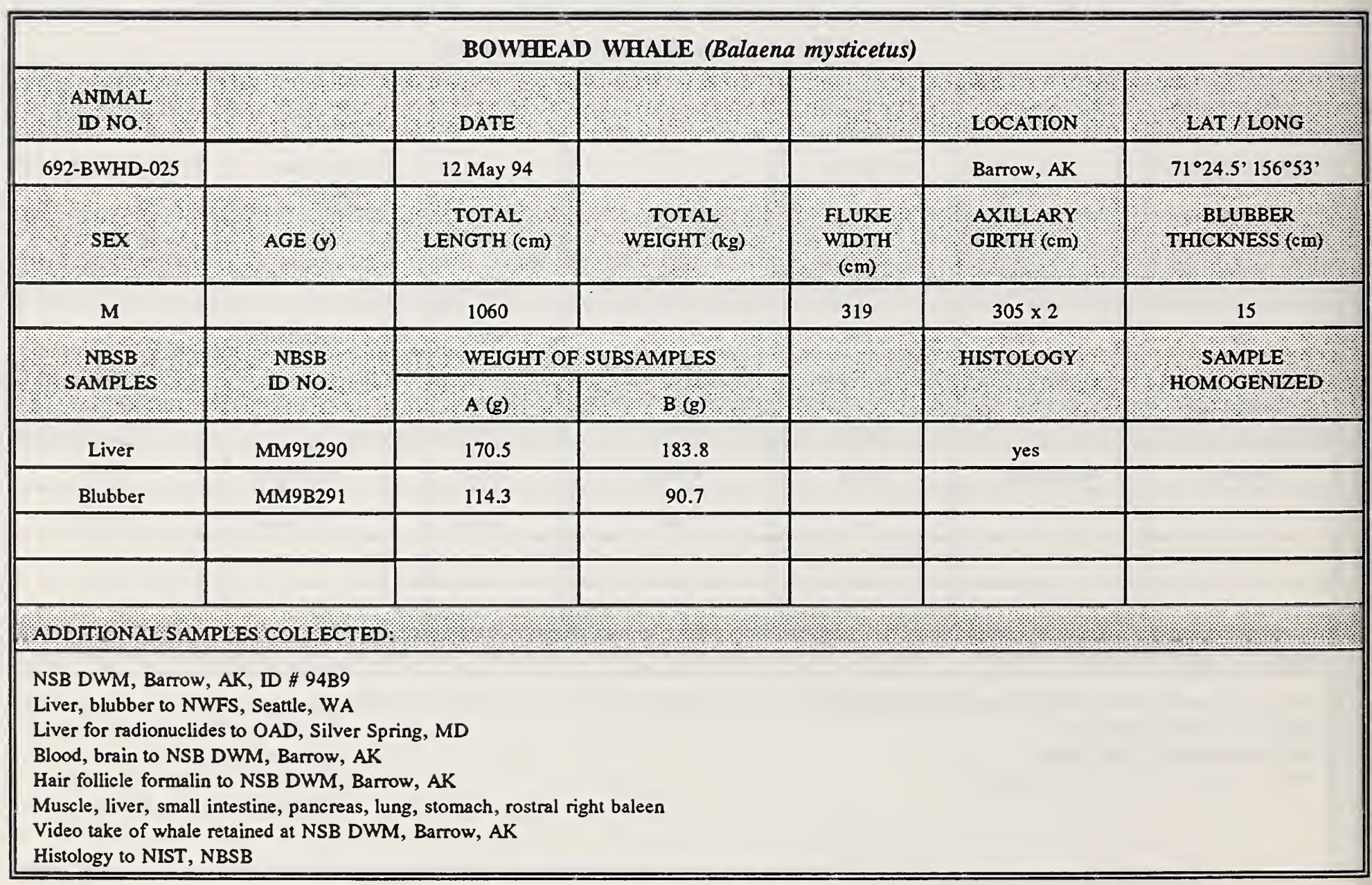




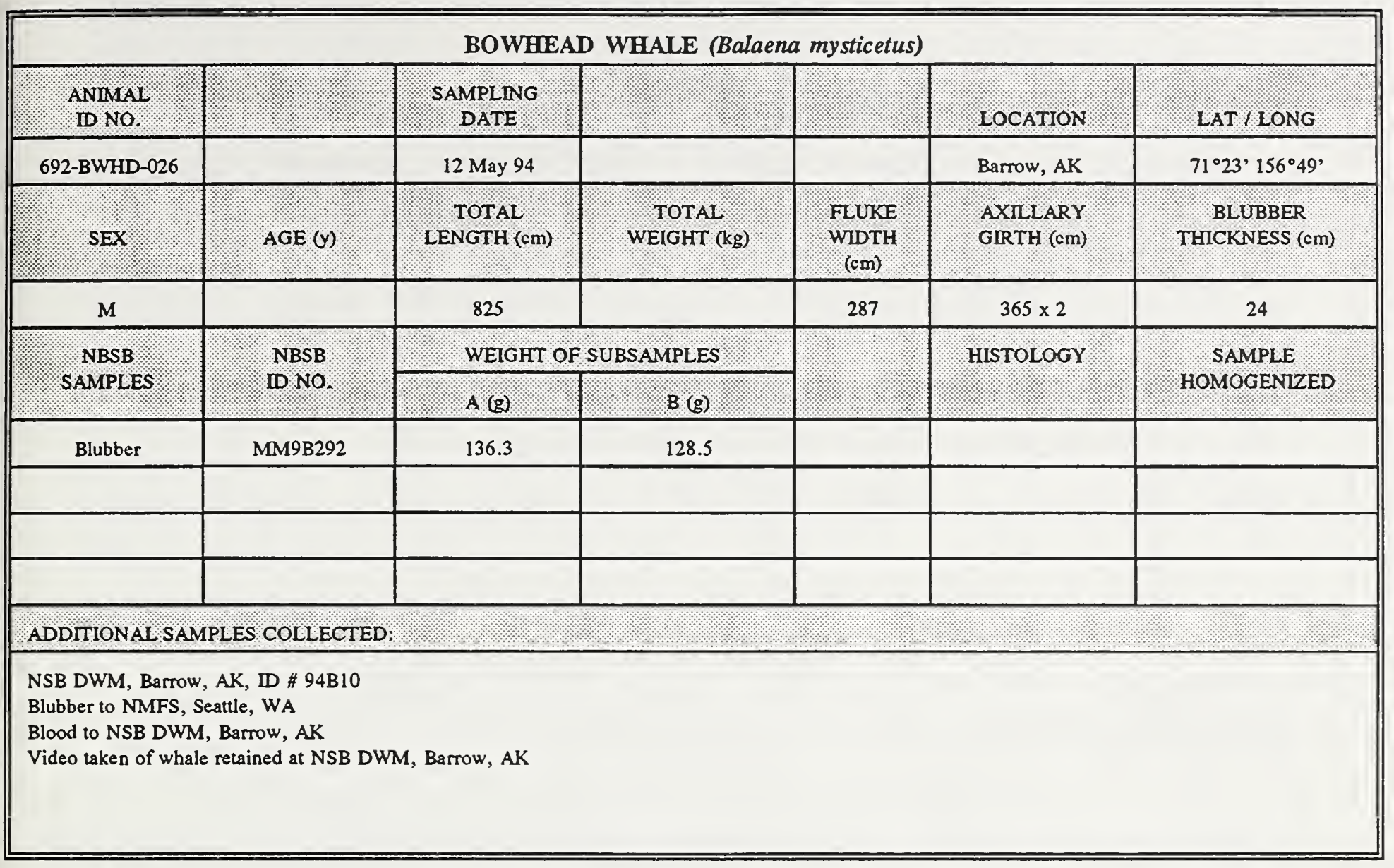

\begin{tabular}{|c|c|c|c|c|c|c|}
\hline \multicolumn{7}{|c|}{ BOWHEAD WHALE (Balaena mysticetus) } \\
\hline ANM NoI & & DATE & & & LOCATION & LAT / LONG \\
\hline 692-BWHD-027 & & 14 May 94 & & & Barrow, AK & $71^{\circ} 25^{\prime} 156^{\circ} 44^{\prime}$ \\
\hline SEX & AGE $(y)$ & LENGTH (cm) & 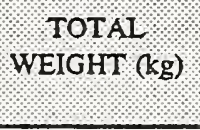 & $\begin{array}{l}\text { FLURE } \\
\text { WIDTH } \\
\text { (cm) }\end{array}$ & $\begin{array}{l}\text { AXIILARY } \\
\text { GIRTH (cm) }\end{array}$ & $\begin{array}{l}\text { BLUBBER } \\
\text { THICKNESS (cm) }\end{array}$ \\
\hline $\mathrm{M}$ & & 869 & & 287 & $272 \times 2$ & 18 \\
\hline NBSB & NBSB & \multicolumn{2}{|c|}{ WEIGHT OF SUBSAMPLES } & & \multirow[t]{2}{*}{ HistoloGY } & \multirow{2}{*}{ SAMOGENIZEE } \\
\hline & & $A(g)$ & $B(g)$ & & & \\
\hline Liver & MM9L293 & 177.6 & 175.8 & & yes & \\
\hline Blubber & MM9B294 & 123.1 & 121.4 & & & \\
\hline & & & & & & \\
\hline \multicolumn{7}{|c|}{ ADDITIONAL SAMPLES COLLECTED. } \\
\hline \multicolumn{7}{|c|}{$\begin{array}{l}\text { NSB DWM, Barrow, AK, ID \# 94B11 } \\
\text { Liver, blubber to NWFS, Seattle, WA } \\
\text { Liver for radionuclides to OAD, Silver Spring, MD } \\
\text { Histology to NIST, NBSB }\end{array}$} \\
\hline
\end{tabular}


